\title{
BRAIN SIGNAL PREDICTIONS FROM MULTI-SCALE NETWORKS USING A LINEARIZED FRAMEWORK
}

\author{
๑ Espen Hagen $^{1 *}$ \\ (1) Steinn H. Magnusson ${ }^{2}$ \\ 구 Torbjørn V. Ness ${ }^{3}$ \\ (1) Geir Halnes ${ }^{3}$ \\ (ㄴ) Pooja N. Babu ${ }^{4}$ \\ (ㄷ) Charl Linssen ${ }^{4,5}$ \\ (1) Abigail Morrison ${ }^{4,5,6}$ \\ (1) Gaute T. Einevoll ${ }^{2,3 \dagger}$

\begin{abstract}
1 Department of Data Science, Faculty of Science and Technology, Norwegian University of Life Sciences, Ås, Norway
2 Department of Physics, Faculty of Mathematics and Natural Sciences, University of Oslo, Oslo, Norway

3 Department of Physics, Faculty of Science and Technology, Norwegian University of Life Sciences, Ås, Norway

4 Simulation \& Data Lab Neuroscience, Institute for Advanced Simulation, Jülich Supercomputing Centre (JSC), Jülich Research Centre, Jülich, Germany

5 Institute of Neuroscience and Medicine (INM-6); Computational and Systems Neuroscience \& Institute for Advanced Simulation (IAS-6);

Theoretical Neuroscience \& JARA-Institute Brain Structure-Function Relationships (INM-10), Jülich Research Centre and JARA, Jülich, Germany

6 Software Engineering, Department of Computer Science 3, RWTH Aachen University, Aachen, Germany
\end{abstract}

\begin{abstract}
Simulations of neural activity at different levels of detail have become ubiquitous in modern neurosciences, aiding the interpretation of experimental data and underlying neural mechanisms at the level of cells and circuits. Extracellular electric measurements of brain signals that reflect transmembrane currents throughout the neural tissue remain commonplace. The lower frequencies $(\lesssim 300 \mathrm{~Hz}$ or so) of measured signals generally stem from synaptic activity driven by recurrent interactions among neural populations and computational models should also incorporate accurate predictions of such signals. Due to limited computational resources, large scale neuronal network models $\left(\gtrsim 10^{6}\right.$ neurons or so) often require reducing the level of biophysical detail and account mainly for times of action potentials ('spikes') or spike rates, and corresponding extracellular signal predictions have thus poorly accounted for their biophysical origin.

Here we propose a computational framework for predicting spatiotemporal filter kernels for such extracellular signals stemming from synaptic activity, accounting for the biophysics of neurons, populations and recurrent connections. Signals are obtained by convolving population spike rates by appropriate kernels for each connection pathway and summing the contributions. Our main results are that kernels derived via linearized synapse and membrane dynamics, distributions of cells, conduction delay and volume conductor model allows for accurately capturing the spatiotemporal dynamics of ground truth extracellular signals. One particular observation is that changes in the effective membrane time constants caused by persistent synapse activation must be accounted for.

The work also constitutes a major advance in computational efficacy of accurate, biophysics-based signal predictions from large scale spike and rate-based neuron network models drastically reducing signal prediction times compared to biophysically detailed network models. This work also provides insight into how experimentally recorded low-frequency extracellular signals of neuronal activity may be approximately linearly dependent on spiking activity. A new software tool LFPykernels serves as a reference implementation of the framework.
\end{abstract}

Keywords LFP · EEG · MEG · forward modeling · neuron network models $\cdot$ kernel methods

\footnotetext{
*Corresponding author: espen.hagen@nmbu.no

${ }^{\dagger}$ Corresponding author: gaute.einevoll@nmbu.no
} 


\section{Significance Statement}

Understanding the brain's function and activity in healthy and pathological states across spatial scales and times spanning entire lives is one of humanity's great undertakings. In experimental and clinical work probing its activity using a variety of electric and magnetic measurement devices are routinely performed, however interpreting the signals measured extracellularly remains arduous due to multiple factors, like the large number of neurons contributing to the signals and complex interactions occurring in recurrently connected neuronal circuits. To understand how neurons give rise to such signals, mechanistic modeling combined with forward models derived using volume conductor theory has proven to be successful, but this approach currently does not scale to the systems level (encompassing millions of neurons or more) where simplified or abstract neuron representations typically are used. Motivated by experimental findings implying approximately linear relationships between times of neuronal action potentials and extracellular population signals, we provide a biophysics based method for computing causal filters relating spikes and extracellular signals that can be applied with spike times or rates of large scale neuronal network models for predictions of population signals without relying on ad hoc approximations.

\section{Introduction}

Extracellular electric recordings of neuronal activity, either by embedding sharp electrodes in neural tissue (Hubel 1957) or by placing electrodes on top of cortex (Berger 1929) or on the scalp (electroencephalography - EEG, Nunez and Srinivasan 2006), have a long history in the experimental and clinical neurosciences. The same applies to magnetic recordings outside of the head (magnetoencephalography - MEG, Hämäläinen et al. 1993). However, the link between the measured brain signals and the underlying neuronal activity remains poorly understood due to the inherent ill-posed inverse problem: The number of contributing sources is large compared to the limited number of discrete locations in- and outside of the brain tissue where one can measure. However, the forward problem is well posed, given the transmembrane currents in all neurons setting up the activity. Different electric and magnetic signals can be computed by means of so-called volume conductor (VC) theory mapping source currents to each signal type, thus models accounting for the biophysical properties of neurons and networks thereof can now be used to study the link between activity and measurements (Einevoll et al. 2013; Einevoll et al. 2019).

Dynamics of biophysically detailed neurons and synaptically coupled networks thereof are typically modeled by solving sets of coupled, linear and non-linear ordinary or partial differential equations describing the dynamics of the neuronal membranes, ion channel conductances, synapses and so forth (see e.g., Schutter 2010). Multicompartment (MC) models have for decades been the go-to tool for geometrically detailed conductance-based neuron models as tailored software solvers are readily available such as NEURON (Carnevale and Hines 2006) and Arbor (Akar et al. 2019). For the purpose of computing extracellular electric and magnetic signals, transmembrane currents from the MC neuron simulation is then combined with the appropriate forward model derived using linear volume conductor theory, as incorporated in software interfacing the neural simulator like LFPy (Lindén et al. 2014; Hagen et al. 2018), NetPyNe (Dura-Bernal et al. 2019) and BMTK (Dai et al. 2020). For brain tissues, a linear relationship between transmembrane currents and extracellular electric potentials as well as magnetic fields appears well established (Nicholson and Freeman 1975; Nunez and Srinivasan 2006; Logothetis et al. 2007; Miceli et al. 2017).

Illustrated in Figure 1, neuronal network models may account for different levels of detail, ranging from biophysically detailed MC neuron networks (top level), simplified spiking point-neuron networks (middle level) and population type models accounting for population-averaged activity (bottom level). The different levels may at times be bridged with appropriate mapping of parameters. Illustrated in the figure, MC models may be directly combined with VC theory for extracellular signal predictions as these model account for the spatiotemporal distribution of transmembrane currents, while the less detailed model descriptions, in particular point-neuron networks and mean-field type population models, do not. Thus in order to relate their activity in terms of spike times or spike rates of the different populations to extracellular signals additional steps are required, here illustrated by some 'black box' model taking spikes or equivalent spike rates of each population as input while outputting approximated extracellular signals. Here we shall approach this black box problem by models that account for key properties of the biophysically detailed network models (mainly cell model membrane dynamics, spatial distributions of cells and synapses, network connectivity, temporal synapse dynamics), properties which could also be constrained by experimental data. These particular approximate models considered (in particular the 'hybrid scheme' and 'kernel based' models) are linear, and applicable to predict the low-frequency content $(\lesssim 300 \mathrm{~Hz}$ or so) of the signals usually associated with population activity and network interactions.

Thus, throughout this paper we in part consider observations and assumptions of near linear relationships between times of neuronal action potentials (APs) as well as their extracellularly recorded correlates ('spikes'), and low-frequency 


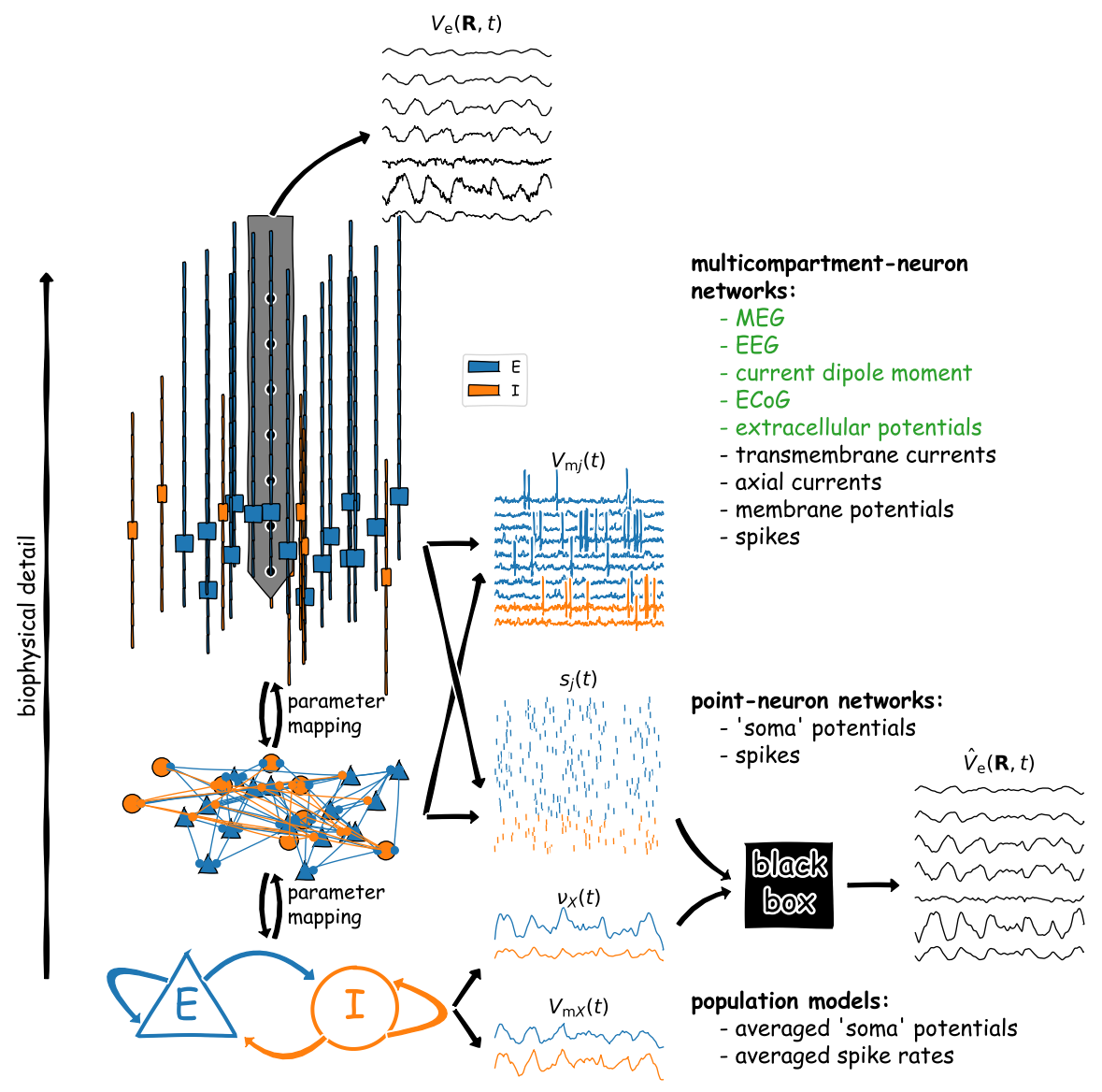

Figure 1: Biophysical levels detail for neuronal network models and signal predictions. Biophysically detailed MC neuron network models at the microscopic scale allow for simulating synaptic connectivity, membrane voltage and current dynamics across the entire cell, APs, spike trains and extracellular signals (e.g., the extracellular potential $\left.V_{\mathrm{e}}(\mathbf{R}, t)\right)$ using forward models derived via VC theory. Neither less detailed spiking point-neuron network models nor continuous population type models (neural mass models, mean field models, neural field models) towards the mesoscopic scale facilitate extracellular signal predictions. Such model descriptions require some intermediate model translating spike events or spike rates into representative extracellular signal approximation $\left(\hat{V}_{\mathrm{e}}(\mathbf{R}, t)\right)$, here illustrated by the black box.

parts (below a few hundred hertz) of extracellularly recorded population signals like local field potential (LFP), EEG and MEG signals (Einevoll et al. 2007; Rasch et al. 2009; Hall et al. 2014; Ahmadi et al. 2021).

For synaptically coupled neuronal networks, one may consider two main direct neuronal contributors to extracellular populations signals. The first is due to presynaptic neurons generating APs observed as extracellular spikes nearby each active neuron in recordings using invasive microelectrodes. The second is due to evoked synaptic currents and associated membrane currents throughout postsynaptic populations following presynaptic APs. AP durations are on the order of milliseconds, APs occur with relative sparsity (low spike rates) and irregularity in single-neuron spike trains (see e.g., Mochizuki et al. 2016), observed pairwise spike train correlations are weak (Ecker et al. 2010; Renart et al. 2010), and extracellular spike amplitudes decay quickly with distance (see e.g., Pettersen and Einevoll 2008; Hagen et al. 2015). Extracellular spikes also carry more power toward higher frequencies (Pettersen and Einevoll 2008). The latter synaptic contributions can be small in amplitude per pair of pre- and postsynaptic neurons relative to currents related to each presynaptic AP itself, but each neuron typically targets many neurons via hundred or even thousands of synapses, and recurrent interactions may affect times of subsequent activations across large neural populations. The dynamics of synapse currents are also relatively slow and can thus be assumed to shape extracellular signals around lower frequencies than presynaptic contributions. Thus, we shall assume that mainly synaptic activity govern the 
low-frequency content of extracellular signals, in part contributed by a boosting effect on the compound signals by even weak pairwise spike train correlations (Hagen et al. 2016).

Dynamics of neuronal activity are typically nonlinear, one prime example being the model for APs by Hodgkin and Huxley 1952 which also provided a mathematical formalism which remains commonly used to describe different ion-channel dynamics (e.g., Hay et al. 2011). Extracellularly recorded postsynaptic responses following presynaptic AP events can not initially be assumed to be linear, as synaptic currents following activation are not linearly dependent on the synaptic conductance due to membrane potential changes. Furthermore, there may be active (voltage- and calcium-dependent) ion channels present across dendrites resulting in non-linear integration even below AP threshold, and contributions by different activations of multiple synapses may not sum linearly (Larkum et al. 2001). Synaptic activity may also result in dendritic $\mathrm{Ca}^{2+}$ - and NMDA spikes (Major et al. 2013).

Thus to explain experimental data implying approximately linear relationships between times of presynaptic spikes and different electric signals, the direct signal contribution by both pre- and postsynaptic activity must sum approximately linearly. Furthermore, for synaptic currents across postsynaptic populations the different contributions by non-linearities in terms of nonlinear synapse and membrane dynamics must be negligible or well explained by linear components around typical working points (e.g., average membrane potentials and spike rates). Still, a number of computational studies assume linearity between presynaptic spike events and corresponding times of synaptic activations and resulting extracellularly recorded signals (Tomsett et al. 2014; Mazzoni et al. 2015; Głąbska et al. 2016; Hagen et al. 2016; Senk et al. 2018b; Senk et al. 2018a; Teleńczuk et al. 2020a; Skaar et al. 2020). Others assume linearity between transmembrane input current input and extracellular potentials (Lindén et al. 2010), in part justified by model work wherein dynamics of active ion channels are approximated by linear dynamics (Ness et al. 2016; Ness et al. 2018). Analysis of experimental recordings by Haider et al. 2016 also show synaptic currents and the LFP to be strongly coupled using a linear regression model.

Henceforth, we shall examine the validity of models that either explicitly or implicitly assume linear relationships between neuronal spiking and extracellular signals. We will do so by comparing the extracellular signals that these models predict with corresponding predictions obtained with biophysically detailed MC neuron networks. Hereby we test the following approaches (hypotheses): (1) Linearized model setups can accurately capture the spatiotemporal features of ground truth extracellular potential and current dipole moment computed from recurrent networks of inherently non-linear constituents. For this testing, we first apply a hybrid prediction scheme (Hagen et al. 2016) where morphologies, synaptic placements and activation times of the network is preserved allowing for computing extracellular signals but using linearized neuron and synapse dynamics. In this scheme network activity in terms of spikes is simulated independently. The scheme explicitly accounts for every postsynaptic neuron in the network. (2) If the linear hybrid scheme implementation accurately captures the ground truth signals, we test whether or not output extracellular signal predictions can be well captured as a linear and time-invariant causal system, taking population spike rates as input filtered by suitable spatiotemporal causal filters. These sets of filters, or 'kernels' represent postsynaptic spike-signal impulse responses averaged over pairs of pre- and postsynaptic populations, and are initially computed via the hybrid scheme. This assumes that the detailed cell-to-cell connectivity can be ignored. (3) Knowledge of the underlying distributions of cells and synapses, conduction delays, linearized cell and synapse dynamics and corresponding population spike rates is sufficient information to predict these spatiotemporal causal kernels accurately.

As illustrated in Figure 1, the hypothesized kernel-based approach substituting the 'black box' model can be applied with recurrent neuronal network descriptions using much-simplified neuron representations, like leaky integrate-andfire (LIF) point neurons, variants thereof as well as few-compartment neuron models, as the main determinant for the extracellular signals is presynaptic spikes, or spike rates. As also point-neuron networks may accurately mimic experimentally observed spiking activity as well as corresponding MC neuron networks (see e.g., Billeh et al. 2020), the computationally costly MC simulations may only be required in order to compute the appropriate sets of kernels, thus reducing compute resource demands by orders of magnitude. This may for instance open for efficient forward-model based extracellular signal predictions from large scale point-neuron network models encompassing multiple brain areas (Schmidt et al. 2018) or models incorporating realistic cell densities within an area (Senk et al. 2018b; Billeh et al. 2020). The kernel methodology would also be immediately useful with rate-based frameworks, as also population spike rates of spiking network models may be accurately captured in corresponding population rate models (see e.g., Gerstner 2014; Cain et al. 2016; Dumont et al. 2017; Schwalger et al. 2017).

This study is organized as follows: In Methods we first detail a generic biophysically detailed MC neuron network that is used for ground truth signal predictions, and different network configurations. Then we detail a proposed hybrid methodology that allows for separation between simulations of network activity ('spikes') and extracellular signals, and the the derivation of linearized signal predictions, including our proposed methodology for fast, accurate and deterministic predictions of kernels. In Results we investigate the properties of neuron models in active and linearized versions, recurrent MC neuron networks and compare different linear approximations to the corresponding ground truth 
signals. Then, we showcase the kernel-based methodology to network spiking activity of a recurrent network of leaky integrate-and-fire neurons. In Discussion we discuss the implications of this work and possible future steps.

\section{Methods}

\subsection{Multicompartment neuron network description}

We first define the properties of a generic recurrently connected network of $\mathrm{MC}$ neurons used for ground truth signal generation and later signal approximations. For compactness, we choose a symbolic notation similar to Hagen et al. 2016 wherever possible and provide the model details as a generic 'recipe'. Their particular values are summarized in Section 2.1.1 and Table 1, 2 and 3. Then, in general terms we:

1. Let $X \in\{\ldots\}$ and $Y \subseteq X$ denote pre- and postsynaptic populations, respectively. Each population corresponds to separate classes of neurons (derived from anatomy, electrical properties, gene expression, phenomenology, etc.). We let populations $Y$ be a local subset to allow for remote neuronal populations in $X$. (Thus $X$ may include remote populations, point processes, external stimuli and similar, which we will assume give approximately zero direct contributions to the local signals predicted by the full recurrent network model).

2. Let lists $N_{X}$ and $N_{Y}$ denote the sizes of populations $X$ and $Y$.

3. Let $u \in\left\{1, \ldots, N \mid N \in N_{X}\right\}$ and $v \in\left\{1, \ldots, N \mid N \in N_{Y}\right\}$ denote pre- and postsynaptic neuron indices, respectively.

4. Let $\mathbf{r}_{v} \sim \widetilde{\mathbf{r}}_{Y}$ denote a discretely sampled somatic location of neuron $v$, where $\widetilde{\mathbf{r}}_{Y}$ describes the probability density function of somatic locations of population $Y$ in space.

5. Let $K_{Y X}$ denote the total number of pairwise connections between presynaptic (source) population $X$ and postsynaptic (target) population $Y$. Assuming a random connectivity with binomial in- and out-degree distributions the corresponding connection probability is then $C_{Y X}=1-\left(1-1 / N_{Y} N_{X}\right)^{K_{Y X}}$ (Potjans and Diesmann 2014). $K_{Y X} \approx C_{Y X} N_{Y} N_{X}$ for small connection probabilities. The subscript $Y X$ notation is used throughout this paper to emphasize that these parameters are connection specific.

6. Let $k_{v u} \sim \widetilde{k}_{Y X}$ denote the number of synapses (multapses) per connection if a connection exist between neurons $u$ and $v . \widetilde{k}_{Y X}$ here describes a discrete distribution from which integer numbers greater than 0 are drawn.

7. Let probabilities of synapse placement onto postsynaptic compartments indexed by $m$ be proportional to the product $L_{Y X}\left(z_{m}\right) A_{m}$, where $L_{Y X}(z)$ is a depth-dependent function evaluated at the midpoint of each compartment with surface area $A_{m}$. Compartments are indexed by $m$. Synapse placements are drawn randomly $k_{v u}$ times for each pair of connected neurons.

8. Let the current for each synapse following activation at time $t$ be described by

$$
I_{\mathrm{syn} Y X}(t)=\bar{G}_{\mathrm{syn} Y X} f_{Y X}(t)\left(V_{\mathrm{m}}(t)-E_{X}\right),
$$

where $\bar{G}_{\mathrm{syn} Y X}$ denotes the maximum synaptic conductance and $f_{Y X}(t) \in[0,1]$ the temporal kinetics of the synapse. $V_{\mathrm{m}}(t)$ denotes the postsynaptic membrane potential and $E_{X}$ denotes the reversal potential of the synapse which is determined by the presynaptic cell type (i.e., excitatory or inhibitory). For simplicity we will assume that $\bar{G}_{\mathrm{syn} Y X}$ is independent of position. We will also assume that $\bar{G}_{\mathrm{syn} Y X}$ is static, that is, there are no synaptic plasticity rules or stochastic processes in place. Individual weights are, however, drawn from a distribution described by a probability density function $\widetilde{G}_{\mathrm{syn} Y X}$.

9. Let the conduction delays resulting from presynaptic action potential generation time to activation time of the synapse be greater than zero and randomly drawn from some function as $\Delta_{v u} \sim \widetilde{\Delta}_{Y X}(t)$. For simplicity, we let conduction delays be independent of cell location and geometry.

10. Let the sequence of spike times $s_{u}(t)=\sum_{k} \delta_{t_{k}}=\sum_{k} \delta\left(t-t_{k}\right)$ of each presynaptic neuron $u$ in each population $X$ is recorded throughout the entire simulation duration $\left[0, t_{\mathrm{sim}}\right\rangle$. We may choose to relax this requirement if a population in $X$ represents an external population feeding persistent, uncorrelated events with spectrally 'flat' spiking statistics (e.g., fixed-rate Poisson point processes) into the recurrently connected network. We here (and for the remainder of this study) use the compact notation $\delta_{t_{k}}=\delta\left(t-t_{k}\right)$ to denote Dirac delta functions centered around the time $t_{k}$.

11. Let the weighted, directed graph representing edges (synaptic connections) between nodes (neurons) for every pair of pre- and postsynaptic populations be stored for purpose of validating the 'hybrid scheme' simulations 
described in Section 2.2. This storage requirement may also be relaxed if the total number of synapses $k=\sum_{u \in X} \sum_{u \in Y} k_{u v}$ is large enough to make storage infeasible or one could recreate the full connectivity graph procedurally (at least statistically). Graph weights represent maximum synaptic conductances. The graph also include the synaptic locations on the postsynaptic neurons, and we will hereby let compartment index $m$ equate this location.

12. Let each postsynaptic neuron $v$ in any population in $Y$ be modeled using the 'standard' MC neuron formalism such that their transmembrane currents $\left[I_{\mathrm{m}}^{\langle v\rangle}\left(\mathbf{r}_{m}, t\right)\right]$ per compartment indexed by $m$ can be computed. $\mathbf{r}_{m}$ denotes their midpoint coordinates.

13. Let extracellular signal contributions in different spatial locations (or axes in terms of current dipole moment) be computed and summed up as $\sum_{Y} \sum_{v=1}^{N_{Y}} \mathcal{F}^{\langle v\rangle}\left[I_{\mathrm{m}}^{\langle v\rangle}\left(\mathbf{r}_{m}, t\right)\right]$. The matrix $\mathcal{F}^{\langle v\rangle}$ here denotes a linear mapping of transmembrane currents of cell $v$ to a linearly dependent extracellular signal. The different forward models considered in this study are detailed in Section 2.4.

\subsubsection{Reference network of simplified ball-and-sticks neurons}

The two-population, recurrent MC neuron network model constructed for this study, fully specified by the bullets in Section 2.1 and parameter values listed in Table 1,2 and 3, is kept intentionally simple for clarity of results. One main simplification is stylized neuron models with only a subset of ion channels distributed onto soma and dendritic compartments. The 'E' cell is represents a phenomenological excitatory unit, while the 'I' cell represents a phenomenological inhibitory unit. Both share the same subset of passive and active ion channels taken from a biophysically detailed cell model (Hay et al. 2011), important for action potential generation (transient sodium, $\mathrm{Na}_{t}$; fast, non-activating potassium, $\mathrm{SK}_{\mathrm{v} 1.3}$ ) and sub-threshold dynamics (non-specific cation current, $\mathrm{I}_{h}$ ). Please refer to Hay et al. 2011 for details on these ion-channel dynamics. Network parameters were tuned by a combination of hand tuning and parameter value scans, aiming to generate population spiking activity that is asynchronous and irregular (AI) (Brunel 2000) and with biologically plausible spontaneous spike rates (approximately 2.5 spikes s ${ }^{-1}$ for the ' $\mathrm{E}$ ' population; 5 spikes s $^{-1}$ for the 'I' population).

\subsubsection{Ball-and-sticks neuron networks with perturbed synaptic conductances}

A part of this study is devoted to the effect of perturbed network parameters in different network instances on our proposed methodology. For this we incorporated a global synaptic weight scaling factor $J \in\{1 / 4,1 / 2,1,2,4\}$ that is multiplied with the maximum synaptic conductances $\bar{G}_{\mathrm{syn} Y X}$ (and parameters derived from it). A factor $J=1$ corresponds to our unperturbed reference network.

\subsubsection{Network of biophysically detailed neuron models}

As an additional test of the methodology developed around the above description of a recurrent network of simplified ball-and-sticks MC neuron models, we replace the 'excitatory' (E) cell type by a biophysically detailed model of a thick-tufted layer 5b pyramidal cell of rat somatosensory cortex described in detail in Hay et al. 2011. Here, we use model parameter values shown to produce acceptable BAC firing and perisomatic step current firing as summarized in Hay et al. 2011, Table 3. Each individual cell instance in the modified network model is rotated by $4.729 \mathrm{rad}$ and $-3.166 \mathrm{rad}$ around the horizontal $x-$ and $y$-axes, respectively, in order to first align the apical dendrite with the vertical $z$-axis, before applying a random rotation around the $z$-axis. By increasing the number of extrinsic synapses distributed on each neuron to $k_{\mathrm{ext}}^{E}=920$, the typical population firing rates and network state is well preserved when compared to the reference network. All other parameters remain as defined in Table 1-3.

\subsubsection{Network of leaky integrate-and-fire (LIF) point-neuron models}

As a proof of principle that the 'kernel method' (see Section 2.3) may be utilized for 'live' extracellular signal predictions with spiking point-neuron network models or other types of networks with abstract neuron representations, we fit connectivity parameters of a phenomenological two-population network of leaky integrate-and-fire (LIF) point neuron network to mimic the spiking activity of the original MC network model. After initial hand tuning of the network parameters into a reasonable state of activity resembling the reference network's state, we subsequently used the multi-objective optimization NSGA-II non-dominated sorting genetic algorithm (Deb et al. 2002) in order to fit key network connectivity parameters, namely synaptic weights $\bar{I}_{\text {syn } Y X}$, membrane capacitance of neurons in each population $C_{\mathrm{m} X}$, weight of extrinsic synapses $\bar{I}_{\text {syn }}^{\mathrm{ext}}$ and mean value of the conduction delay distributions $\left\langle\widetilde{\Delta}_{Y X}\right\rangle$. The full network and neuron description is given in Table 4, including best fit parameters and parameter value boundaries used for the fitting procedure. 
Table 1: MC neuron and recurrent network parameters (continued in Table 2). The leak, fast inactivating $\mathrm{Na}^{+}\left(\mathrm{Na}_{\mathrm{t}}\right)$, fast, non-inactivating $\mathrm{K}^{+}\left(\mathrm{Kv}_{3.1}\right)$ channel and non-specific cation current $\left(\mathrm{I}_{h}\right)$ dynamics are those given in detail in Hay et al. 2011. Capped and discrete distributions are normalized such that the integral or sum over all values equals 1.

\begin{tabular}{|c|c|c|}
\hline Symbol & Value/definition & Description \\
\hline$X$ & $\{$ Excitatory (E), inhibitory (I) $\}$ & Presynaptic cell types/populations \\
\hline$Y$ & $X$ & Postsynaptic cell types/populations \\
\hline$N_{Y} \in\left\{N_{\mathrm{E}}, N_{\mathrm{I}}\right\}$ & $\{8192,1024\}$ & Population sizes \\
\hline$r$ & $\sqrt{x^{2}+y^{2}}$ & Radius around vertical $z$-axis \\
\hline $\mathcal{N}(\mu, \sigma)(x)$ & $\frac{1}{\sqrt{2 \pi \sigma^{2}}} \exp ^{-\frac{(x-\mu)^{2}}{2 \sigma^{2}}}$ & Gaussian distribution \\
\hline$\widetilde{\mathbf{r}}_{X}$ & $\mathcal{N}(0,75 \mu \mathrm{m})(z) * r^{2}$ for $r \in[0,150 \mu \mathrm{m}]$ & Cell body probability density function \\
\hline$C_{Y X}$ & 0.05 for all $Y$ and $X$ & $\begin{array}{l}\text { Pairwise connection probability } \\
\text { (Pairwise Bernoulli; no autapses) }\end{array}$ \\
\hline$\widetilde{k}_{Y X}$ & $\left\{\begin{array}{l}\mathcal{N}(2,0.5)\left(x_{i}\right), x_{i} \in\{1,2, \ldots, 20\}, \text { for } X=\mathrm{E} \\
\mathcal{N}(5,1)\left(x_{i}\right), x_{i} \in\{1,2, \ldots, 20\}, \text { for } X=\mathrm{I}\end{array}\right.$ & Multapse probability mass function \\
\hline $\mathcal{S}$ & $\{$ soma, apic, basal $\}$ & Morphology sections \\
\hline$L_{\mathcal{S}}$ & $\left\{\begin{array}{l}\{30,1000,200\} \mu \mathrm{m} \text { for } X=\mathrm{E} \\
\{30.200,200\} \text { um for } X=\mathrm{I}\end{array}\right.$ & Section length \\
\hline$d_{\mathcal{S}}$ & $\left\{\begin{array}{l}\{30,3,2\} \mu \mathrm{m} \text { for } X=\mathrm{E} \\
\{15,2,2\} \mu \mathrm{m} \text { for } X=\mathrm{I}\end{array}\right.$ & Section diameter \\
\hline$n_{\text {seg }}^{\mathcal{S}}$ & $\left\{\begin{array}{l}\{1,21,5\} \text { for } X=\mathrm{E} \\
\{1,5,5\} \text { for } X=\mathrm{I}\end{array}\right.$ & \# of segments per section \\
\hline $\begin{array}{c}c_{\mathrm{m}} \\
R_{\mathrm{a}} \\
g_{L}^{\mathcal{S}} \\
E_{\mathrm{L}} \\
\bar{g}_{\mathrm{Na}_{\mathrm{t}}}^{\mathcal{S}} \\
E_{\mathrm{Na}} \\
\bar{g}_{\mathrm{Kv}_{3.1}}^{\mathcal{S}} \\
E_{\mathrm{K}}^{\mathcal{S}} \\
\bar{g}_{\mathrm{Ih}}^{\mathcal{S}}\end{array}$ & $\begin{array}{c}1 \mu \mathrm{F} \mathrm{cm} \mathrm{cm}^{-2} \\
100 \Omega \mathrm{cm} \\
\{0.0000338,0.0000589,0.0000589\} \mathrm{S} \mathrm{cm}^{-2} \\
-90 \mathrm{mV} \\
\{2.04,0.0213,0.0213\} \mathrm{S} \mathrm{cm}^{-2} \\
50 \mathrm{mV} \\
\{0.693,0.000261,0.000261\} \mathrm{S} \mathrm{cm}^{-2} \\
-85 \mathrm{mV} \\
\{0.0002,0.002,0.002\} \mathrm{S} \mathrm{cm}^{-2}\end{array}$ & $\begin{array}{c}\text { Membrane capacitance } \\
\text { Axial resistivity } \\
\text { Passive leak conductance } \\
\text { Passive leak reversal potential } \\
\mathrm{Na}_{\mathrm{t}} \text { conductance } \\
\mathrm{Na}^{+} \text {reversal potential } \\
\mathrm{Kv}_{3.1} \text { conductance } \\
\mathrm{K}^{+} \text {reversal potential } \\
\mathrm{I}_{\mathrm{h}} \text {-current conductance }\end{array}$ \\
\hline
\end{tabular}

For the fitting, we used the implementation of the NSGA-II class pymoo.algorithms.nsga2.NSGA2 provided by the pymoo Python package (Blank and Deb 2020). The objective functions to be minimized using the pymoo.optimize.minimize method we defined as

$$
\begin{aligned}
& F_{1}=\sqrt{\left(\left\langle\nu_{E}\right\rangle-\left\langle\nu_{E}^{\mathrm{LIF}}\right\rangle\right)^{2}} \\
& F_{2}=\sqrt{\left(\left\langle\nu_{I}\right\rangle-\left\langle\nu_{I}^{\mathrm{LIF}}\right\rangle\right)^{2}} \\
& F_{3}=\sum_{f \leq 200 \mathrm{~Hz}} \sqrt{\left(S_{\left\langle\nu_{E}\right\rangle\left\langle\nu_{E}\right\rangle}(f)-S_{\left\langle\nu_{E}^{\mathrm{LIF}}\right\rangle\left\langle\nu_{E}^{\mathrm{LIF} F}\right.}(f)\right)^{2}} \\
& F_{4}=\sum_{f \leq 200 \mathrm{~Hz}} \sqrt{\left(S_{\left\langle\nu_{I}\right\rangle\left\langle\nu_{I}\right\rangle}(f)-S_{\left\langle\nu_{I}^{\mathrm{LIF}}\right\rangle\left\langle\nu_{I}^{\mathrm{LIF}}\right\rangle}(f)\right)^{2}} .
\end{aligned}
$$

Here, $\left\langle\nu_{X}\right\rangle$ and $\left\langle\nu_{X}^{\mathrm{LIF}}\right\rangle$ denotes population averaged spike rates of the MC and LIF neuron network populations, respectively. $S_{\left\langle\nu_{X}\right\rangle\left\langle\nu_{X}\right\rangle}(f)$ and $S_{\left\langle\nu_{X}^{\mathrm{LF}}\right\rangle\left\langle\nu_{X}^{\mathrm{LF}}\right\rangle}(f)$ denotes population firing rate autospectral power at each frequency $f$ (see Section 2.5 for details). For this minimization problem we used an initial population size of 100, and ran the algorithm for 20 generations with default parameters.

The pseudo-weight vector approach (Deb 2001; Blank and Deb 2020) is used to select a solution from the solution set that perform well with respect to all objective functions. The pseudo weight, a normalized distance measure from the 
Table 2: Synaptic parameters for recurrent network (continued from Table 1). Capped and discrete distributions are normalized such that the integral or sum over all values equals 1 .

\begin{tabular}{|c|c|c|}
\hline Symbol & Value/definition & Description \\
\hline$\widetilde{G}_{\text {syn } Y X}$ & $\left\{\begin{array}{l}\mathcal{N}(0.15 \mathrm{nS}, 0.02 \mathrm{nS}) \Theta(G) \text { for } X=\mathrm{E}, Y=\mathrm{E} \\
\mathcal{N}(0.125 \mathrm{nS}, 0.0125 \mathrm{nS}) \Theta(G) \text { for } X=\mathrm{E}, Y=\mathrm{I} \\
\mathcal{N}(4.5 \mathrm{nS}, 0.45 \mathrm{nS}) \Theta(G) \text { for } X=\mathrm{I}, Y=\mathrm{E}\end{array}\right.$ & Synaptic conductance distributions \\
\hline$E_{X}$ & $\begin{array}{c}\mathcal{N}(2.0 \mathrm{nS}, 0.2 \mathrm{nS}) \Theta(G) \text { for } X=\mathrm{I}, Y=\mathrm{I} \\
0 \mathrm{mV} \text { for } X=\mathrm{E},-80 \mathrm{mV} \text { for } X=\mathrm{I} \\
\left(e^{-\left(t-t_{\mathrm{s}}\right) / \tau_{1}}-e^{-\left(t-t_{\mathrm{s}}\right) / \tau_{2}}\right)\end{array}$ & Synaptic reversal potential \\
\hline$f_{Y X}(t)$ & $\begin{array}{c}\left(\frac{e}{e^{-\tau_{\text {peak }} / \tau_{1}}-e^{-\tau_{\text {peak }} / \tau_{2}}}\right) \Theta\left(t-t_{\mathrm{s}}\right) \\
\text { where } \tau_{\text {peak }}=\frac{\tau_{2} \tau_{1}}{\tau_{2}-\tau_{1}} \log \left(\frac{\tau_{2}}{\tau_{1}}\right)\end{array}$ & Synaptic temporal kernel \\
\hline$\tau_{1}$ & $0.2 \mathrm{~ms}$ for $X=\mathrm{E}, 0.1 \mathrm{~ms}$ for $X=\mathrm{I}$ & Synaptic rise time constant \\
\hline$\tau_{2}$ & $\begin{array}{c}1.8 \mathrm{~ms} \text { for } X=\mathrm{E}, 9.0 \mathrm{~ms} \text { for } X=\mathrm{I} \\
(\mathcal{N}(1.5 \mathrm{~ms}, 0.3 \mathrm{~ms}) \Theta(t-0.3 \mathrm{~ms}) \text { for } X=\mathrm{E}, Y=\mathrm{E}\end{array}$ & Synaptic decay time constant \\
\hline$\widetilde{\Delta}_{Y X}$ & $\left\{\begin{array}{l}\mathcal{N}(1.4 \mathrm{~ms}, 0.4 \mathrm{~ms}) \Theta(t-0.3 \mathrm{~ms}) \text { for } X=\mathrm{E}, Y=\mathrm{I} \\
\mathcal{N}(1.3 \mathrm{~ms}, 0.5 \mathrm{~ms}) \Theta(t-0.3 \mathrm{~ms}) \text { for } X=\mathrm{I}, Y=\mathrm{E}\end{array}\right.$ & Conduction delay distributions \\
\hline$L_{Y X}(z)$ & $\begin{array}{l}(\mathcal{N}(1.2 \mathrm{~ms}, 0.6 \mathrm{~ms}) \Theta(t-0.3 \mathrm{~ms}) \text { for } X=\mathrm{I}, Y=\mathrm{I} \\
\qquad \begin{array}{l}\frac{\mathcal{N}(0,100)(z)}{3}+\frac{2 \mathcal{N}(500,100)(z)}{3} \\
\quad X=\mathrm{E}, Y=\mathrm{E}, \mathcal{S} \backslash\{\mathrm{soma}\} \\
\mathcal{N}(50,100)(z), X=\mathrm{E}, Y=\mathrm{I}, \mathcal{S} \backslash\{\mathrm{soma}\} \\
\mathcal{N}(-50,100)(z), X=\mathrm{I}, Y=\mathrm{E} \\
\mathcal{N}(-100,100)(z), X=\mathrm{I}, Y=\mathrm{I}\end{array}\end{array}$ & Depth-dependence, syn. density \\
\hline $\bar{G}_{\text {syn } Y \text { ext }}$ & $0.2 \mathrm{nS}$ & External synapse conductance \\
\hline$E_{\text {ext }}$ & $0 \mathrm{mV}$ & Ext. synapse rev. potential \\
\hline$f_{Y \operatorname{ext}}(t)$ & $f_{Y X}(t)$ & Ext. synapse temporal kernel \\
\hline$\tau_{1}$ & $0.2 \mathrm{~ms}$ & Ext. synapse rise time constant \\
\hline$\tau_{2}$ & $1.8 \mathrm{~ms}$ & Ext. synapse decay time constant \\
\hline$k_{Y \text { ext }}$ & $\{465,160\}$ & \# ext. synapses per neuron \\
\hline$\left\langle\nu_{\text {ext }}\right\rangle$ & $40 \mathrm{~s}^{-1}$ (Poisson statistics) & Ext. syn. activation rate \\
\hline$\widetilde{\Delta}_{Y \text { ext }}$ & $\delta(t)$ & Ext. syn. conduction delay \\
\hline$L_{Y \text { ext }}$ & 1 & Ext. syn. depth dependence \\
\hline
\end{tabular}

Table 3: Measurement and simulation parameters for recurrent network (continued from Table 2).

\begin{tabular}{|c|c|c|}
\hline Symbol & Value/definition & Description \\
\hline$\Delta t$ & $0.0625 \mathrm{~ms}$ & Temporal step size \\
$t_{\text {sim }}$ & $12000 \mathrm{~ms}$ & Simulation duration \\
$t_{\text {transient }}$ & $2000 \mathrm{~ms}$ & Startup transient duration \\
$C^{\circ}$ & $34^{\circ} \mathrm{C}$ & Simulation temperature \\
$V_{\mathrm{m}}(0)$ & $-65 \mathrm{mV}$ & Initial membrane voltage \\
\hline$s_{u}(t)$ & $\sum_{k} \delta_{t_{k}}$ for all $u \in X$ & Spike times $t_{k}$ of neurons $u$ \\
\hline $\mathbf{x}_{\text {ch. }}=\mathbf{y}_{\text {ch. }}$ & {$[0, \ldots, 0]$} & $x, y$-positions of electrode contacts \\
$\mathbf{z}_{\mathrm{ch} .}$ & {$[1000,900, \ldots,-100,-200] \mu \mathrm{m}$} & $z$-positions of electrode contacts \\
$\mathbf{R}$ & {$\left[\mathbf{x}_{\mathrm{ch} .}, \mathbf{y}_{\mathrm{ch} .}, \mathbf{z}_{\mathrm{ch} .}\right]^{\top}$} & 5 Electrode contact locations \\
$r_{\text {contact }}$ & $5 \mu \mathrm{m}$ & Electrode contact radiis \\
$\mathbf{n}_{\text {contact }}$ & {$[0,1,0]$} & Electrode contact surface normals \\
$\sigma$ & $0.3 \mathrm{~S} \mathrm{~m}{ }^{-1}$ & Conductivity \\
$\psi$ & $\left\{V_{\mathrm{e}}(\mathbf{R}, t), \mathbf{P}(t)\right\}$ & Predicted signals \\
\hline
\end{tabular}


worst solution for each objective function $F_{i}$, is herein calculated as:

$$
w_{i}=\frac{\left(F_{i}^{\max }-F_{i}(\mathbf{x})\right) /\left(F_{i}^{\max }-F_{i}^{\min }\right)}{\sum_{m=1}^{M}\left(F_{m}^{\max }-F_{m}(\mathbf{x})\right) /\left(F_{m}^{\max }-F_{m}^{\min }\right)} .
$$

$F_{i}^{\max }$ and $F_{i}^{\min }$ denotes the maximum and minimum value of $F_{i}(\mathbf{x})$ in the last generation, respectively. Then, the best-fit parameter vector $\mathbf{x}$ where chosen as the one that minimized $\left\|\left[w_{1}, w_{2}, w_{3}, w_{4}\right]^{\top}-[0.25,0.25,0.25,0.25]^{\top}\right\|$. We here use the implementation provided by the pymoo.factory.get_decision_making method.

The network is implemented and simulated in NEST (Gewaltig and Diesmann 2007; Deepu et al. 2021) using exact integration for step size $\Delta t$ (Rotter and Diesmann 1999).

Table 4: LIF network and neuron parameters. Capped and discrete distributions are normalized such that the integral or sum over all values equals 1 .

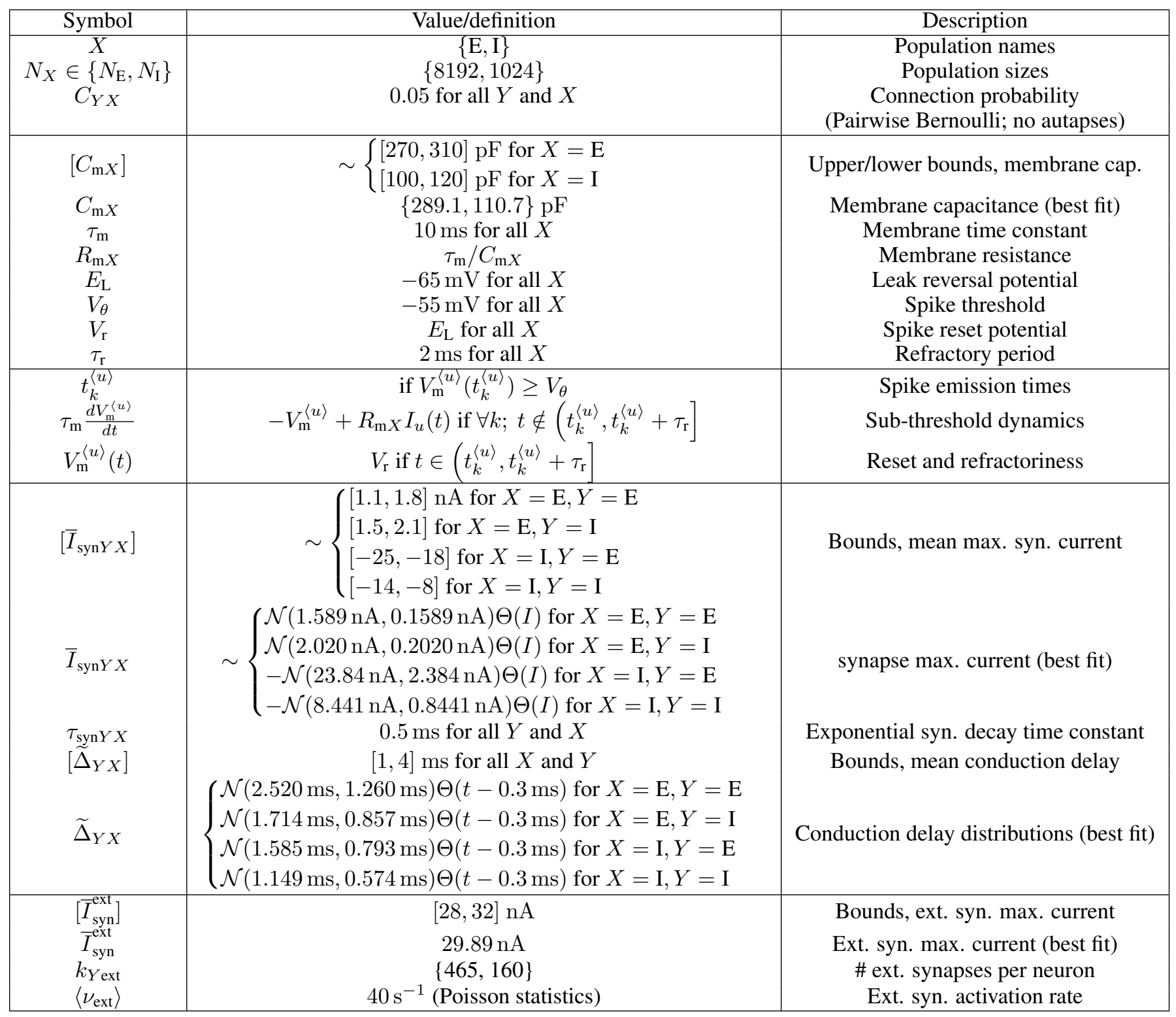




\subsection{Hybrid scheme for extracellular signals}

\subsubsection{Two-step method}

The so-called 'hybrid scheme' (Hagen et al. 2016) is a proposed solution for computing extracellular signals from spiking activity in recurrent neuron network models. This scheme is hybrid in the sense that spiking activity is first simulated separately and stored, then recorded spike events are loaded and used for synaptic activation times in corresponding populations of MC neuron models set up to predict extracellular signals. The latter step negates the need for recurrent connections and spike communication between $\mathrm{MC}$ neurons, thus the problem of computing signals can be solved in an embarrassingly parallel manner.

Here, we incorporate this core idea of the hybrid scheme by storing population geometries, spikes and the full synaptic connectome (placements, weights, conduction delays, pre- and postsynaptic neuron IDs) of the recurrent MC neuron networks to file, and reinstate synaptic placements and synaptic activation times in separate simulations but without actual recurrent connections. This step allows for computing signals identical to the recurrent model in case the MC neuron models are those of the recurrent network, but here, we shall rely on models where the membrane and synapse dynamics are approximated by linear dynamics. Thus in this version of the hybrid scheme only signal contributions that stem from synapse activations on postsynaptic neurons is accounted for in predicted signals, while contributions by presynaptic APs are not. The scheme thus lends itself to predictions of signals thought to mainly stem from synaptic activity, that is, LFP, ECoG, EEG and MEG signals.

\subsubsection{Linear approximations}

Here, we describe the different linear approximations to the different constituents of the original non-linear model that contribute to the extracellular signals as implemented via the following steps:

1. Approximate conductance-based synapses by equivalent current-based synapses:

$$
\begin{aligned}
I_{\mathrm{syn} Y X}(t) & =\bar{G}_{\mathrm{syn} Y X} f_{Y X}(t)\left(V_{\mathrm{m}}(t)-E_{X}\right) \\
& \approx \bar{G}_{\mathrm{syn} Y X} f_{Y X}(t)\left(\left\langle V_{\mathrm{m}}(t)\right\rangle-E_{X}\right) \\
& =\bar{I}_{\operatorname{syn} Y X} f_{Y X}(t),
\end{aligned}
$$

where $\left\langle V_{\mathrm{m}}(t)\right\rangle$ denotes the typical postsynaptic potential (or its expectation value). Here, we recorded somatic potentials in a subset of neurons in each population of the recurrent MC neuron networks and let $\left\langle V_{\mathrm{m}}(t)\right\rangle$ equate the median somatic potentials.

2. As a separate step, active ion channel currents on the specific form

$$
i_{\mathrm{w}}(t)=-\bar{g}_{\mathrm{w}} \omega(t)\left(V_{\mathrm{m}}(t)-E_{\mathrm{w}}\right)
$$

is approximated by equivalent, linearly dependent currents similar to Remme and Rinzel 2011; Ness et al. 2016. Here, $E_{\mathrm{w}}$ is the channel reversal potential and $\omega(t)$ the gating variable which dynamics are given in terms of an activation time function $\tau_{\mathrm{w}}(V)$ and activation function $\omega_{\infty}(V)$ as

$$
\tau_{\mathrm{w}}\left(V_{\mathrm{m}}(t)\right) \frac{\partial \omega(t)}{\partial t}=\omega_{\infty}\left(V_{\mathrm{m}}(t)\right)-\omega(t)
$$

If the voltage dynamics of the active compartment is defined by

$$
c_{\mathrm{m}} \frac{\partial V_{\mathrm{m}}(t)}{\partial t}=-\bar{g}_{\mathrm{L}}\left(V_{\mathrm{m}}(t)-E_{\mathrm{L}}\right)-\bar{g}_{\mathrm{w}} \omega(t)\left(V_{\mathrm{m}}(t)-E_{\mathrm{w}}\right)+I_{\mathrm{a}},
$$

one can obtain the so-called quasi-active approximation (Koch 1984; Remme and Rinzel 2011; Ness et al. 2016) by linearizing each voltage dependent term around the steady state value $\left\langle V_{\mathrm{m}}(t)\right\rangle$ resulting in

$$
\begin{aligned}
c_{\mathrm{m}} \frac{\partial V_{\mathrm{m}}(t)}{\partial t} & =-\bar{g}_{\mathrm{L}}\left(\gamma_{\mathrm{R}}\left(V_{\mathrm{m}}(t)-\left\langle V_{\mathrm{m}}(t)\right\rangle\right)+\eta m(t)\right)+I_{\mathrm{a}}, \text { where } \\
\gamma_{\mathrm{R}} & =1+\frac{\bar{g}_{\mathrm{w}} \omega_{\infty}\left(\left\langle V_{\mathrm{m}}(t)\right\rangle\right)}{\bar{g}_{\mathrm{L}}}, \text { and } \\
\eta & =\frac{\bar{g}_{\mathrm{w}}\left(\left\langle V_{\mathrm{m}}(t)\right\rangle-E_{\mathrm{w}}\right)}{\bar{g}_{\mathrm{L}}} \frac{\partial \omega_{\infty}\left(\left\langle V_{\mathrm{m}}(t)\right\rangle\right)}{\partial V_{\mathrm{m}}} .
\end{aligned}
$$

Here, an equivalent gating variable is defined as

$$
m(t)=\left(\omega(t)-\omega_{\infty}\left(\left\langle V_{\mathrm{m}}(t)\right\rangle\right)\right) / \frac{\partial \omega_{\infty}\left(\left\langle V_{\mathrm{m}}(t)\right\rangle\right)}{\partial V_{\mathrm{m}}},
$$


and its linear dynamics is governed by

$$
\tau_{\mathrm{w}}\left(\left\langle V_{\mathrm{m}}(t)\right\rangle\right) \frac{\partial m(t)}{\partial t}=V_{\mathrm{m}}(t)-\left\langle V_{\mathrm{m}}(t)\right\rangle-m(t) .
$$

Above, $\gamma_{\mathrm{R}}$ denotes the ratio between the total and leak conductance, while $\eta$ characterize whether the quasiactive current approximation acts as positive $(\eta<0)$ or negative $(\eta>0)$ feedback. For the special case $\eta=0$ the quasi-active current is 'frozen', acting as a passive current (Ness et al. 2016). Note that the above sets of equations corresponds to channels usually modeled with a single state variable (e.g., $\mathrm{I}_{\mathrm{h}}$-type currents), but generalize also to current types with more than one gating variable (e.g., $\mathrm{Na}^{+}$- and $\mathrm{K}^{+}$type currents), see (Remme and Rinzel 2011) for details.

3. With linearized active ion channels, the leak conductance $E_{\mathrm{L}}$ is further modified as

$$
E_{\mathrm{L}}^{\mathrm{mod}}=\left\langle V_{\mathrm{m}}(t)\right\rangle+\sum_{\mathrm{w}} \frac{\bar{g}_{\mathrm{w}}\left(\left\langle V_{\mathrm{m}}(t)\right\rangle-E_{\mathrm{w}}\right)}{\bar{g}_{\mathrm{L}}},
$$

which ensures that the resting potential of the quasi-active model is similar to $\left\langle V_{\mathrm{m}}(t)\right\rangle$. We note that this modification do not affect extracellular signal predictions where current-based synapses (pt. 1) are used, but is applied anyway as in Ness et al. 2016.

4. In principle one may remove active ion channels omitting the above linearization tricks altogether if their net contributions to the total transmembrane currents can be assumed to be minuscular around typical membrane voltage values. Here however we do account for all channels.

5. With current-based synapses, optionally incorporate the effective membrane conductance $g_{\mathrm{eff}}$ which amounts to a modified passive leak conductivity. Assuming the total membrane conductance depends only on synaptic currents of recurrent and external connections and the passive leakage current, the total membrane conductivity per postsynaptic compartment $m$ is of postsynaptic neuron indexed by $v$ is

$$
g_{m}(t)=\bar{g}_{\mathrm{L}}+\frac{1}{A_{m}} \sum_{X^{\prime} \in X \cup\{\operatorname{ext}\}} \sum_{u \subset X^{\prime}} \bar{G}_{\mathrm{syn} v u}\left(f_{Y X^{\prime}} * s_{u} * \delta_{\Delta_{v u}}\right)(t)
$$

where $\bar{g}_{\mathrm{L}}$ is the specific passive leak conductance, $A_{m}$ compartment area, $s_{u}(t)$ the sequence of presynaptic spikes and $\delta_{\Delta_{v u}}=\delta\left(t-\Delta_{v u}\right)$ the conduction delay. The asterisk symbol $(*)$ denotes a temporal convolution. The double sum over presynaptic populations $X^{\prime} \in X \cup\{$ ext $\}$ and units $u \in X^{\prime}$ implies that each presynaptic unit $u$ targeting the compartment is accounted for. We introduce this notation to express that also synapses from external sources ('ext') must be accounted for. Assuming a fixed average presynaptic spike rate $\left\langle\nu_{u}\right\rangle=$ $\Delta t^{-1} \int_{t}^{t+\Delta t} s_{u}(t) d t$ for any time duration $\Delta t$ and a normalized delay distribution (where $\int_{0}^{\infty} \widetilde{\Delta}_{Y X}(t) d t=1$ ), the time-averaged effective conductance in each compartment $m$ is approximately

$$
g_{\mathrm{eff} m}=\left\langle g_{m}(t)\right\rangle \approx \bar{g}_{\mathrm{L}}+\frac{1}{A_{m}} \sum_{X^{\prime} \in X \cup\{\mathrm{ext}\}} \sum_{u \subset X^{\prime}}\left\langle\nu_{u}\right\rangle \bar{G}_{\mathrm{syn} v u} \int_{0}^{\infty} f_{Y_{X^{\prime}}}(t) d t .
$$

Then, the original $\bar{g}_{\mathrm{L}}$ value may be replaced by $g_{\mathrm{eff} m}$ on a per-compartment basis.

\subsection{Kernel based methods}

\subsubsection{Hybrid model kernels}

The cable equation describing the membrane voltage dynamics across different neuron sections is by itself a linear partial differential equation. However, voltage-dependent dynamics of synapses and ion channels combined with the cable equation constitutes a non-linear system. Applying pts. 1-5 in Section 2.2.2 to this system however allows for computing a linear relation between spikes of each presynaptic neurons and resulting contribution to the extracellular signal(s), as every component including synaptic conductances and voltage-gated ion channels of this hybridized simulation scheme is then linearized and separable with respect to time and space. The different effects of transmission delay distributions, evoked synaptic currents, filtering of inputs by dendrites plus quasi-active approximation of active ion channels and linear forward model may then be combined into a spatiotemporal kernel $H_{v u}(\mathbf{R}, \tau)$ where $\tau$ denote time lag relative to presynaptic spike times in $s_{u}(t)$ (Hagen et al. 2016). $u$ and $v$ denote pre- and postsynaptic neuron indices, respectively. One may consider computing this filter as

$$
\begin{aligned}
H_{v u}(\mathbf{R}, \tau) & =\mathcal{F}_{v}\left[\bar{I}_{\operatorname{syn} Y X}\left(\delta_{\Delta_{v u}} * f_{Y X} * \mathbf{G}_{v u}\right)(\mathbf{r}, \tau)\right] \\
& =\mathcal{F}_{v}\left[I_{\mathrm{m}}\left(\mathbf{r}_{m}, \tau\right)\right]
\end{aligned}
$$


where the Green's like functions $\mathbf{G}_{v u}(\mathbf{r}, \tau)$ encompass all dendritic filtering effects on delta-pulse synaptic input currents that when convolved by the synaptic kernel and delay distribution and scaled by the synaptic current amplitude yield a distribution of compartmental transmembrane currents $\left[I_{\mathrm{m}}\left(\mathbf{r}_{m}, \tau\right)\right] . \mathcal{F}_{v}=\mathcal{F}_{v}(\mathbf{R}, \mathbf{r})$ denotes a linear mapping of transmembrane currents of postsynaptic neuron $v$ to corresponding signal measurement locations $\mathbf{R}$. If each kernel function which is specific to each pair of pre- and postsynaptic neuron can be computed, one could compute the signal as the sum over convolutions between presynaptic spike times and corresponding kernels as

$$
\psi(\mathbf{R}, t)=\sum_{X} \sum_{Y} \sum_{u \in X} \sum_{v \subset Y}\left(s_{u} * H_{v u}\right)(\mathbf{R}, t) .
$$

In large networks however, computing the full set $H_{v u}(\mathbf{R}, \tau)$ can become intractable as the number of connected pairs of neurons (and consequently the number of kernels) grow proportionally to the product $N_{X} N_{Y}$ if the connection probabilities are kept fixed. However, Hagen et al. 2016 show that a good approximation to the signal $\psi(\mathbf{R}, t)$ can be obtained by measuring kernels averaged over all pre- and postsynaptic neurons in each population $X$ and $Y$. In order to compute these kernel averages directly using the 'hybrid scheme', actual network spiking activity is first replaced by simultaneous and deterministic events $s_{u}(t)=\delta_{t_{X}}$ where $t_{X}>0$ is a chosen time for each population $X$, compute via the disassociated network model the signal contributions of each postsynaptic population $\psi_{Y X}(\mathbf{R}, t)$ around $t_{X}$ and average the response as

$$
H_{Y X}(\mathbf{R}, \tau)=\frac{1}{N_{X}} \psi_{Y X}(\mathbf{R}, \tau)
$$

where $\tau$ denote time relative to $t_{X}$. Thus these kernels must be causal, that is, by construction $H_{Y X}(\mathbf{R}, \tau)=0$ for $\tau<0$ as any contribution to the signal $\psi(\mathbf{R}, t)$ is solely postsynaptic which in this scenario can not occur before the presynaptic spike event at $\tau=0$ plus the conduction delay ${ }^{3}$. We let the computed kernels span the interval $\tau \in\left[-\tau_{\max }, \tau_{\max }\right]$, where $\tau_{\max }$ denotes a maximum lag value. The postsynaptic responses typically decay to approximately zero after a few tens of milliseconds. This decay time is related to the time constants relevant to the neuronal dynamics (that is, $\tau_{\mathrm{m}}, \tau_{\mathrm{syn}}, \tau_{\mathrm{w}}, \ldots$ ). Throughout this manuscript, we chose $\tau_{\max }=100 \mathrm{~ms}$ for computed kernels, which we assume is a few multiples of relevant time constants.

Finally, if we define the presynaptic population spiking activity as the sequence of Dirac delta functions $s_{X}(t)=$ $\sum_{u \in X} s_{u}(t)$, the final signal approximation $\hat{\psi}(\mathbf{R}, t) \approx \psi(\mathbf{R}, t)$ may then be computed as the sum over convolutions

$$
\hat{\psi}(\mathbf{R}, t)=\sum_{X} \sum_{Y}\left(s_{X} * H_{Y X}\right)(\mathbf{R}, t) .
$$

As we more often deal with spike events and sampled signals on a discrete time grid, it is convenient to redefine the spike sequences $s_{X}(t)$ as spike rates by the temporal binning

$$
\nu_{X}\left[t_{i}\right]=\Delta t^{-1} \int_{t_{i}-\Delta t / 2}^{t_{i}+\Delta t / 2} s_{X}(t) d t
$$

where $\Delta t$ denotes the simulation step size. Then, the above equation can be written as

$$
\hat{\psi}(\mathbf{R}, t)=\sum_{X} \sum_{Y}\left(\nu_{X} * H_{Y X}\right)(\mathbf{R}, t) .
$$

Note that a valid implementation alternative to the linear convolutions detailed here is applying the kernels using a linear filter function implementation. The measured kernels are essentially finite impulse responses (FIR) as they are 0 for all time lags $\tau<\Delta t$. Also note that all signals considered here are simulated and on a discrete time grid with resolution $\Delta t$, thus corresponding filter and convolution operations are discrete (see Section 2.5).

\subsubsection{Kernel predictor}

Different from the 'hybrid scheme' kernel method described above, the main aim of this work is to develop a method to directly compute a set of deterministic kernels $\hat{H}_{Y X}(\mathbf{R}, \tau)$ needed for all connection pathways between pre- and postsynaptic populations $X$ and $Y$, based on some expectation values for cell and synaptic placements and other network parameters. Our aim is to replace simulations of populations of $\mathrm{MC}$ neurons via the hybrid scheme with a single MC neuron simulation per kernel. Thus the number of MC neuron simulations corresponds to the number of pathways between any population $X$ and $Y$ which is significantly less than the total neuron count in each network. First, let us in general terms assume that:

\footnotetext{
${ }^{3}$ Note that we have chosen to ignore contributions by presynaptic activity, that is, transmembrane currents of presynaptic neurons from APs and axonal propagation.
} 
1. The dynamics of the neuronal cables and synaptic input can be approximated as a linear system (resulting from the same steps as in Section 2.2.2).

2. Each postsynaptic population can be represented by one typical biophysically detailed neuron model. Effectively, the whole postsynaptic neuron population is collapsed to a single neuron with linearized membranes receiving all inputs, while the effect of the spatial distribution of cells in space is accounted for via the VC forward model (see Section 2.4.2).

3. The underlying statistics of synaptic placements and currents is preserved, which allows us to compute the average synaptic current density for each recurrent connection over the whole postsynaptic population 'neuron'.

4. Accounting for the distribution of neurons along the $z$-axis and ignoring their radial location, we let the synaptic density be proportional to the membrane area of postsynaptic compartments $A_{m}$ multiplied by a function $\mathcal{L}_{Y X}(z)$ obtained as the convolution of $L_{Y X}(z)$ (see Table 2) and the $z$-component of $\widetilde{\mathbf{r}}_{Y}$. Hence we compute the expectation value for synaptic in-degree per compartment indexed by $m$ as

$$
\left\langle k_{\mathrm{syn} Y X m}\right\rangle=\frac{\left\langle\widetilde{k}_{Y X}\right\rangle K_{Y X}}{N_{Y}} \frac{\mathcal{L}_{Y X}\left(z_{m}\right) A_{m}}{\sum_{m} \mathcal{L}_{Y X}\left(z_{m}\right) A_{m}},
$$

where $z_{m}$ denotes the midpoint location of each compartment projected on the $z$-axis, and $\left\langle\widetilde{k}_{Y X}\right\rangle$ the mean multapse count per connection. With this quantity one may define the per-compartment synaptic input per activation as

$$
I_{\mathrm{syn} Y X m}(t)=N_{X}\left\langle k_{\mathrm{syn} Y X m}\right\rangle \bar{I}_{\mathrm{syn} Y X} f_{Y X}(t) .
$$

As above, the term $f_{Y X}(t)$ denotes the temporal component of synapse currents for each connection.

5. Optionally accounting for the effective leak conductivity, Equation (18) must be modified per compartment as

$$
g_{\mathrm{eff} m}=\bar{g}_{\mathrm{L}}+\frac{1}{A_{m}} \sum_{X^{\prime} \in X \cup\{\mathrm{ext}\}}\left\langle\nu_{X^{\prime}}\right\rangle\left\langle k_{\mathrm{syn} Y X^{\prime} m}\right\rangle \bar{G}_{\mathrm{syn} Y X^{\prime}} \int_{0}^{\infty} f_{Y X^{\prime}}(t) d t .
$$

As above, we account for the external population 'ext' jointly with the main network populations in $X$.

6. Then one may straightforwardly compute the resulting postsynaptic response, that is, the full set of transmembrane currents $\left[I_{\mathrm{m}}\left(\mathbf{r}_{m}, \tau\right)\right]$, by applying synaptic currents $I_{\mathrm{syn} Y X m}(\tau)$ in a single MC neuron simulation for all connections between populations $X$ and $Y$. In order to temporarily compute the approximated kernel functions $\hat{H}_{Y X}^{\text {temp }}(\mathbf{R}, \tau)$ for different extracellular signals, the resulting transmembrane currents must be combined with appropriate forward model matrices $\mathcal{F}$ calculated as described below.

7. Finally to account for network conduction delay distributions, the intermediate kernels must be filtered in the temporal domain as

$$
\hat{H}_{Y X}(\mathbf{R}, \tau)=\left(\widetilde{\Delta}_{Y X} * \hat{H}_{Y X}^{\mathrm{temp}}\right)(\mathbf{R}, \tau) .
$$

\subsection{Forward models}

\subsubsection{Forward models for MC neurons and populations}

As derived from volume conductor theory, the different electric and magnetic signals that can be computed from the electric activity of brain cells are linearly dependent on transmembrane currents (or axial currents) (see e.g., Einevoll et al. 2013 and references therein). Thus, some arbitrary signals $\psi\left(\mathbf{R}_{n}, t\right)$ in $M$ different spatial locations $\mathbf{R}_{n}$ (or directions in case of current dipole moments) from $N$ compartmental sources indexed by $m$ located at $\mathbf{r}_{m}$ can be computed as

$$
\left[\psi\left(\mathbf{R}_{n}, t\right)\right]=\mathcal{F}\left[I_{\mathrm{m}}\left(\mathbf{r}_{m}, t\right)\right]
$$

where $\mathcal{F}$ is a matrix with dimensions $(M, N)$ wherein each element $f_{n m}$ is the chosen forward solution mapping the contribution from each source to the corresponding measurement. $\left[I_{\mathrm{m}}\left(\mathbf{r}_{m}, t\right)\right]$ denotes the transmembrane currents of compartments $m$. A simple linear forward model mapping transmembrane currents of an $N$-compartment model to extracellular potentials in $M$ locations would be the point-source formalism, which models neuronal sources and measurement sites as infinitesimally small points in an infinite homogeneous, isotropic and linear volume conductor with conductivity $\sigma$. The point-source formula (Lindén et al. 2014, Eq. (2)) results in elements

$$
f_{n m}=\frac{1}{4 \pi \sigma} \frac{1}{\left\|\mathbf{R}_{n}-\mathbf{r}_{m}\right\|} .
$$


For the presently used line sources (Lindén et al. 2014, Eq. (4)), the elements of $\mathcal{F}$ are calculated as

$$
f_{n m}=\frac{1}{4 \pi \sigma \Delta s_{n m}} \log \left|\frac{\sqrt{h_{n m}^{2}+\rho_{n m}^{2}}-h_{n m}}{\sqrt{\ell_{i}^{2}+\rho_{n m}^{2}}-\ell_{n m}}\right|,
$$

where $\rho_{n m}$ is the distance perpendicular to line source (compartment) $m, h_{n m}$ the longitudinal distance from the end of the line source and $\ell_{n m}=\Delta s_{n m}+h_{n m}$ the longitudinal distance from the start of the line source with length $\Delta s_{n m}$ to some electrode contact located at $\mathbf{R}_{n}$.

To mimic the finite extent of contacts of experimental recording electrodes, we apply the 'disk-electrode' approximation to the extracellular potential (Lindén et al. 2014, Eq. 6) by embedding averaged values of $f_{n m}$ from Equation (32) for 100 random locations within radius $r_{\text {contact }}$ into $\mathcal{F}$.

The approach is applicable also to other types of measurements which are linearly dependent on the transmembrane current sources, such as the current dipole moment (Lindén et al. 2010). For computing the current dipole moment $\mathbf{P}$ the columns of $\mathcal{F}$ are simply

$$
f_{m}=\mathbf{r}_{m}=\left[\begin{array}{l}
x_{m} \\
y_{m} \\
z_{m}
\end{array}\right]
$$

where $\left(x_{m}, y_{m}, z_{m}\right)$ denotes the midpoint coordinates of each compartment.

\subsubsection{Modified forward models for deterministic kernel predictions}

When computing extracellular signals via the kernel approximation scheme we must account for the distributions of cells in space. Here we assume that each population is radially symmetric around the vertical $z$-axis, homogeneous within some radius $R$ and inhomogeneous along the $z$-axis as described by a probability density function $\widetilde{\mathbf{r}}_{X}$ (see Table 1). In order to compute extracellular potentials, we use the analytical forward solution for the electric potential from a planar disk with homogeneous current density (Pettersen et al. 2006)

$$
a_{n m}\left(z_{n}, z_{m}\right)=\frac{1}{2 \sigma \pi R^{2}}\left(\sqrt{\left(z_{n}-z_{m}\right)^{2}+R^{2}}-\left|z_{n}-z_{m}\right|\right),
$$

which is subsequently convolved with the depth-dependence of cell placement $g(z)=\widetilde{\mathbf{r}}_{X} \cdot \mathbf{e}_{z}$ (where $\mathbf{e}_{z}$ denotes the unit vector along the $z$-axis), resulting in matrix elements:

$$
f_{n m}=\int_{-\infty}^{\infty} a_{n m}\left(z_{n}-z, z_{m}\right) g(z) d z
$$

Here, we solve this convolution integral numerically using the quad method of the scipy. integrate module. Note that we apply the same equation also when predicting kernels for the biophysically detailed neuron network (see Section 2.1.3).

For computing the current dipole moment with radial symmetry around the $z$-axis the mapping matrix' columns are simply modified as

$$
f_{m}=\mathbf{r}_{m}=\left[\begin{array}{c}
0 \\
0 \\
z_{m}
\end{array}\right]
$$

where $z_{m}$ denotes the midpoint coordinates of each compartment along the $z$-axis. We do not account for the distribution of cells along the $z$-axis as it does not affect the current dipole moment. Due to radial symmetry, the components in the lateral directions are expected to cancel (Hagen et al. 2018; Næss et al. 2021), hence the corresponding matrix elements are set to zero.

\subsection{Signals and signal analysis methods}

Throughout this study, the different signals we consider are: somatic membrane potentials $V_{\mathrm{m}}^{\text {soma }}(t)$; spike trains $s_{u}(t)$; population firing rates $\nu_{X}(t)$ obtained by counting spikes per time bin of width $\Delta t$ divided by bin width providing a signal with unit spikes $\mathrm{s}^{-1}$ as defined in Equation (24); and raw and low-pass filtered extracellular signals $\psi\left(\mathbf{R}_{n}, t\right)$ (extracellular potentials $V_{\mathrm{e}}\left(\mathbf{R}_{n}, t\right)$; current dipole moments $\mathbf{P}(t)$ ). For extracellular signals we consider only frequencies $f>0 \mathrm{~Hz}$ by subtracting the mean value in each channel for times $t>t_{\text {transient }}$. For low-pass filter operations we used elliptic (Cauer) digital filter design. Here, we used filters of the 2 nd order with $0.1 \mathrm{~dB}$ maximum ripple in the pass band, minimum attenuation of $40 \mathrm{~dB}$ in the stop band and a critical (cutoff) frequency of $100 \mathrm{~Hz}$. Filter coefficients were generated using the scipy.signal.ellip function with parameter output='sos' (second-order sections). 
The low-pass filter was applied to the data using the scipy.signal.sosfiltfilt function which implements a forward-backward (zero time-lag) filter operation.

In order to quantify relative differences in amplitudes of approximated signals $x(t)$ and ground truth $y(t)$ we defined the 'ratio of standard deviations' as

$$
r_{\mathrm{STD}}=\frac{\operatorname{STD}(x)}{\operatorname{STD}(y)} .
$$

In order to quantify temporal agreement with signals $x(t)$ and $y(t)$ we computed the squared correlation coefficient (coefficient of determination) $R^{2}$ at zero time lag as:

$$
R^{2}=\frac{\operatorname{COV}(x, y)}{\operatorname{VAR}(x) \operatorname{VAR}(y)} .
$$

In order to aggregate our $R^{2}$ and $r_{\mathrm{STD}}$ metrics for signals computed at different depths, we computed the 10th and 90th percentiles using the implementation of numpy . quantile with quantiles equal to 0.1 and 0.9 , respectively.

For convolutions, we use the discrete convolution between vectors $x$ and $y$ defined as:

$$
(x * y)[k]=\sum_{l=-\infty}^{\infty} x[l] y[k-l] .
$$

Here, we used the implementation provided by numpy. convolve with mode=' same'.

Application of discrete FIR filter coefficients $h$ to a signal $x$ (relevant for NEST predictions) is defined as

$$
y[k]=\sum_{l=0}^{L} h[l] x[k-L] .
$$

Estimates of power spectra $P_{x x}(f)$ of signals $x(t)$ use the Welch's average periodogram method (Welch 1967) as implemented by scipy. signal. welch. Unless specified otherwise, we use the periodogram settings nfft $=2048$, noverlap $=1536$, $\mathrm{f} s=\Delta t^{-1}$ (in $\mathrm{Hz}$ ) and detrend=False. When optimizing point-neuron network parameters we used the setting detrend=' constant'.

\subsection{NESTML FIR filter extension}

In order to incorporate extracellular signal predictions using the computed sets of causal kernels from a point-neuron simulation in NEST (see Section 2.1.4), a finite impulse response (FIR) filter implementation of Equation (40) is now expressed in the NESTML modeling language (Plotnikov et al. 2016; Nagendra Babu et al. 2021). The FIR filter model is written as a neuron model in NESTML, which takes neuronal spikes as input and computes the filter output while the simulation progresses. The output can then be queried and recorded to file using standard NEST devices. The NESTML toolchain generates $\mathrm{C}++$ code for the model, which is compiled into a NEST extension module, allowing the FIR filter node (or a heterogeneous population of filter nodes) to be instantiated in NEST simulations.

As per Equation (40), the FIR filter model defines $L$ as the order of the filter and $h$ as a vector of length $L$ containing the filter coefficients. The values of $L$ and $h$ can be set externally from the simulation script, and in this study we insert filter coefficients from each set of predicted kernels $\hat{H}_{Y X}\left(\mathbf{R}_{n}, \tau \geq 0\right)$ for each different extracellular signal (see Section 2.3.2 for details). The input spikes are binned per time-step, and the spikes for the last $L$ time-steps are stored in a circular buffer $x$ of length $L$. During the course of the simulation, at every time-step, the binned input spikes in $x$ are multiplied with filter coefficients in vector $h$ and summed according to Equation (40). The index to vector $x$ is also adjusted such that the appropriate element of the circular buffer is accessed. The resulting filter output is stored in a (scalar) state variable, $y$, which can be recorded using a multimeter in NEST.

\subsection{Data availability and replicability}

\subsubsection{Codes and software tools}

This study has been made possible using the following software tools: Ubuntu 20.04.3 LTS (Focal Fossa), GCC 9.3.0, Python 3.8.10, ipython 7.13.0, jupyter-notebook 6.0.3, numpy 1.17.4, scipy 1.3.3, matplotlib 3.1.2, pandas 0.25.3, pymoo 0.4.2.2, mpich 3.3.2, mpi4py 3.0.3, h5py 2.10.0, NEURON 8.0.2, MEAutility 1.5.0, LFPykit 0.4, LFPy 2.2.4, 
LFPykernels 0.1.rc7 (https://github.com/LFPy/LFPykernels, git SHA: 62d15fb), NEST 3.1 (https://github.com/nest/nestsimulator, git SHA: 512022e), NESTML 4.0 (https://github.com/nest/nestml, git SHA: a3alb0d), parameters 0.2.1 (https://github.com/NeuralEnsemble/parameters, git SHA:b95bac2).

In order to ensure Methods and results reproducibility (Goodman et al. 2016; Plesser 2018), all simulation codes required to replicate the findings reported here will be available at https://github.com/LFPy/LFPykernels. These include the reference implementation of the methodology which is installable via the usual Python distribution channels as:

$$
\begin{aligned}
& \text { - pip install --pre lfpykernels \# or } \\
& \text { - pip install git+https://github.com/LFPy/LFPykernels }
\end{aligned}
$$

The code repository also includes a Docker recipe file which may be used to build containers with the full software environment required by the simulations and analysis.

Versioned releases of the LFPykernels tool is permanently deposited on https://zenodo.org (Hagen 2021)

\subsubsection{Hardware details}

All computationally demanding simulations for recurrent networks and reconstructed networks of MC neurons as well as parameter optimizations were performed on the standard compute nodes of the JUSUF compute cluster at the Jülich Supercomputing Centre (JSC), Jülich Research Centre, Jülich, Germany. Each compute node has two AMD EPYC 7742 CPUs $(2 \times 64$ physical cores) running at $2.25 \mathrm{GHz}, 256 \mathrm{~GB}$ of DDR4 RAM running at $3200 \mathrm{MHz}$. The compute nodes are interconnected by InfiniBand HDR100 (Connect-X6). Each MC network simulation ran in parallel distributed across 8 compute nodes with 1024 Message Passing Interface (MPI) processes, using the ParTec ParaStation MPI implementation. Point-neuron network simulations were executed using 32 OpenMP threads, 1 core per thread. All relevant softwares were compiled with compilers from GCC (9.3.0).

Post-processing, calculations of deterministic kernels, other analysis and plotting were performed on a MacBook Pro (13-inch, M1, 2020) with 16 GB RAM running macOS Big Sur (v11.6).

\section{Results}

\subsection{Neuron models with linearized dynamics}

The results presented throughout this study rely on three different fully active multicompartment (MC) neuron models, and versions where their voltage-dependent ion channel dynamics are linearized around a chosen membrane voltage value. These linearization steps are detailed in Section 2.2.2 pts. 2-3. The cell morphologies are shown in Figure 2A. The 'ball-and-sticks' models ' $\mathrm{E}$ ' and ' $\mathrm{I}$ ' represent excitatory and inhibitory neurons in the two-population recurrent network in Sections 3.2-3.7, while the biophysically detailed layer 5 pyramidal cell model (Hay et al. 2011) replaces the ball-and-sticks 'E' population in Section 3.8. The phenomenological 'E' and 'I' neurons are both modeled with a single compartment for the soma, and dendritic sections pointing up- and downwards along the depth-axis. The 'E' cell has a prominent apical section $1 \mathrm{~mm}$ in length while the 'I' cell dendritic sections are symmetric around the soma.

As a first check we stimulate the different cell models with small step-like input currents to the somatic compartment with negative and positive signs for $100 \mathrm{~ms}$ durations followed by a noisy current sequence (Figure 2B). In panels C-D we compare responses of the ' $E$ ' and 'I' model versions with 'quasi-linearized' versions of the $\mathrm{I}_{\mathrm{h}}$-type channel (biophys:lin) plus frozen dynamics for the $\mathrm{Na}_{\mathrm{t}}$ - and $\mathrm{SK}_{\mathrm{v} 1.3}$ channels as well as the version were also the $\mathrm{I}_{\mathrm{h}}$-type channel is frozen (biophys: frozen). In both cases the quasi-linearized versions are capable of capturing the subthreshold dynamics, including the sag and rebound effects which are explained by the $\mathrm{I}_{\mathrm{h}}$-channel currents. The fully passive-frozen models are effectively similar to models with only passive leak channels, which is reflected in the corresponding responses. These models then perform worse with respect to capturing the sub-threshold dynamics of the fully active versions. Note also that these linearized model neurons can not generate APs for stronger depolarizing input currents, unlike their active counterparts.

In Figure 2E, the same experiment is performed with the Hay et al. 2011 model neuron. Here, a quasi-linearized version of the Nap conductance is incorporated in addition to the $\mathrm{I}_{\mathrm{h}}$ channel, while remaining channels are in their passive-frozen states (biophys:lin). Again, the sub-threshold dynamics for small perturbations are captured by the quasi-linear model in an excellent manner, resulting in near identical response to that of the fully active model. Similar to our earlier observation, the model version with all passive-frozen dynamics (biophys: frozen) can not capture the somatic response accurately. 


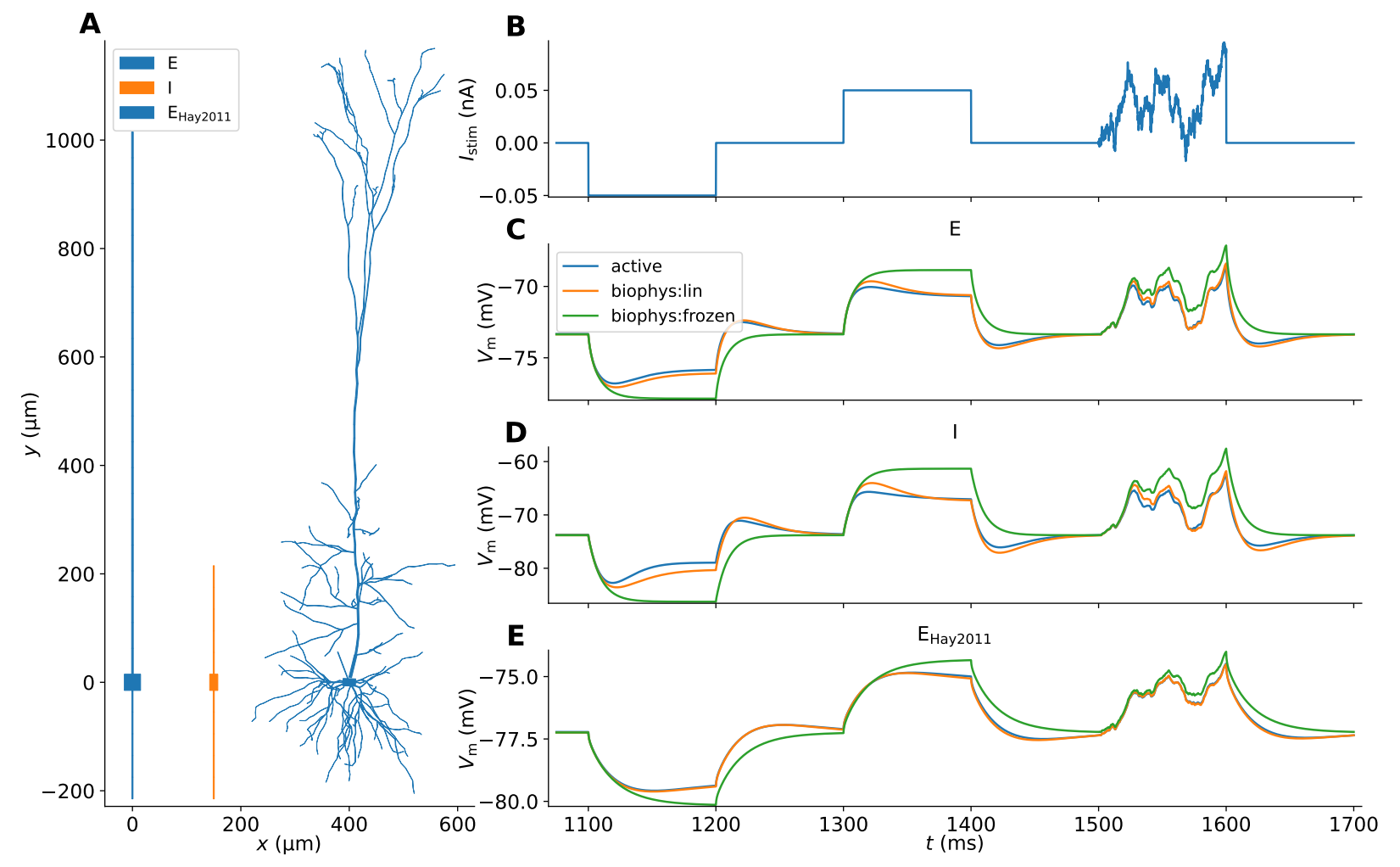

Figure 2: Model neurons and somatic responses with active and linearized ion-channel dynamics. (A) Neuronal geometries of neurons representing excitatory (E) and inhibitory (I) neurons, as well as a biophysically detailed pyramidal cell model replacing population ' $\mathrm{E}$ ' in the modified network. (B) Step input and noisy current stimulus delivered to the soma section of the three different cell modes. (C-E) Somatic responses to current input for the three cell models, incorporating their full set of active ion channels (active), linearized $\mathrm{I}_{\mathrm{h}}$-current (and $\mathrm{Na}$-current in case of the biophysically detailed neuron) dynamics with remaining channels in passive-frozen states (biophys:lin), and with all channels in their passive-frozen states (biophys : frozen).

\subsection{Reference MC neuron network with extracellular signal predictions}

In Figure 3A we show the populations of ball-and-sticks neurons and extracellular recording geometry for a phenomenological two-population MC neuron network set up according to pts. 1-13 in Section 2.1. For this network we predict extracellular potentials at depths highlighted by black circular markers treating compartments as line-sources (Equation (32)), as well as the current dipole moment (Equation (33)). The current dipole moment determines EEG and MEG like signals, as both can be computed from it using the appropriate forward model (Hämäläinen et al. 1993; Nunez and Srinivasan 2006; Hagen et al. 2018; Næss et al. 2021). Panels B and C show the distributions across depth of somas and instantiated synapses for each pair of pre- and postsynaptic populations, accordingly. A few somatic membrane potential traces recorded in each population is shown in panel $\mathrm{D}$. The median values $\left\langle V_{\mathrm{m}}(t)\right\rangle$ for a sample size of $N=1024$ in each population is used for linearization of ion-channel and synapse dynamics in the following sections. The spike raster plot (Figure 3E) shows the resulting activity to be stable and asynchronous-irregular at biologically plausible rates. The I cells fire more often than the E cells on average, around $5.1 \mathrm{~Hz}$ and $2.6 \mathrm{~Hz}$ respectively. Oscillations at the level of the populations are clearly visible in the corresponding spike-count histograms (panel F). These oscillations can be expected to be expressed in extracellular signals, and indeed the extracellular potential (panel G) shows oscillations with varying amplitudes across depth. We note in passing that the generated extracellular potentials are in line with experimentally observed signals with amplitudes of a few $100 \mu \mathrm{V}$, with few visible extracellular spike signatures. The oscillations generated by the network are prominently captured also in the current dipole moment (panel $\mathrm{H}$ ), however only in the vertical $z$-component $P_{z}$. Due to symmetry of the neural populations around the $z$-axis and the cell alignments along the same axis, the orthogonal components $P_{x}$ and $P_{y}$ cancel. Next, we investigate how these signals may be captured by models that only use MC neurons with linearized ion-channel and synapse dynamics, and no recurrent connections. 

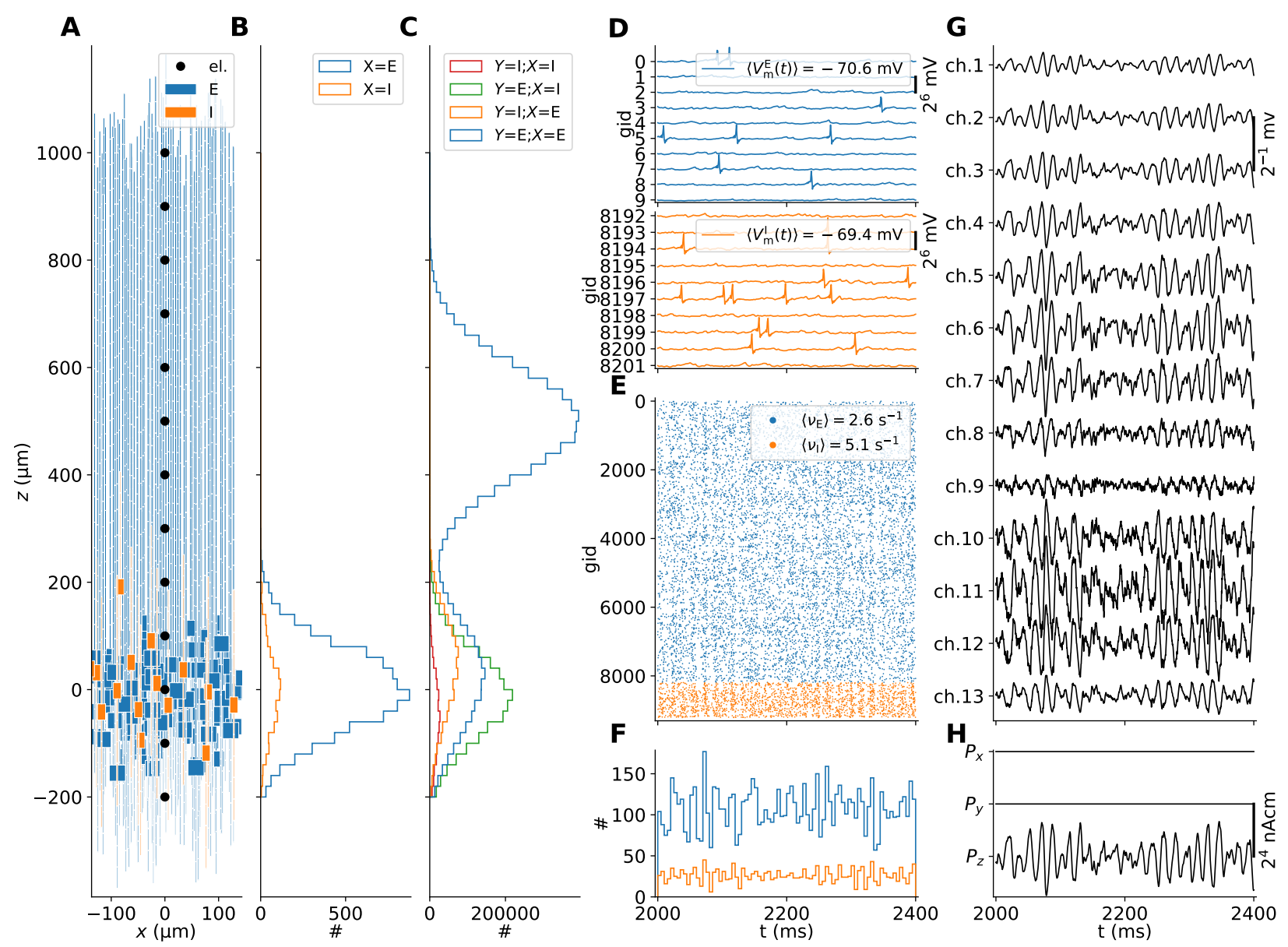

Figure 3: Stylized two-population MC neuron network with ground truth predictions of extracellular signals. (A) Neuronal populations and electrode geometry. The network is constructed of one excitatory (' $E$ ') and one inhibitory ('I') population. Only a subset of cells are shown from each population. The black point markers along the $z$-axis denote locations of electrode contact points with separation $100 \mu \mathrm{m}$. (B) Soma counts per population $X$ along the vertical $z$-axis in bins of $20 \mu \mathrm{m}$. (C) Synapse counts per connection $K_{Y X}$ along the vertical $z$-axis (bin size $20 \mu \mathrm{m}$ ). (D) Somatic potential traces of 10 neurons in populations ' $E$ ' and ' $I$ '. The $\left\langle V_{\mathrm{m}}(t)\right\rangle$ values in each legend denote median soma potentials computed from a subset of neurons in each population $(N=1024)$. (E) Network spike raster spanning $500 \mathrm{~ms}$ of spontaneous activity. The mean population-averaged firing rates are given shown in the legend. (F) Per-population spike-count histograms with bin size $5 \mathrm{~ms}$. (G) Extracellular potentials. (H) Components of the current dipole moment along the $x, y, z$-axes. 


\subsection{Hybrid model with linear dynamics accurately captures extracellular signals of the reference network}

Biophysically detailed as well as simplified networks of spiking point-neuron models can generate realistic spike train statistics of different populations. But, the presently used framework combining MC and VC models is required in order to compute meaningful extracellular population signals such as the LFP. In the hybrid scheme (Hagen et al. 2016; Section 2.2), the simulation of spiking activity from recurrent network(s) can be performed separately with intermediate storage of spikes, while extracellular signals can be computed via unconnected populations of MC neurons activated by synapses triggered at times as they would have occurred in the actual network. Using the recurrent MC neuron network and corresponding signals we can now, in contrast to our earlier study (Hagen et al. 2016), test this prediction scheme in a self-consistent manner.

In this test we record spikes trains of each neuron and ground truth extracellular potentials and current dipole moment from our reference MC neuron network to file, as well as the randomly instantiated cell locations in space and the full synaptic connectivity. The resulting connectivity table includes pre- and postsynaptic neuron id, synaptic location (cell morphology coordinate and Cartesian coordinate), maximum synaptic conductance and transmission delay. Locations and activation times of extrinsic synapses are not stored directly due to their large count, only random seeds affecting these, ensuring replicability on the same compute resources.

With the above information, we confirmed we can compute the intra- and extracellular signals matching the ground truth exactly, as initial conditions, neuron models and synaptic activations etc., can be preserved in absence of actual recurrent connections (result not shown). However, one benefit of the present hybrid scheme, is that it allows simplifying the individual neuron and synaptic dynamics systematically. In particular we shall test the idea that a linearized model setup can accurately capture the spatiotemporal features of the ground truth extracellular potential $\left(V_{\mathrm{e}}(\mathbf{R}, t)\right)$ and current dipole moment $(\mathbf{P}(t))$ signals.

We first consider 4 hybrid model configurations. These configurations all incorporate the same linear approximation to synaptic currents around the median somatic voltage in each network population as described in pt. 1 in Section 2.2.2. Then, we consider every possible permutation of (1) whether or not to account for changes in the effective membrane leak conductance $G_{\text {eff }}$ per compartment $m$ due to synaptic activity (see pt. 5 in Section 2.2.2), and (2) the quasilinearized (biophys:lin) and passive-frozen (biophys:frozen) model neuron variants representative of the 'E' and 'I' population showcased in Section 3.1. See pt. 2-3 in Section 2.2.2 for details on the linearization procedure for voltage-gated ion channel descriptions.

By visual inspection of all 'hybrid scheme' predictions in Figure 4, we see that both model setups accounting for changes in the effective membrane leak conductance ( $g$ _eff :True) in panel B accurately captures the spatiotemporal features of the ground truth signals (black lines), including signal amplitudes. The main differences seen here are that the ground truth signals contain high frequency jitter that is not captured in hybrid scheme predictions as signal contributions by APs are not accounted for. By visual inspection, our choice of quasi-linearized (biophys: lin) or passive-frozen (biophys:frozen) ion-channel dynamics are seen to have remarkable little effect on the predicted signals (in contrast to somatic voltage responses). The corresponding curves fully overlap with each other in the plot. However, not accounting for membrane conductance contributions by synapses (g_eff:False), results in a clearly detrimental effect on the predicted signals (panel A). The most salient observation is that the approximations to extracellular potentials across depth $V_{\mathrm{e}}(\mathbf{R}, t)$ as well as $z$-components of the current dipole moment $\left(P_{z}(t)\right)$ are predicted with amplitudes that are about a factor 2 too high. The signals also appear to lag behind the ground truth signals by a few $\mathrm{ms}$ in the temporal domain. These effects are also observed in a preliminary report on this particular hybrid scheme model configuration (Magnusson 2021). A more thorough analysis and summary of the accuracy of these signal approximations seen are summarized in Section 3.5, and compared also to kernel based prediction methods. For the remainder of this study we will assume that signal approximation methods other than the hybrid scheme must also account for changes in the leaky properties of the membrane, due to the effect the (effective) membrane time constant has on the integration of synaptic input currents throughout the dendrites and resulting distributions of transmembrane currents.

\subsection{Kernels for accurate signal predictions}

So far we have showed that 'hybrid scheme' models incorporating linear approximations to the synapse and active ion channel currents accurately capture the extracellular potentials across depth as well as the current dipole moment. This observation implies that the relations between times of presynaptic APs and resulting spatiotemporal distribution of transmembrane currents (and therefore extracellular potentials etc.) of respective postsynaptic neurons are approximately fixed. As the postsynaptic responses can not occur before the spike times of presynaptic neurons, these relationships must also be causal. Throughout this and the next sections we shall therefore further test the idea that extracellular signal predictions can be well represented as a linear time-invariant (LTI) causal system. Here, we shall compare 
A
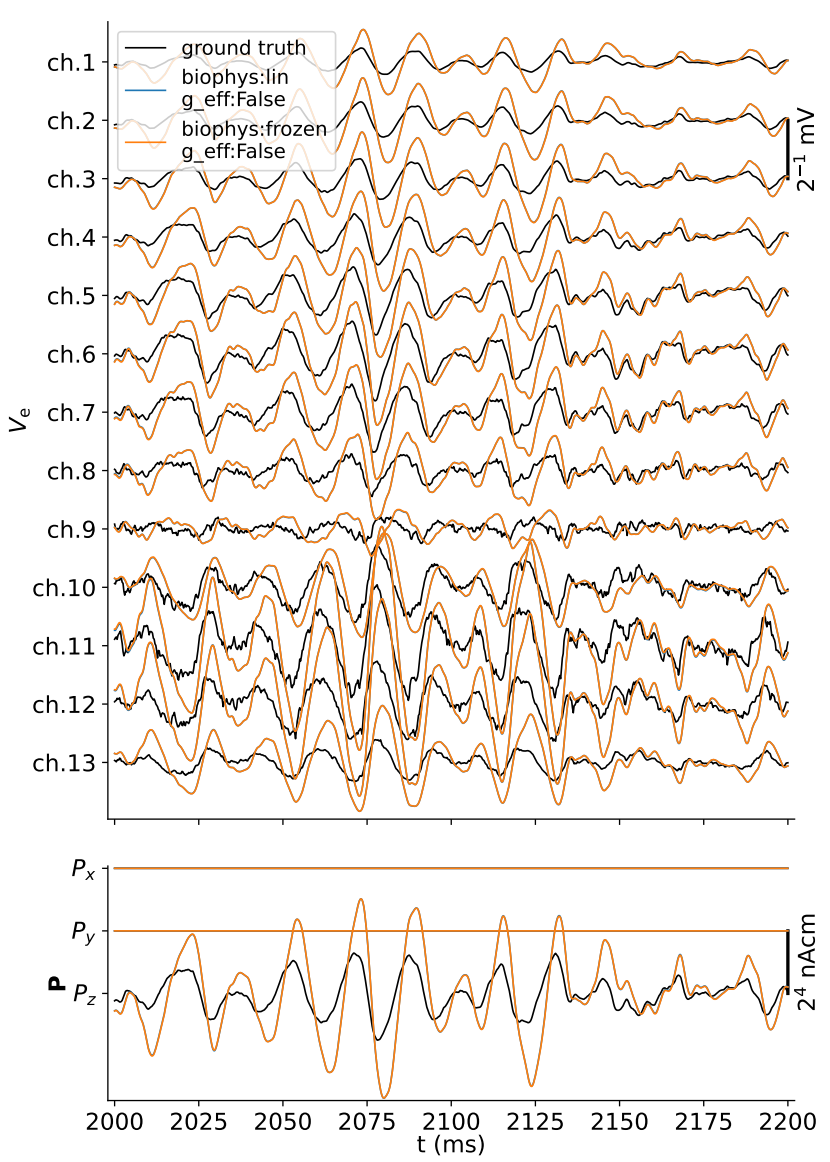

B
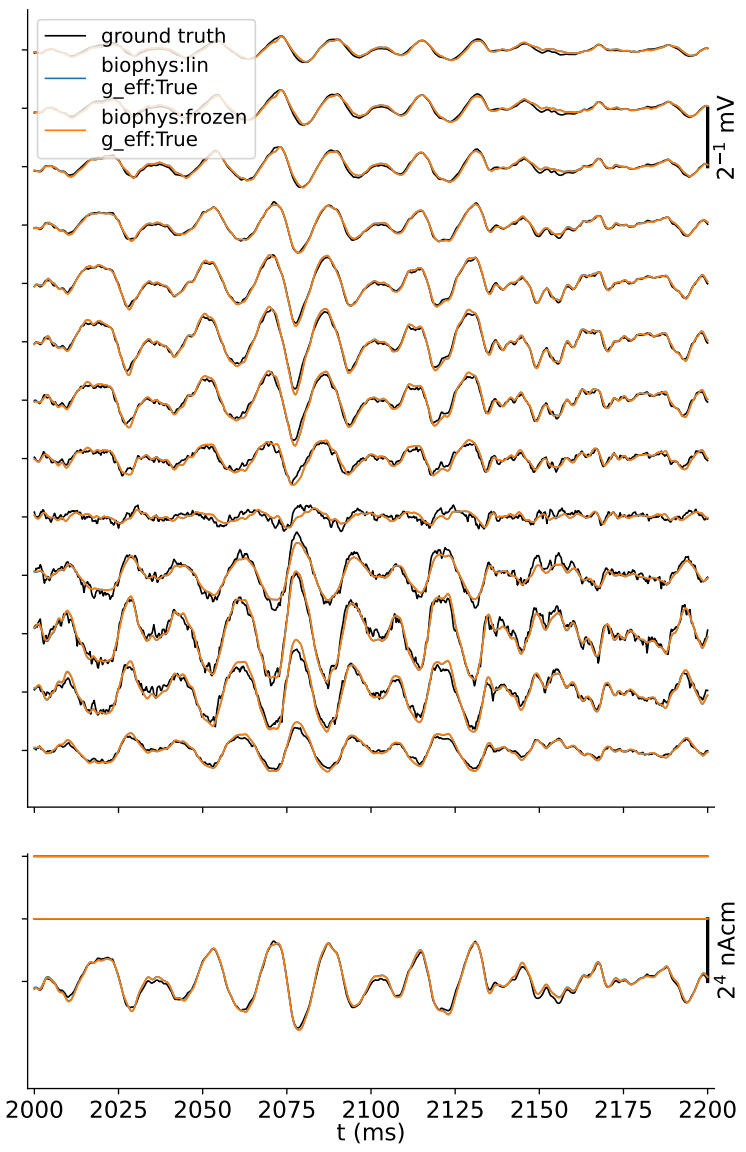

Figure 4: Ground truth signals vs. hybrid scheme approximations. Extracellular potential across depth (top row) and current dipole moment (bottom row) predicted from a MC neuron network model (black lines) is compared to predictions made using the hybrid scheme, with either passive-frozen or quasi-linear MC neurons (biophys : frozen vs. biophys:lin), ignoring or accounting for the effective membrane conductance (g_eff:False/True in panels A and $B$ respectively).

filter coefficients, or 'kernels', obtained at the population level using two different approaches, either via the 'hybrid scheme' setup above, or using a novel, deterministic method based on the idea that the underlying distributions of cell and synapse positions, synaptic delays as well as linearized ion-channel and synapse dynamics provide sufficient information to estimate the corresponding causal filters. Our derivation of these deterministic kernels are described in detail in section 2.3.2. In both cases the kernels represent the population-averaged postsynaptic response of spike events in each presynaptic population, that is, equivalent to 'spike-signal' impulse response functions of the system. For corresponding signal predictions evaluated in Section 3.5, the kernels are applied with population spike rates. Predictions are there compared with ground truth signals generated by our reference recurrent MC neuron network (see Section 3.2).

\subsubsection{Predicted kernels using the hybrid method}

Outlined in Section 2.3.1, we could potentially estimate the full set of kernels for every connected pair of pre- and postsynaptic neurons and compute the signal using Equation (21). However, this approach is intractable in large networks due to the connection count and corresponding kernel count. Hagen et al. 2016 already showed that averaged kernels $H_{Y X}(\mathbf{R}, \tau)$ computed for presynaptic $(X)$ and postsynaptic $(Y)$ populations could accurately capture the corresponding hybrid scheme extracellular potentials by the double sum over population firing rates convolved by averaged kernels (see Equation (25)). 

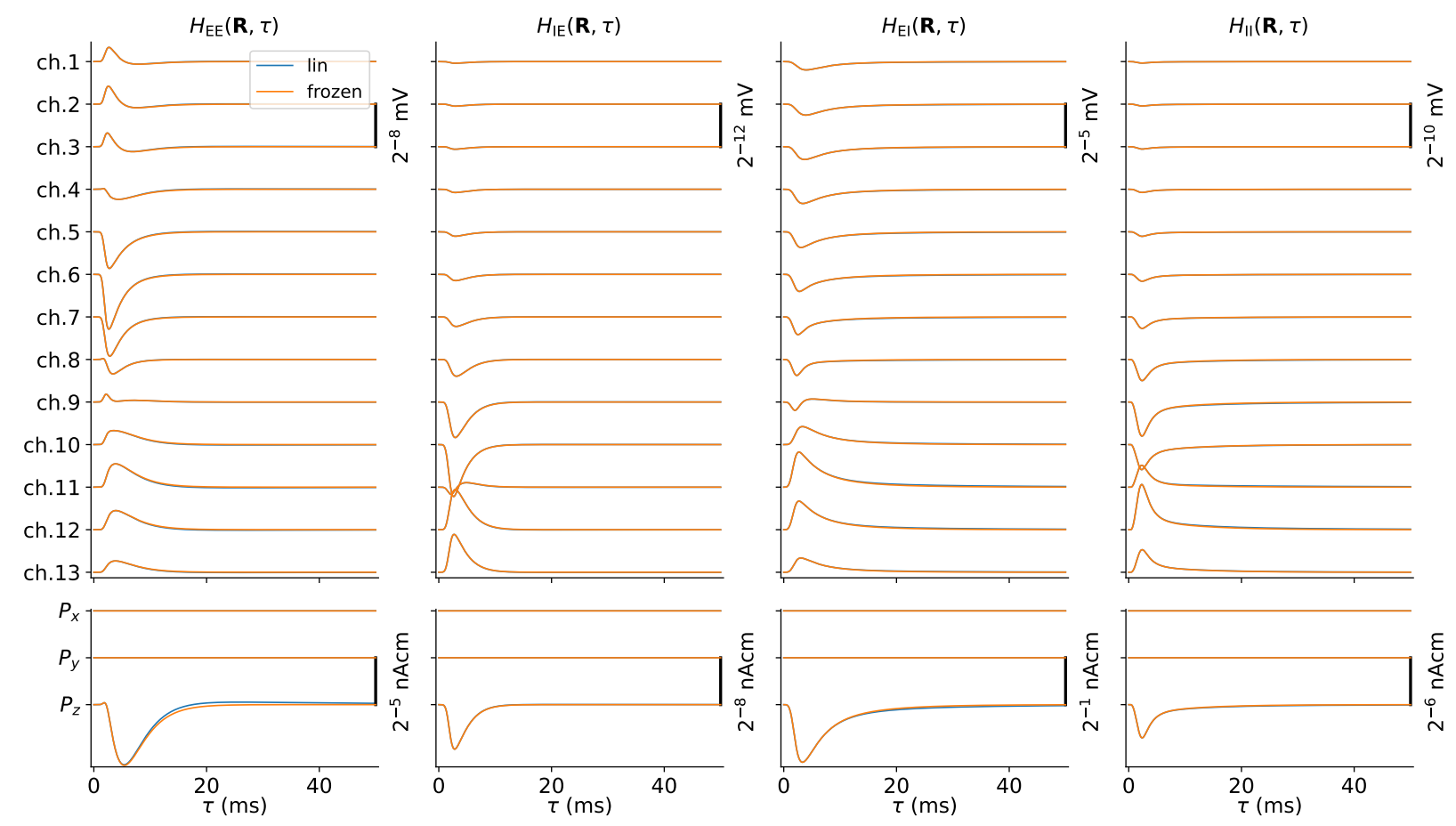

First, we adapt the hybrid scheme simulation above, using current-based synapses and either variants of linearized ion-channel dynamics. We account for changes in the effective membrane leak conductance as above. Then, ongoing spiking activity in each population is replaced by single synchronous events that allows for computing the full set of population-averaged kernels $H_{Y X}(\mathbf{R}, \tau)$ using Equation (22). The resulting sets of kernels for predicting the extracellular potential and current dipole moment are shown in Figure 5. Consistent with our earlier observation, only minor differences occur between signals predicted using quasi-linear or passive-frozen cable models. The set of kernels reveal non-trivial relationships between spikes by neurons in each population and the extracellular potential across depth due to combined effects of the cable models, synapse model, VC model, etc., and could challenge model assumptions made in other studies like space- and time-separable kernels (e.g., Einevoll et al. 2007; Głąbska et al. 2016; Mazzoni et al. 2015) due to the effect of dendritic integration.

The set of kernels also allow for some insight in which connections and populations shape the extracellular signals. Here, the I to E kernel $\left(H_{\mathrm{EI}}(\mathbf{R}, \tau)\right)$ has amplitudes that are $\sim 4-16$ times those of the $\mathrm{E}$ to $\mathrm{E}$ kernel $\left(H_{\mathrm{EE}}(\mathbf{R}, \tau)\right)$. Thus any spike in population 'I' may give a significant signal contribution from inhibitory synaptic currents in population 'E', even if the number of neurons in population ' $E$ ' is 8-fold that of population 'I'. Dominance of inhibitory over excitatory contributions in the LFP is in agreement with previous reports (e.g., Hagen et al. 2016; Teleńczuk et al. 2017). Furthermore, the direct contribution by spike-evoked transmembrane currents on population 'I' can be expected to be minor, in part explained by the smaller spatial extents of the neurons and low cell count.

Figure 5: Hybrid scheme spike-LFP and spike-current-dipole-moment impulse response functions ('kernels'). Spatiotemporal functions $H_{Y X}(\mathbf{R}, \tau)$ for each connection between every possible pre- and postsynaptic network population $X$ and $Y$, respectively. The top row kernels are computed as the spike-averaged contribution by postsynaptic neurons to the extracellular potential in electrode contact locations shown in Figure 3A, while the bottom row kernels are computed as the spike-averaged current-dipole moment contribution along the horizontal $(x, y)$ and vertical $z$-axis. The kernels are computed either using fully passive-frozen (biophys : frozen) or with quasi-linearized (biophys: 1 in) cable models. The kernels are truncated at time lags $\tau \in[0,50]$.

\subsubsection{Deterministic kernel predictions}

The kernel calculations via the hybrid scheme above relies on a number of MC neuron simulations proportional to the overall network size, and incur at significant computational costs. Here we rather account for distributions and expectation values in the parameterization of the MC neuron network directly, allowing predictions of an appropriate and accurate set of kernels without instantiating network populations of MC neurons. Illustrated in Figure 6 and described fully in Section 2.3.2, the constituents needed are: (panel A) linearized versions of the MC neurons representing each 
population; (panel B) their distribution in space; (panel C) probabilities for synaptic placements per compartment for each main connection; (panel D) synaptic indegree distributions over instantiated connections for each main connection; (panel E) conduction delay distribution for each main connection and finally (panel F) the linearized synapse currents for each main connection. In addition, the VC model for each signal is modified to account for radially symmetric cell distributions in space (see Section 2.4.2 for details). Also typical presynaptic spike rates needs to be specified as well as pairwise connection probabilities between neurons in each population.

In contrast to the above 'hybrid scheme' kernels shown in Figure 5, each kernel now requires only a single MC neuron simulation to compute the averaged transmembrane currents following synaptic activation, and account for all other effects by a series of linear convolution operations in the spatial and temporal domains as well as a scaling by the presynaptic population size (see Section 2.3.2 for details). The set of calculations results in deterministic outcomes, and are fast to compute on laptop computers while High-Performance Computing (HPC) resources are generally required for the 'hybrid scheme' setup. From our default parameterization of the MC neuron network (Section 2.1), the resulting set of approximated kernels $\hat{H}_{Y X}(\mathbf{R}, \tau)$ for each main connections between pre- and postsynaptic populations $X$ and $Y$ is shown in Figure 7. This new set of kernels appear very similar to the averaged kernels computed via the hybrid scheme shown in Figure 5, suggesting that they may be used interchangeably. Next, we shall apply our predicted kernels with corresponding population spike count histograms ('spike rates') for signal approximations, and compare their accuracies alongside predictions using the full hybrid scheme (Section 3.3).
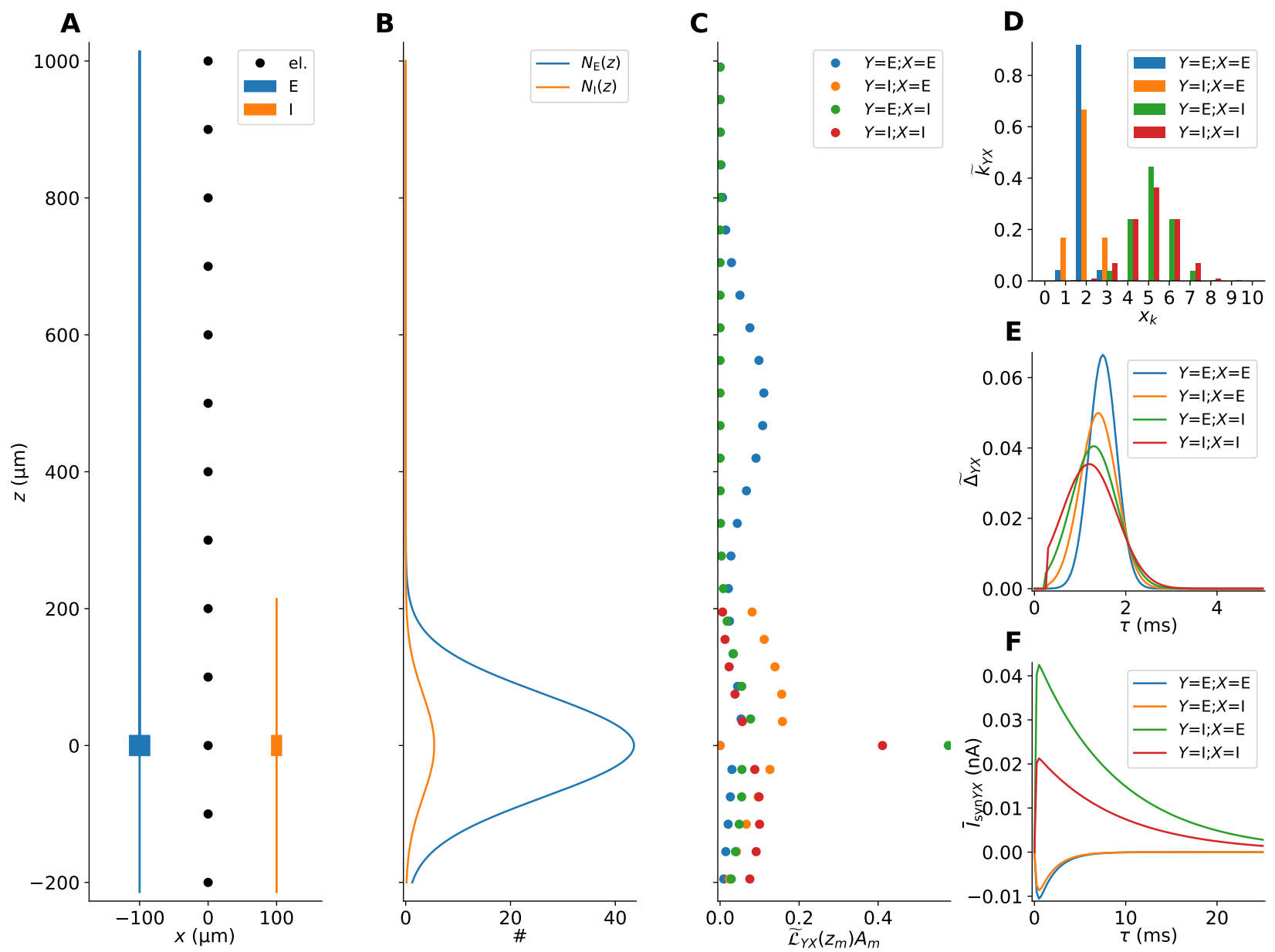

Figure 6: Components for predictor of spike-signal filter kernels $\hat{H}_{Y X}(\mathbf{R}, \tau)$. (A) MC neuron models representative of each population 'E' and 'I', with layout of extracellular laminar probe. (B) Densities of somas along $z$-axis. (C) Per-compartment synapse connection probability for each pathway computed as the product of depth dependencies and compartment surface areas. (D) Probability mass functions of multapse distributions for instantiated connections. (E) Normalized distributions of transmission delays $\widetilde{\Delta}_{Y X}(\tau)$ for each connection relative to presynaptic activation time. (F) Linearized synaptic currents $I_{\operatorname{syn} Y X}(\tau)$ relative to synaptic onset for each connection. 


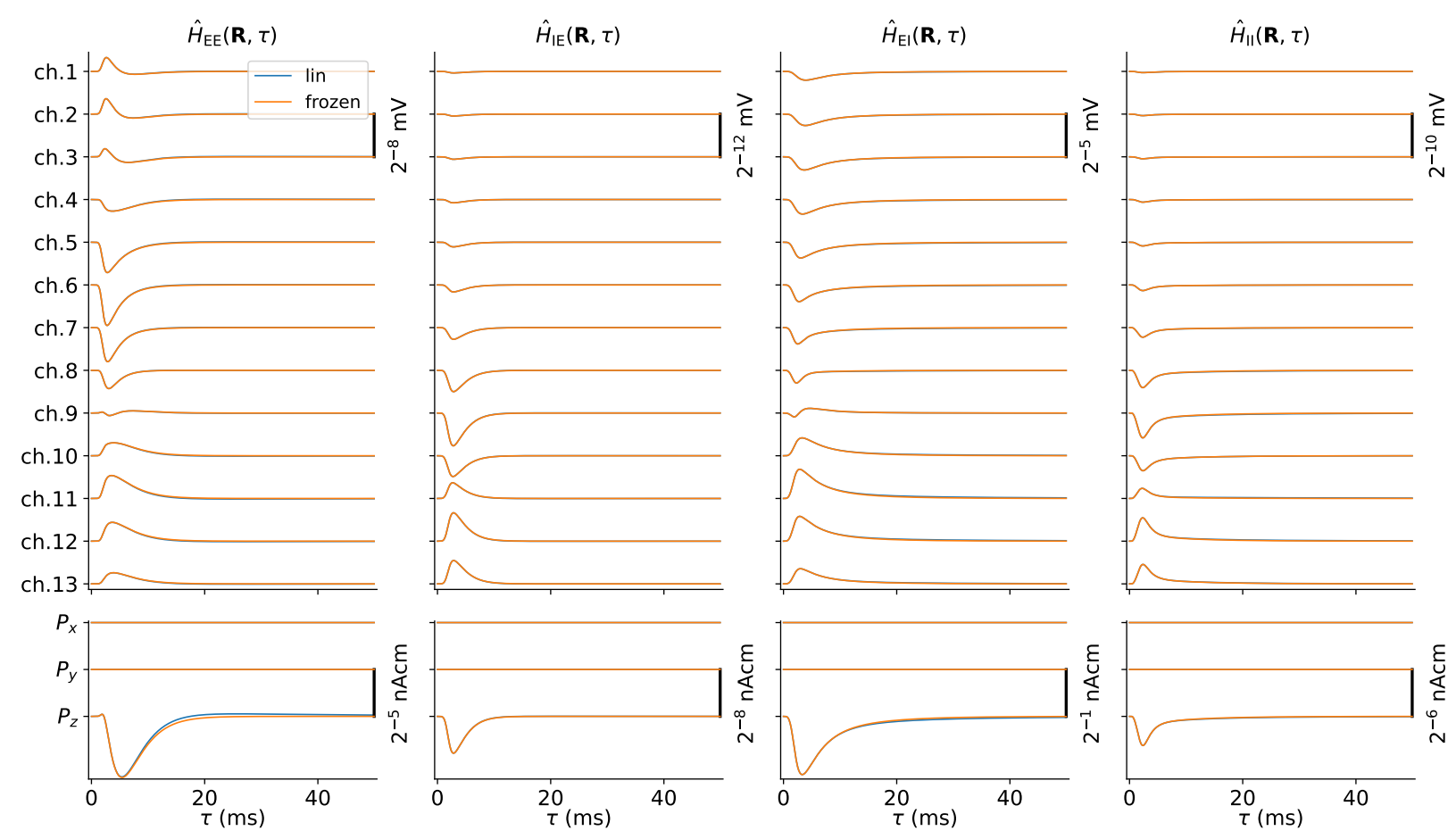

Figure 7: Spike-LFP impulse response function predictions. Similar to Figure 5, but here computed using a computationally fast and deterministic method accounting for expectation values in terms of cell and synapse placement.

\subsection{Accurate signal predictions using hybrid scheme and deterministic kernels}

With the sets of hybrid scheme kernels $\left(H_{Y X}(\mathbf{R}, \tau)\right.$ in Figure 5) and approximated kernels $\left(\hat{H}_{Y X}(\mathbf{R}, \tau)\right.$ in Figure 7) we now convolve them with the corresponding presynaptic population firing rates $\nu_{X}(t)$, and sum up the contributions using Equation (25). In all respects, the corresponding signal predictions shown in Figure 8 panels A and B compare very favorably with the ground truth signals. Both the extracellular potential and current dipole moment signals closely matches the ground truth in terms of spatiotemporal features and signal amplitudes.

By visual inspection, neither hybrid scheme predictions (Section 3.3, Figure 4) nor kernel-based predictions display clearly distinguishable discrepancies from the ground truth signals, except for some high-frequency jitter associated with APs present in the ground truth data. In order to quantify prediction accuracies we therefore resort to comparing squared Pearson correlation coefficients $\left(R^{2}\right.$, Equation (38)) between ground truth signals and predictions, as well as relative differences in their standard deviations $\left(r_{\mathrm{STD}}\right.$, Equation (37)). We compute these metrics not only for the 'raw' signals but also for low-pass ('LP') filtered data. Thus by attenuating the higher frequencies typically associated with presynaptic APs present in the ground truth (see Section 2.5 for details) a somewhat improved accuracy for the different approximations can be expected. In terms of extracellular potentials and the low-pass filtered counterpart (a.k.a. the local field potential - LFP), the $R^{2}$ and $r_{\text {STD }}$ metrics in Figure 9 confirm our visual analysis. The worst-performing configurations are hybrid scheme setups that do not account for changes in the effective membrane time constants (g_eff:False). All others perform well in all channels except Ch. 9. Nearby this depth, the sign of the signals flips due to current conservation, perhaps most evident in the dominating kernels $H_{E I}(\mathbf{R}, \tau)$ and $\hat{H}_{E I}(\mathbf{R}, \tau)$ shown in Figure 5 and 7. Except for the Ch. 9 outliers, the observed $R^{2}$ and $r_{\mathrm{STD}}$ values approach 1 . Figure 9 panels $\mathrm{C}$ and D projects median as well as the $10 \%$ and $90 \%$ percentiles of $R^{2}$ and $r_{\text {STD }}$ values computed across channels. Here, an overall gain in $R^{2}$ is seen in all cases in the low-pass filtered datas. Overall, our choice of quasi-linearized vs. passive-frozen membrane dynamics has only a minor effect in terms of the $r_{\mathrm{STD}}$ and $R^{2}$ metrics.

Finally, our findings for the extracellular potentials are mirrored for the approximated $z$-component of the current dipole moment in panels $\mathrm{E}$ and $\mathrm{F}$ in Figure 9. All approximations taking into account the effect of the effective membrane leak conductance performs excellently, both with respect to the $R^{2}$ and $r_{\mathrm{STD}}$ metrics. 


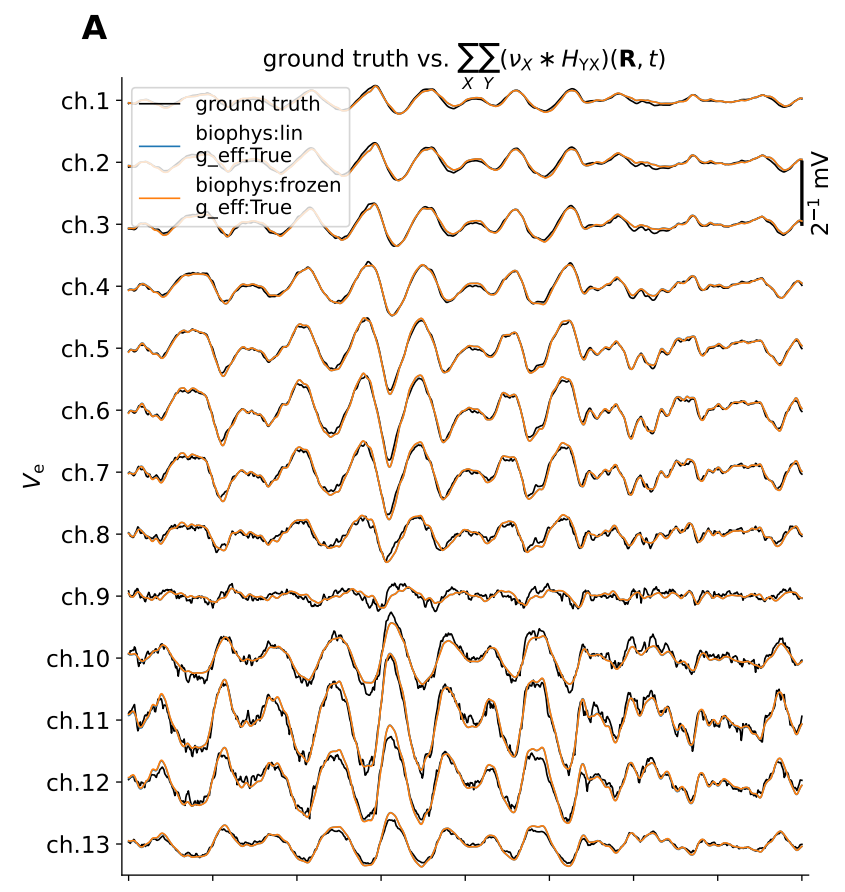

B
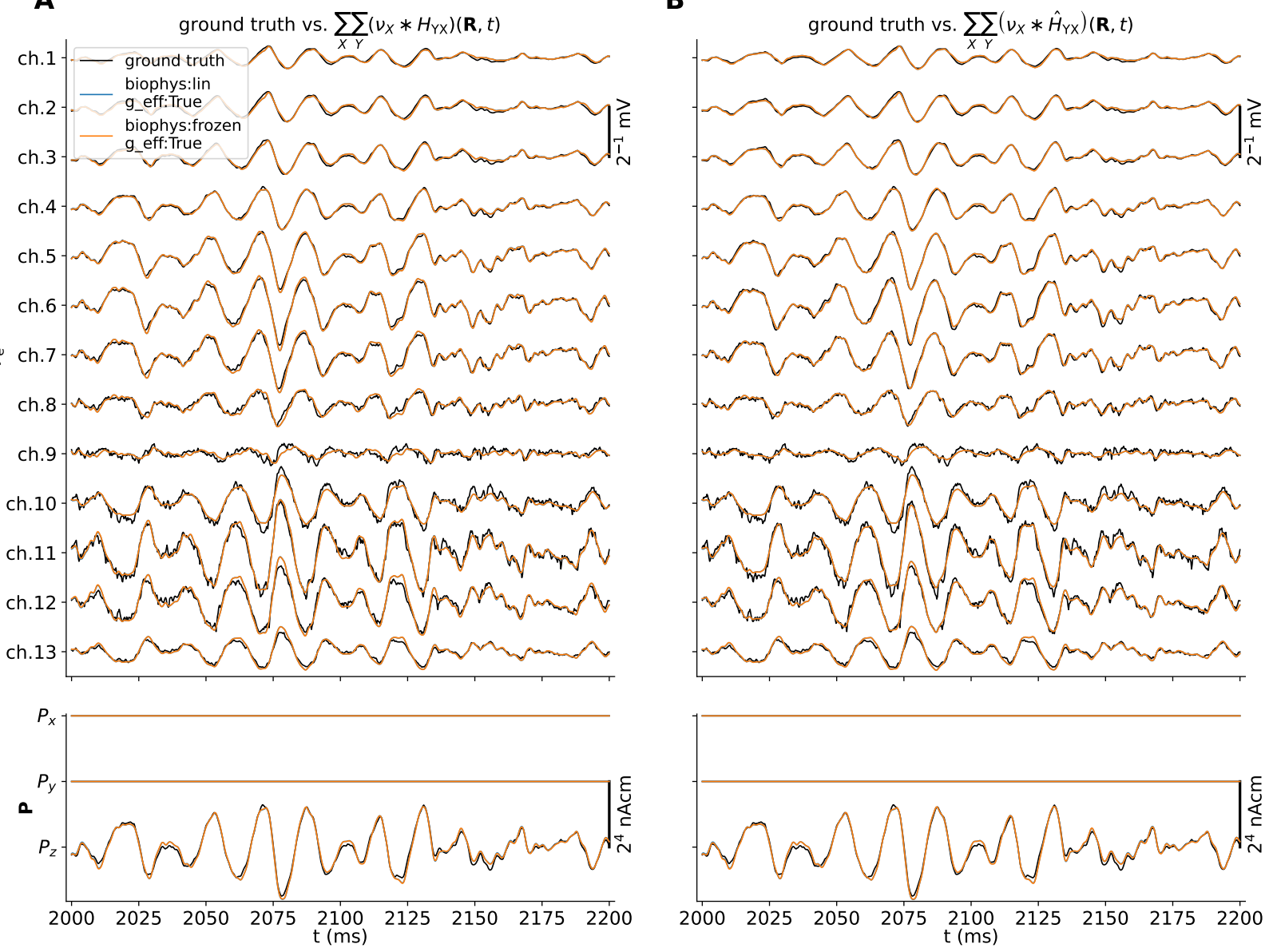

Figure 8: Ground truth signals vs. kernel-based approximations. (A) Ground truth extracellular potential (top) and current dipole moment (bottom) predicted from a MC neuron network model compared to predictions made using the hybrid scheme kernels $H_{Y X}(\mathbf{R}, \tau)$ shown in Figure 5. The kernels are measured using either passive-frozen or quasi-linear MC neurons (biophys: frozen vs. biophys: lin), accounting for the effective membrane conductance (g_eff: True). The signal approximations are obtained by convolving presynaptic population firing rates $\left(\nu_{X}(t)\right)$ with respective kernels $\left(H_{Y X}(\mathbf{R}, \tau)\right)$ and summing the contributions. (B) Same as panel A, using deterministic kernels $\hat{H}_{Y X}(\mathbf{R}, \tau)$ shown in Figure 7.

\subsection{Effect of perturbed parameters on signal predictions with deterministic kernels}

Predictions of $\hat{H}_{Y X}(\mathbf{R}, \tau)$ and corresponding signals rely on accurate assessments of a number of parameters. Here we choose to investigate the effect on $\hat{H}_{Y X}(\mathbf{R}, \tau)$ of mismatched time-averaged presynaptic population firing rates (including that of the external population) $\left\langle\nu_{X}(t)\right\rangle$ and choice of $\left\langle V_{\mathrm{m}}(t)\right\rangle$ on our $R^{2}$ and $r_{\mathrm{STD}}$ metrics. These statistics are computed for rate-based time-series predictions against corresponding ground truth datas. For brevity, we chose to compute these metrics only for the $z$-component of the current dipole moment $\left(P_{z}(t)\right)$. In results above this term appears to be a valid indicator for corresponding metrics computed from extracellular potentials $\left(V_{\mathrm{e}}(\mathbf{R}, t)\right)$. The parameter $\left\langle\nu_{X}(t)\right\rangle$ directly affects the calculation of the effective leak conductivity values $g_{\mathrm{eff} m}$ via Equation (28), while $\left\langle V_{\mathrm{m}}(t)\right\rangle$ affects the linearization steps applied to voltage-gated ion channels and synaptic currents as detailed in Section 2.2.2. For brevity, we compute kernels employing neuron models with passive-frozen ion-channel dynamics.

The contour lines denoting $R^{2}$ equal to $0.95,0.98$ and 0.99 in Figure 10A demonstrate that a a relatively broad range of parameter values results in good temporal agreement between the approximated and ground truth signals. When $\left\langle V_{\mathrm{m}}(t)\right\rangle$ is shifted by $-10 \mathrm{mV}$ the signal contributions by inhibitory synapses drop significantly as the difference to the inhibitory synapse reversal potential diminish. If the assumed presynaptic rates are all rescaled to zero (the ratio $\left\langle\nu_{X}^{\diamond}\right\rangle /\left\langle\nu_{X}\right\rangle=0$ ), it amounts to ignoring the effective leak conductivity altogether as $g_{\text {eff } m}=\bar{g}_{\mathrm{L}}$. A minor gain may be 

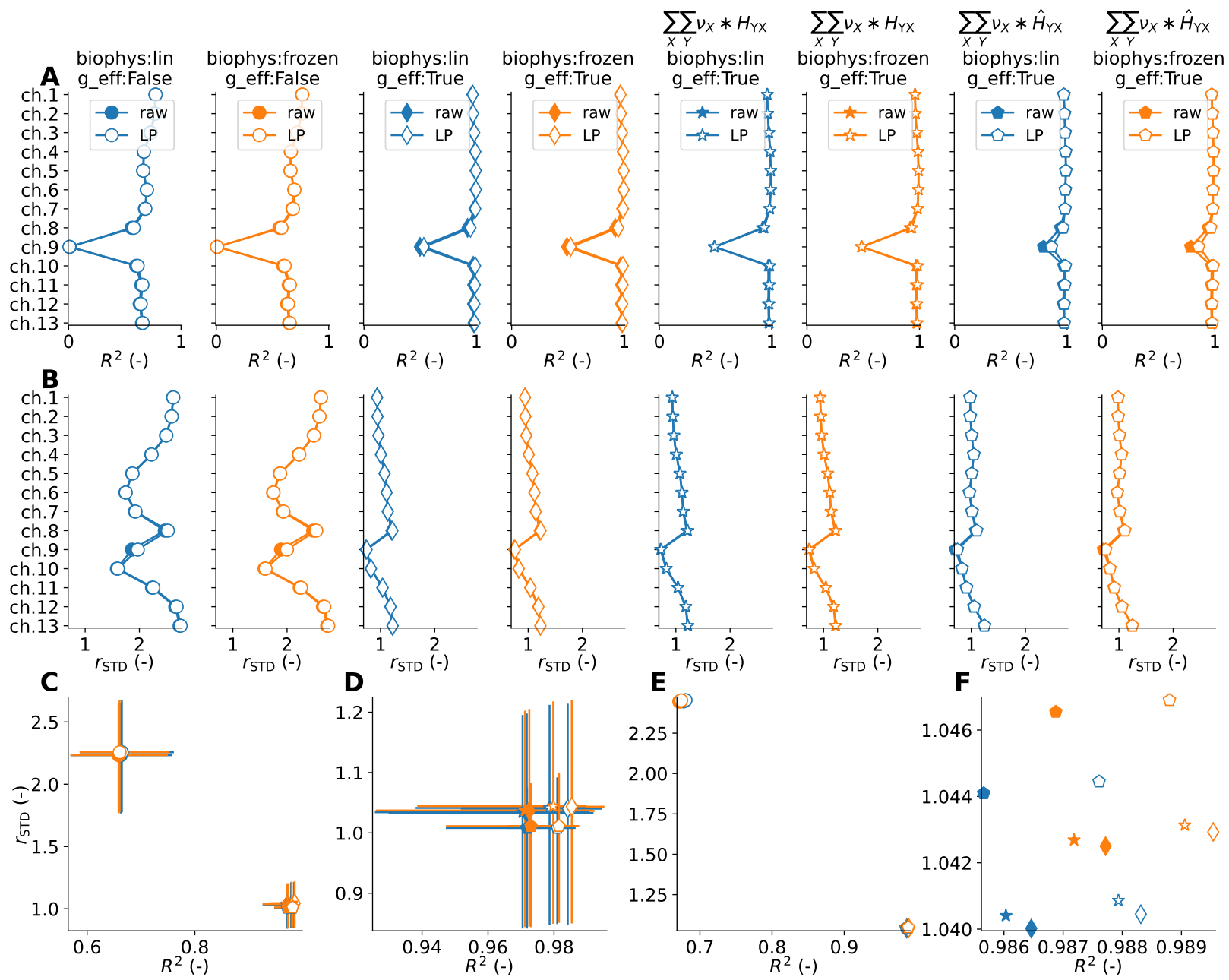

Figure 9: Accuracy of signal predictions vs. ground truth. For each signal approximation shown in Figure 4 and 8, their accuracy is evaluated in terms of the (A) squared Pearson correlation coefficient between approximation and ground truth $\left(R^{2}\right)$ and $(\mathbf{B})$ their standard deviation normalized by ground truth signal standard deviation $\left(r_{\mathrm{STD}}\right)$. The filled and white-faced markers denote metrics computed from raw and low-pass filtered data, respectively. (C) Aggregate $R^{2}$ and $r_{\mathrm{STD}}$ values with median, $10 \%$ and $90 \%$ percentiles along each axis computed from extracellular potential approximations. Outliers not shown. (D) Same as panel C for predictions accounting for changes in effective membrane conductance (g_eff: True). (E) Scatter plot of $R^{2}$ vs. $r_{\mathrm{STD}}$ for the different approximations to the $z$-component of the current dipole moment $\mathbf{P}(t)$. (F) Same as panel $\mathrm{E}$ for predictions accounting for changes in effective membrane conductance (g_eff : True). 
seen for low-pass filtered data. The $r_{\mathrm{STD}}$ values computed across the same parameter space in Figure 10B show a more gradual dependency on each parameter. Acceptable $r_{\mathrm{STD}}$ values occur along a diagonal line (contour line labeled '1.0').

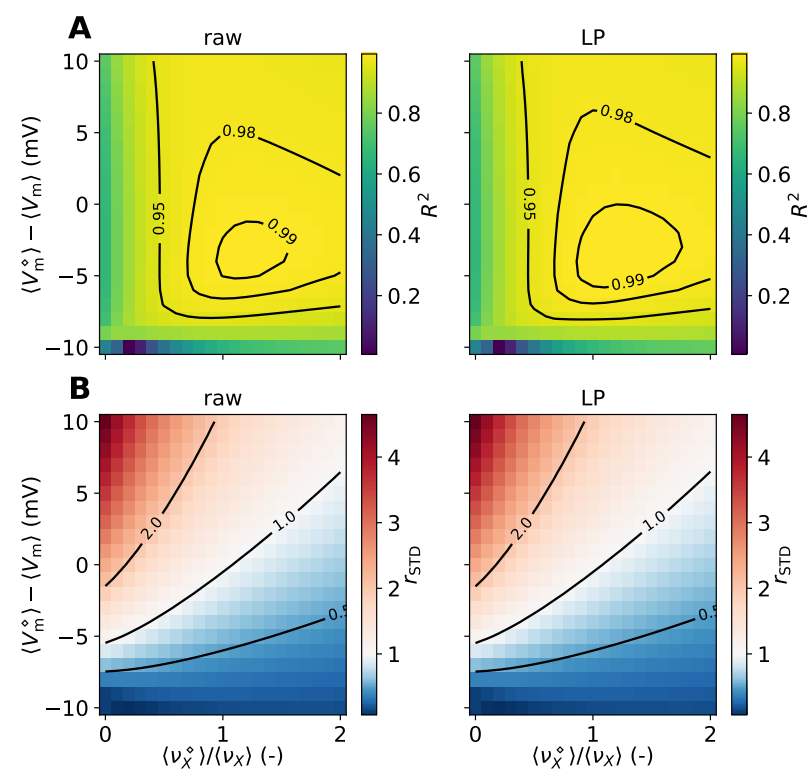

Figure 10: Effect of mismatched presynaptic firing rates and membrane potentials on kernel-based approximations to the current dipole moment signal. A Effect on the $R^{2}$ metric computed between ground truth $z$-component of the current dipole moment $\left(P_{z}(t)\right)$ and corresponding kernel-based approximations. For each datapoint in each panel, the kernel approximations $\hat{H}_{Y X}(\mathbf{R}, \tau)$ are computed when shifting the linearization membrane voltage by $\left\langle V_{\mathrm{m}}^{\diamond}\right\rangle-\left\langle V_{\mathrm{m}}\right\rangle$ and multiplying the presynaptic firing rates by a factor $\left\langle\nu_{X}^{\diamond}\right\rangle /\left\langle\nu_{X}\right\rangle$. The superscript $\diamond$ denotes perturbed values. The panels show $R^{2}$ computed for kernels assuming passive-frozen (biophys : frozen) ion-channel dynamics. The left and right columns show $R^{2}$ computed from raw and low-pass (LP) filtered data, respectively. B Same as panels in A but for $r_{\mathrm{STD}}$ metric.

\subsection{Methods performance for perturbed network states}

So far (in Sections 3.3 and 3.5), we have showed that fully linearized model setups can accurately approximate the ground truth extracellular signals of a recurrent MC neuron network. The main linearization tricks (detailed in Section 2.2.2) are (1) approximations of the conductance-based synapses by equivalent current-based synapses and (2) approximations of the active ion conductances by linearized versions. A crucial parameter in both cases is the choice of the postsynaptic membrane potential $\left\langle V_{\mathrm{m}}(t)\right\rangle$ which is assumed constant. Throughout this manuscript we have chosen the median somatic membrane potential averaged over neurons in each population of the recurrent networks. However, there are several scenarios where this assumption of near constant postsynaptic membrane potentials can be expected to fail. This may include the presence of large-conductance synapses where synapse activation may result in significant de- and hyper-polarizations of the postsynaptic membrane potential, as well as synchronous network states where the variance in membrane potentials may increase with increased strength of network oscillations.

As the accuracies seen for the different approximations are observed to be so similar, we here chose to assess the accuracy of kernel-based approximations for perturbed networks in terms of modified connectivity parameters, using sets of kernels estimated directly from neuron models using passive-frozen ion-channel dynamics. For this purpose, we rescale all recurrent synaptic connection weights in the recurrent MC neuron network by the same scaling factor $J$ (see Section 2.1.2), rerun network simulations in order to provide new ground truth data, spike trains and somatic potentials, and recompute the set of kernels for each $J$ value. The scaling factor affects both the degree of network synchrony and overall spike rates. It also affects the kernel predictions via the updated $\bar{G}_{\mathrm{syn} Y X}$ values entering Eqs. (7) and (28).

Our findings, summarized in Figure 11, show in panel B that reduced connection weights result in a marked increase in oscillatory activity in the $75 \mathrm{~Hz}$ range and an overall increase in typical firing rates as given above the spike raster plots in panel A, while the opposite effect is seen when increasing weights. This phenomenom can be explained by decorrelation by inhibitory feedback (Tetzlaff et al. 2012). The increase in oscillatory activity seen with reduced connection weights both reduces temporal agreement $\left(R^{2}\right.$, panel C) and is also associated with reduced signal amplitudes as measured 
using the $r_{\mathrm{STD}}$ metric (panel D). Increasing the maximum conductances in our MC neuron networks has less adverse effect on the accuracy of the kernel-based approximations but this observation may be counteracted also by the overall suppression of oscillations and firing rates. The aggregated $R^{2}$ and $r_{\mathrm{STD}}$ values in panel $\mathrm{E}$ show that all but $J=0.25$ falls within the same range.

As a final test we also recompute the accuracy metrics for $P_{z}(t)$ in Figure 11F. Also here decreasing connection strengths (but increasing synchrony) adversely affects $r_{\mathrm{STD}}$ more so than the $R^{2}$ metric. Increasing connection weights only affects the performance of the kernel prediction method to a minor degree.

For the signals we consider, the kernel based approach works well for the low-frequency signal components, reflected in the slightly improved $R^{2}$ values.
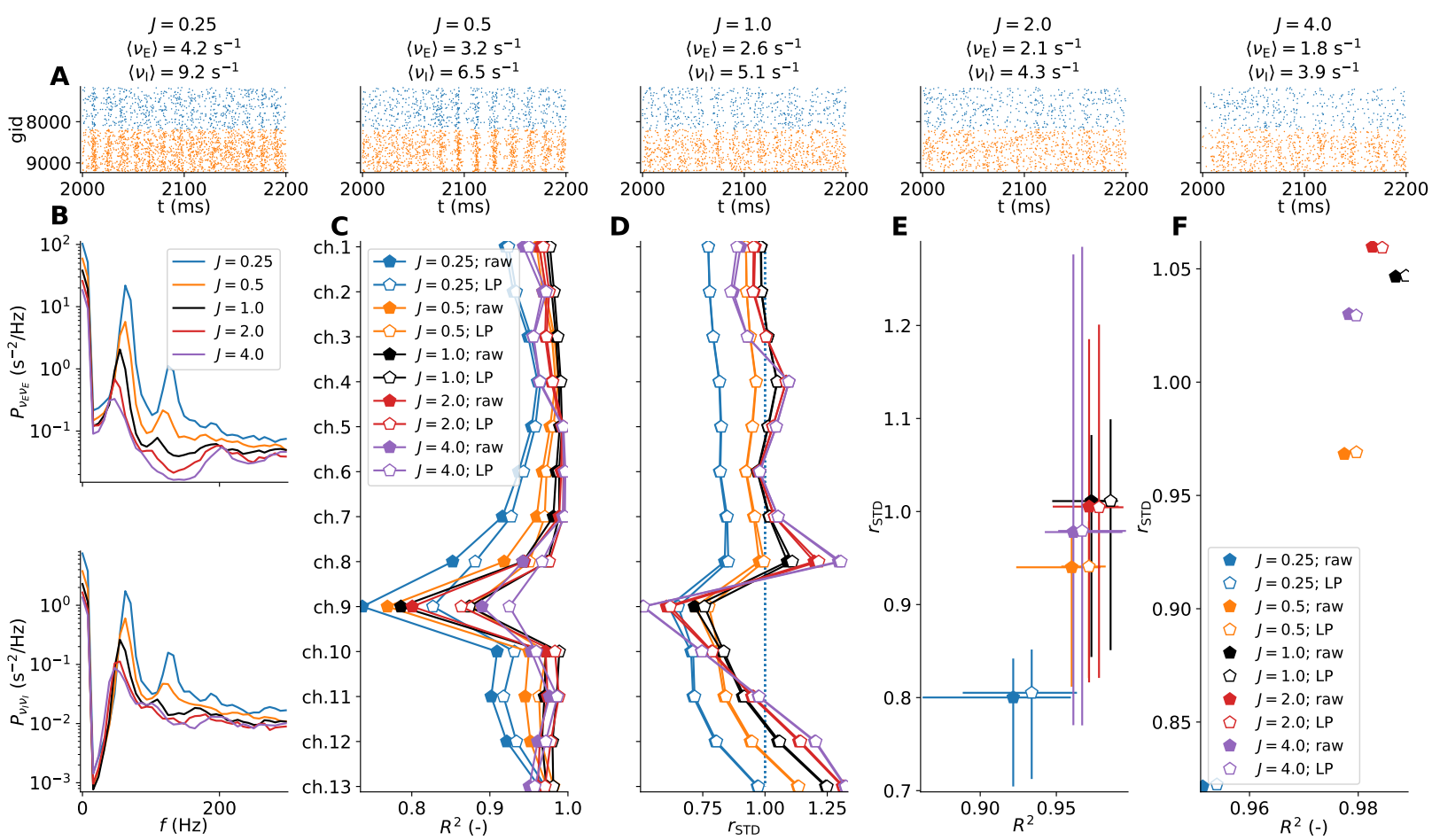

Figure 11: Effect of perturbed MC-network connection weights on kernel-based signal predictions. (A) Mean population spike rates and raster plots $(N=1024$ spike trains in each population). The scaling factor $J$ rescales all connection weights in each network simulation. $J=1$ corresponds to our unperturbed reference network. (B) Effect of uniform scaling of connection weights on firing rate power spectra of populations 'E' (top) and 'I' (bottom). (C) Accuracy of kernel predictions in terms of $R^{2}$ and (D) $r_{\text {STD }}$ for kernel-based predictions of raw- and low-pass filtered extracellular potentials. Here, kernels are in each case computed using 'biophys:frozen' ion channel dynamics accounting for changes in the leak conductance from synaptic conductances ( $g_{-}$eff $:$True). (E) Aggregated $R^{2}$ and $r_{\text {STD }}$ values (median, $10 \%$ and $90 \%$ percentiles) across electrode channels. (F) $r_{\text {STD }}$ vs. $R^{2}$ computed for the $z$-component of the raw- and low-pass filtered current dipole moment $\left(P_{z}(t)\right)$.

\subsection{Methods performance using biophysically detailed cell models}

So far in this paper we kept the neuron and network model descriptions at a deliberately low level of complexity. Biological neurons are commonly modeled at a much greater level of biophysical detail both in terms of geometry and in terms of heterogeneous types of ion channels, and are also used in large scale MC neuron network simulation studies (e.g., Markram et al. 2015). Here, we therefore investigate how well extracellular signals of neural activity can be captured using the linearization steps introduced for networks using stylized neurons, in networks incorporating biophysically detailed neuron models. For this purpose, we replace the excitatory neurons in our network with a rat layer 5b pyramidal cell model by Hay et al. 2011, and repeat the analyses of hybrid- and kernel-based approximations. The network parameterization is kept identical, except for an increased indegree of external input to this excitatory population in order to preserve overall firing rates (see Section 2.1.3 for details). The hybrid and kernel based approximations rely 
on linearized variants of the biophysically detailed neuron model, showcased in Section 3.1. To emphasize on effects explained by this change of model neuron, we exclude signal contributions by transmembrane currents of inhibitory neurons in the analysis.

\subsubsection{Hybrid scheme signal predictions}

First, we consider the hybrid scheme setup, where spike events of the recurrent network is used for synaptic activation times in populations of neurons but without recurrent connections, and repeat the experiments of Section 3.3. Summarized in Figure 12; If the effective leak conductance is not accounted for (g_eff : False, panel A), signal amplitudes are clearly overestimated. Predictions are in better agreement with the ground truth when the leak conductance contribution from synaptic activation is accounted for ( $g$ _eff : True, panel B). In contrast to the previous model setup, the ground truth extracellular potential signals contain prominent extracellular spike contributions, in particular across the soma-proximal ch. 9-12. In terms of choice of linearized membrane dynamics, the visual differences are minuscular resulting in overlapping lines in the plot.

\subsubsection{Kernel based signal predictions}

Next, we compare spike-to-signal impulse response functions ('kernels') computed via the hybrid scheme setup and the computationally fast deterministic method. The resulting set of kernels for connections onto the excitatory population in Figure 13, showcase that the deterministic method yields quantitatively similar results as the corresponding 'hybrid scheme' based method. The differences can in part be explained by the fact that the hybrid implementation employ discrete synapse and cell placements in space, as they occur in the recurrent network used for ground truth signal generation, while the direct method only accounts for the underlying distributions used to set up the recurrent network in the first place. Note that with the reconstructed neuron there is a higher degree of freedom in terms of discrete synapse placements compared to the ball-and-stick neuron. The current dipole moment kernels for the E-to-E and I-to-E projections remain very similar, although visible differences now occur between the quasi-linearized and passive-frozen model neurons.

The corresponding signal predictions using these sets of kernels in combination with population firing rates are shown Figure 14. Again, visual inspection reveal only small differences. Thus, we recompute our accuracy metrics as shown in Figure 15 including also the hybrid scheme predictions in Figure 12 in our analysis. Similar to what is achieved with ball-and-sticks neuron networks, the projected accuracies for all approximations remain clustered together if the effective membrane leak conductance is accounted for. However, the $R^{2}$ metric is reduced, particularly in the uppermost channels, while $r_{\mathrm{STD}}$ is increased irrespective of signal type compared to our earlier results. The different approximations are observed to perform better in the low-frequency range as contributions by presynaptic APs in the ground truth signals are attenuated ('LP' vs. 'raw' data, respectively).

Overall, these observations of slightly reduced performance compared to the ball-and-sticks cases should be of no major surprise, as this biophysically detailed cell model by Hay et al. 2011 has a much more elaborate dendritic structure with many thin sections and many more degrees of freedom in terms of voltage-gated ion channels. Thus, the somatic voltage value we chose for linearized synapse and membrane dynamics may poorly represent voltage fluctuations and deviations which may be present, particularly in dendrites located remotely from the soma. Still, all approximations are able to provide excellent insight into the spatiotemporal properties of the extracellular potential and current dipole moment (and by extension EEG and MEG like signals computed from it), more so in the low-frequency band.

\subsection{Kernel-based signal predictions from point-neuron network model}

So far we have shown that estimates of linear spike-to-signal impulse-response functions ('kernels') allow for accurate approximations to different signals by convolving population firing rates with the appropriate sets of kernels and summing the contributions. So what does this allow for?

One major benefit is that spiking dynamics can with ease be modeled using recurrently connected networks employing simplified neuron representations, like leaky integrate-and-fire (LIF) point neurons and variants thereof. Recurrent network models using biophysically detailed MC neuron models (e.g., Markram et al. 2015; Billeh et al. 2020) are, in contrast, intrinsically more difficult to develop due to their vast number of parameters (Almog and Korngreen 2016), are comparably slow to simulate even on large scale HPC facilities, less amenable for analytical analysis, and henceforth difficult to constrain into reasonable network states resembling experimental data. Point-neuron networks mediates all of these important issues. In addition, systematic reductionist approaches applied to MC neuron networks allow for capturing their spiking dynamics in equivalent few-compartment or point-neuron networks (Wybo et al. 2015; Rössert et al. 2017; Amsalem et al. 2020). But point-neuron networks do not allow for computing the distribution of transmembrane currents in space needed for signal predictions, as all in- and out-going currents sum to zero in a point 


\section{A}
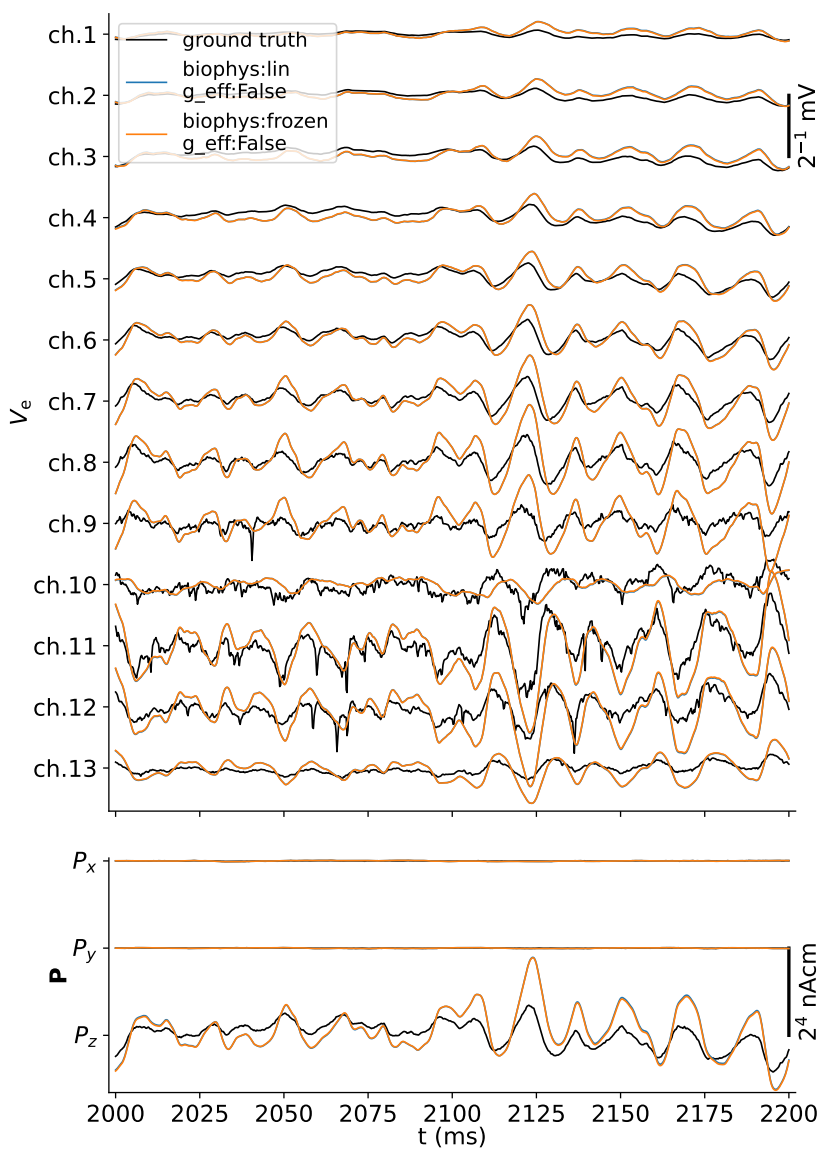

B
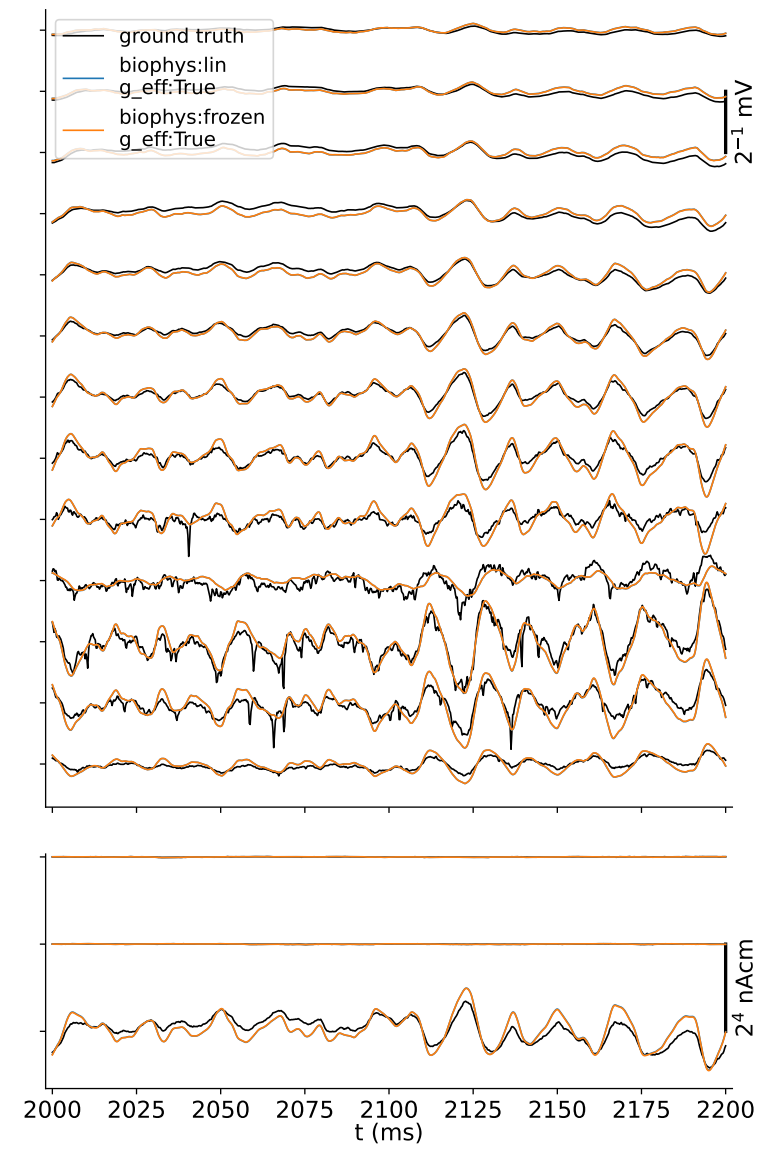

Figure 12: Ground truth signals vs. hybrid scheme approximations. Same as Figure 4, but with the excitatory cell model being replaced by a biophysically detailed pyramidal cell model (Hay et al. 2011), and accounting only for contributions by transmembrane currents of this excitatory population.

(Hagen et al. 2016). Using our direct and deterministic method we can, however, predict sets of kernels $\hat{H}_{Y X}(\mathbf{R}, \tau)$ for each connectivity pathway via a single $\mathrm{MC}$ neuron simulation in order to predict extracellular signals from simplified networks.

As a proof of principle of this methodology, we constructed a point-neuron network of the same size as our reference MC-neuron networks and fit its parameters in order to mimic our reference network's averaged firing rates and rate power spectra (see Section 2.1.4 for details). Showcased in Figure 16, the point-neuron network state is asynchronous and irregular (AI) with some oscillations present in the corresponding spike count histogram (panel A), similar to our reference network. We here also showcase the different signal contributions by each pathway (E-to-E and so forth) in panels B-E, using the set of kernels displayed in Figure 7 and discussed in Section 3.4.2. The summed contributions are shown in panel F. Here, there are no ground truth signals to compare with directly, but the extracellular potential signal vary across time and space in an expected manner, and closely resembles the signals obtained by the I-to-E pathway. Signal amplitudes are also in the expected ranges set by our MC-neuron network simulations.

As a final remark, we here compute the firing rates and signals 'live' while the network simulation is running. To reiterate, the kernels are always causal, that is, equal to zero for any time less than the minimum conduction delay in the network and in practice of finite duration. This causal relationship allows for treating the sets of kernels as finite-impulse-response (FIR) filter coefficients, which are here applied via a custom network node that receives incoming spike events from each population while the simulation is running and outputs continuous signals representing the temporally filtered spike events. For this purpose a proof of principle FIR filter network node is implemented for the NEST simulator (Gewaltig and Diesmann 2007; Deepu et al. 2021) via the NESTML description language (Plotnikov et al. 2016; Nagendra Babu et al. 2021) as detailed in Section 2.6. 

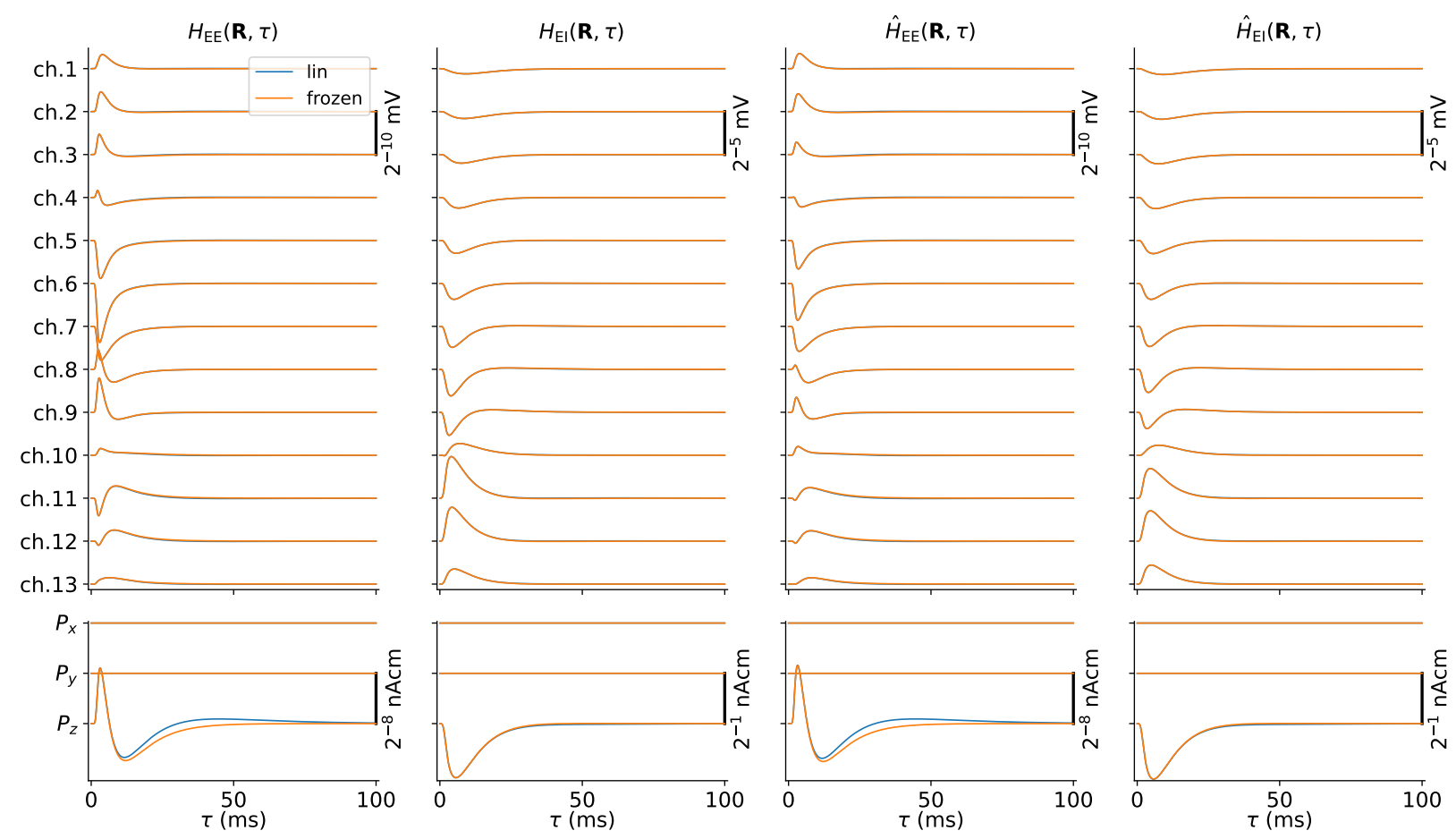

Figure 13: Spike-LFP impulse response function averages and predictions. Similar to panels in Figure 5 and Figure 7, but but with the excitatory cell model being replaced by a biophysically detailed pyramidal cell model (Hay et al. 2011).

\section{Discussion}

\section{Summary of findings}

The main results presented throughout this paper can be summarized as follows: First, an assessment on the validity of different prediction schemes for extracellular signals assuming linearity between times of presynaptic action potentials ('spikes') and corresponding extracellular signals that occur due to evoked transmembrane currents on the postsynaptic neuronal populations. Our finding is that the linearity assumption is valid, if all contributions from linearized membrane and synapse conductances are accounted for, resulting in accurate signal predictions.

Secondly, identification of the critical role of the effective membrane time constant due to persistent activation of recurrent and external synapses on predicted signals. We found that simply approximating conductance-based synapses by current-based synapses without accounting for the time-averaged synaptic conductances resulted in overestimated amplitudes and poorer temporal accuracy of the approximated signals.

Third, a new, fast and accurate method to compute extracellular signals from biologically inspired finite-sized neuronal network models. This new method proposal allows for computing averaged spatiotemporal spike-to-signal impulse response functions ('kernels') for connections between pre- and postsynaptic populations, by accounting for distributions of cells and synapses in space, linearized synapse and membrane dynamics, overall connection probabilities, distributions of synapses per instantiated connection and connection delay distributions. As the sets of computed kernels are causal and linearly map population spike events to the corresponding signals, it allows for signal predictions as in a linear time-invariant (LTI) causal system, that is, by treating the sets of spatiotemporal kernels as finite impulse response (FIR) filter coefficients applied to corresponding firing rates of presynaptic populations. The kernel-based predictions are as accurate as a hybrid scheme explicitly accounting neuron and synapse placements in space (Hagen et al. 2016), but significantly faster.

We developed and evaluated the methodology based on recurrently connected reference networks of MC neurons. For simplicity, we initially opted for phenomenological ball-and-sticks MC neuron models with active voltage-gated ion channels distributed all over, representing each population of excitatory (E) and inhibitory (I) neurons. Synapses are conductance-based. We show that the proposed methodology is feasible with perturbed network states, as well as for 


\section{A}
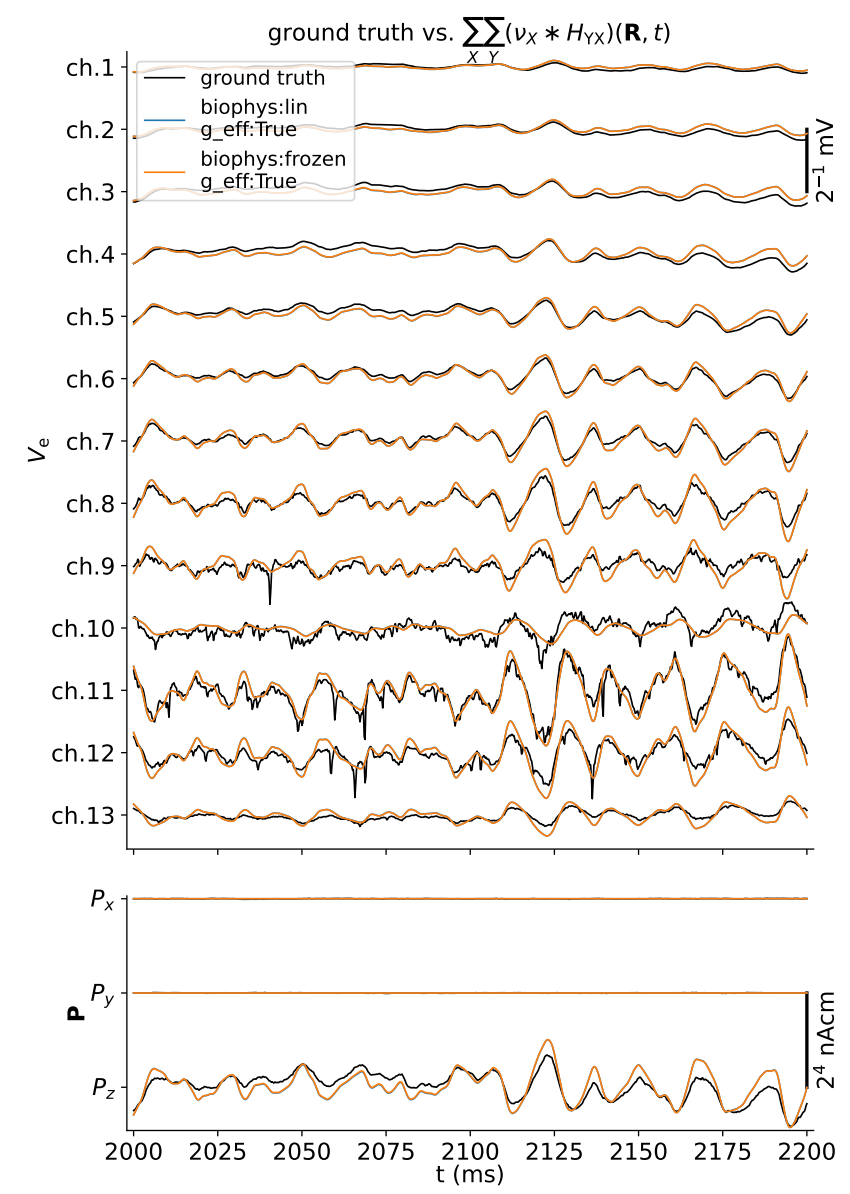

B
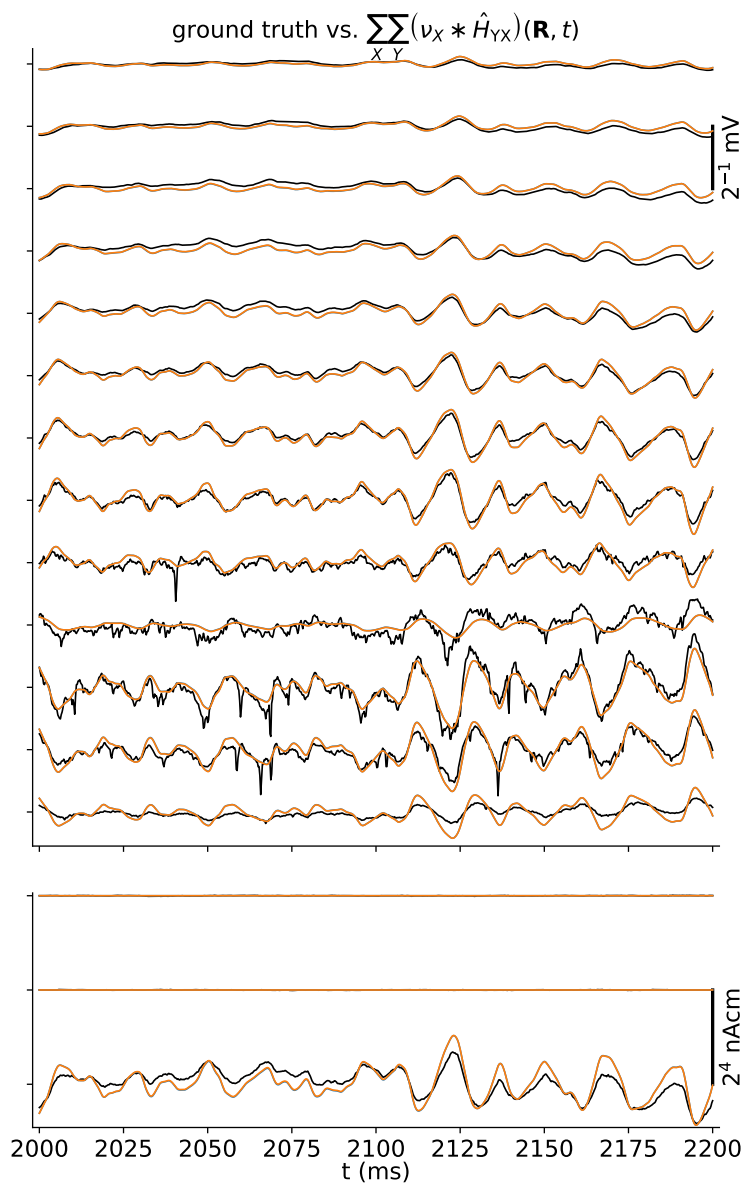

Figure 14: Ground truth signals vs. kernel-based approximations. Same as Figure 8, but with the excitatory cell model being replaced by a biophysically detailed pyramidal cell model (Hay et al. 2011). Here, only signal contributions by transmembrane currents of the updated excitatory population are accounted for.

cases where the the 'E' cell population is replaced by a biophysically detailed neurons (Hay et al. 2011) at a level of description similar to neuron models implemented in high-profile biophysically detailed network modeling efforts (e.g., Markram et al. 2015; Billeh et al. 2020).

As a final proof of principle for the kernel-prediction methodology, we apply a suitable set of kernels for forward-model predictions from spiking activity in a spiking point-neuron network model. For this network model, the kernels are applied via a FIR filter network node receiving presynaptic spike events applying the filter coefficients for continuous signal predictions during the course of the simulation. The resulting signals resemble corresponding ground truth signals of the reference MC neuron network.

\section{Kernels versus other estimation methods}

The sets of spike-signal kernels we compute using our proposed methodology should not be confused with corresponding spike-triggered averaged signals (e.g., Nauhaus et al. 2009; Denker et al. 2011), which are intrinsically affected by ongoing network activity, that is, spike train correlations, as previously shown in Hagen et al. 2016. Even if both are linear measures, the spike-triggered averaged signal will most likely be non-causal and depend on network state, although one exception may be if the spiking activity of the trigger neuron is approximately uncorrelated with the ongoing activity. The latter scenario may occur for instance for spontaneous activations of neurons in one brain region (e.g., thalamus) projecting to another area (e.g,. somatosensory or visual cortex, see Swadlow et al. 2002; Stoelzel et al. 2008; Bereshpolova et al. 2019). This so-called monosynaptic, also referred to as unitary (e.g., by Bazelot et al. 2010) extracellular response is recently modeled in detail (Hagen et al. 2017), then using conductance based synapses but 

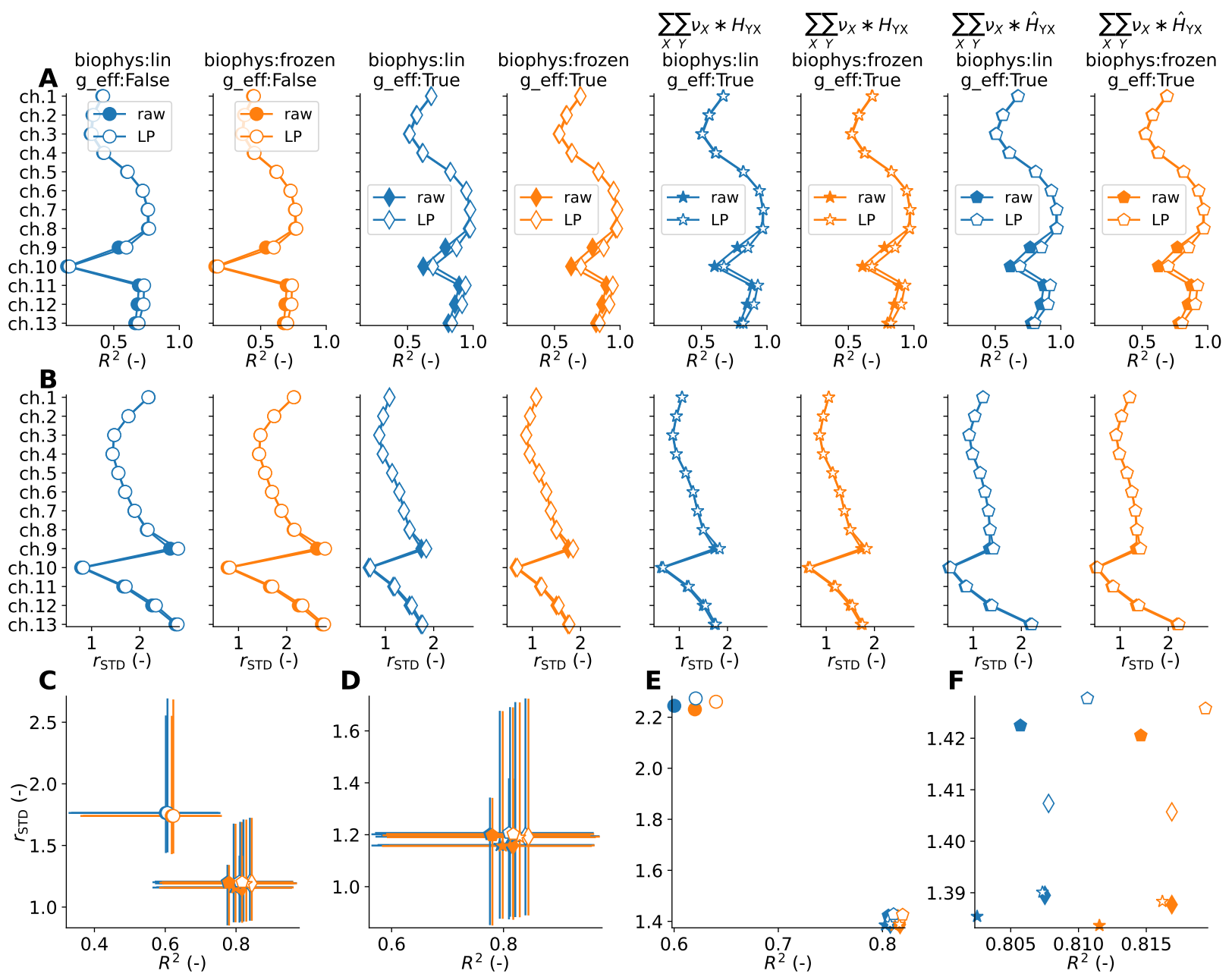

Figure 15: Accuracy of signal predictions vs. ground truth. Same as Figure 9, but with the excitatory cell model being replaced by a biophysically detailed pyramidal cell model (Hay et al. 2011), and accounting only for contributions by transmembrane currents of this updated excitatory population.

with passive membrane time constants fitted to available experimental and published data. A similar effort to compute such responses in hippocampus was recently published by Teleńczuk et al. 2020b. Teleńczuk et al. 2020a proposed using such responses then fitted to spatiotemporal kernel shape functions for excitatory and inhibitory presynaptic units in order to compute LFP signals in point-neuron network models. The sets of kernels we compute do not assume a particular shape, but are derived from biophysics of the network, and can be recomputed for other networks and populations.

Other, even simpler estimation methods for extracellular potential time series was proposed by Mazzoni et al. 2015, recently extended to EEG signals by Martínez-Cañada et al. 2021, by approximating signals by weighted and timeshifted sums of excitatory and inhibitory synaptic currents measured in the network simulation. In contrast to the hybridand kernel-based approaches considered here, these simplified approximations do not explicitly account for any effects on the predicted signals from the neuronal morphologies, ion-specific channels and the VC forward model. They also do not account for any signal variation in space except if combined with some position-dependent scaling factor, and the physical units of the predicted signals can be considered arbitrary. These simplified schemes may still be considered a major improvement over ad hoc approaches equating firing rates or averaged somatic potentials to extracellular signals (Mazzoni et al. 2015; Martínez-Cañada et al. 2021). In case of scalp EEG and MEG signal predictions mainly the current dipole moment components normal to the cortical tissue surface may be predicted with reasonable accuracy and be combined with an appropriate head forward model (Næss et al. 2021), allowing for respective signal predictions along the scalp's surface. 


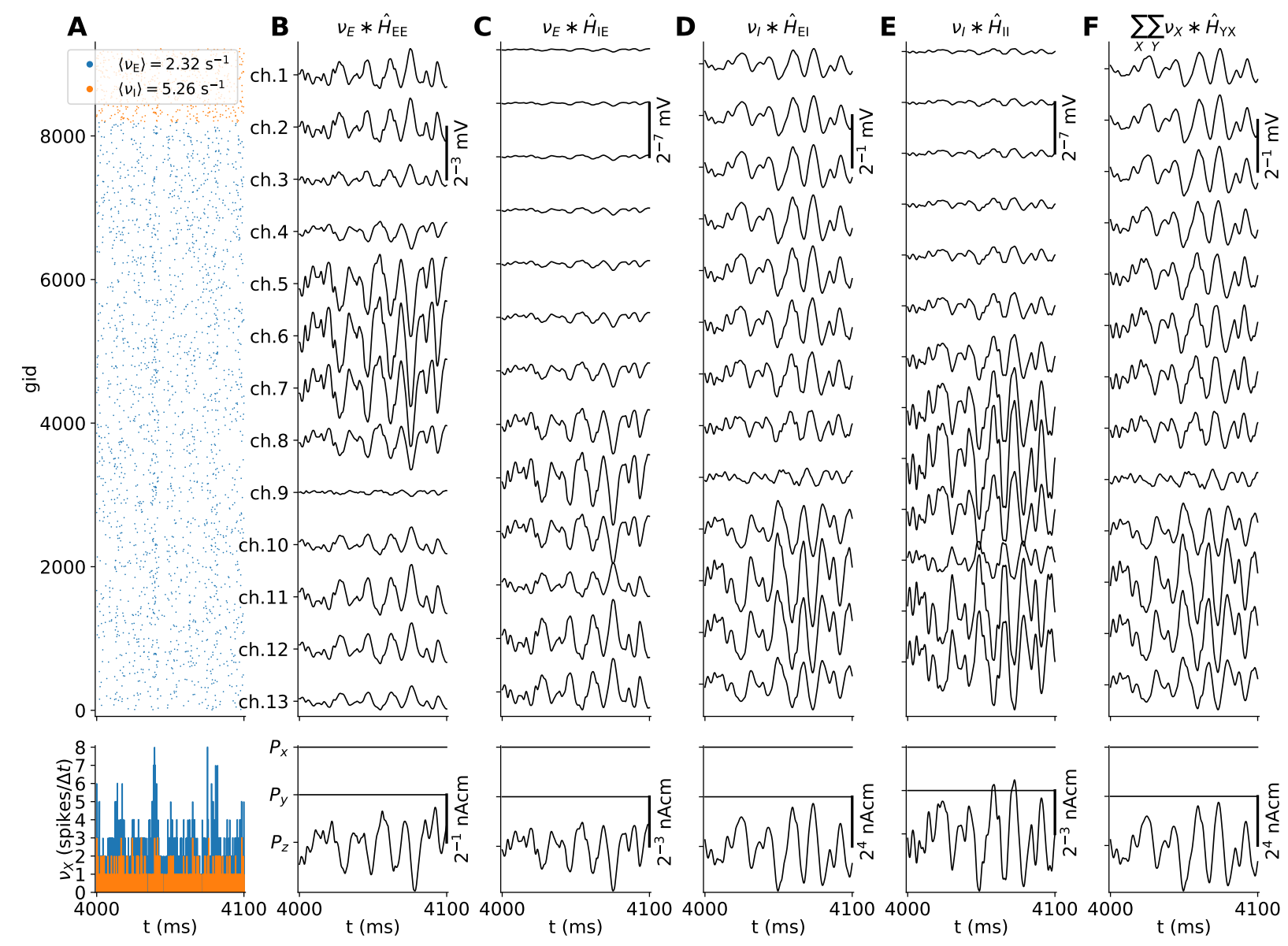

Figure 16: LIF network spiking activity and forward-model predictions. (A) Spiking activity and average spike rates of the excitatory (E) and inhibitory (I) populations of a point neuron network simulation (top), with spike counts in bins of width $\Delta t$ (bottom). (B-E) Contributions to the extracellular potential (top) and current dipole moment (bottom) by the $\mathrm{E}$ to $\mathrm{E}$ connection, $\mathrm{E}$ to I connection, I to E connection and I to I connection, respectively. The signals are equivalently computed as the convolution between the presynaptic population spike count histogram and corresponding signal kernel approximations using a FIR filter implementation concurrently with the spiking simulation. The kernels used are shown in Figure 7. (F) Sum over all contributions in panels B-E.

\section{Extensions and future works}

One main novelty reported here is the proposed method for directly computing kernels that facilitate efficient calculations of extracellular signals from population spike rates, as well as a reference implementation in the Python package LFPykernels. While our present application of this framework is to quite simplified two-population recurrent networks of MC neuron models, the present implementation is applicable to networks with many more populations. One could for instance mimic the laminar topology of cortical microcircuits, where each layer consists of different populations representing the heterogeneous types of cells within each layer as in Markram et al. 2015and Billeh et al. 2020. Based on available anatomical and electrophysiological constraints either from experiments or detailed models themselves, signal kernels of interest can then be computed for the different connections independently of simulations of recurrent network spiking activity. The latter step may then even use simplified neurons (e.g., spiking point neurons) thus negating the need for HPC facilities. Extracellular signal predictions can be incorporated in the running simulation as we have demonstrated here (see Section 3.9), or post simulation by computing population spike rates from recorded spike events, filter these and summing up all contributions.

While we have here mainly focused on the methodology and less on overall simulation speeds, we note that potential speedup can be of several orders of magnitude. The typical simulation times for the recurrent network with biophysically detailed pyramidal neuron models (see Section 3.8) we observe are around 4400 s multiplied by 1024 physical cores for 
$12 \mathrm{~s}$ of biological time on the HPC resource, while the corresponding set of kernel predictions take around $150 \mathrm{~s}$ on a laptop computer using a single physical core (see Section 2.7 for details). This number can potentially be reduced substantially if the numerical integration of Equation (35) on a per compartment basis can be replaced by a closed form (the MC neuron simulations of transmembrane currents are quite brief). Further reductions in prediction times may involve other trivial parallelization schemes, as kernels for different connections can be computed fully independent of each other, which is also the case for different spatial components of each spatiotemporal kernel. Simulation times and resources required for spike times in equivalent networks of the same size using simplified neurons (i.e., few-compartment and point-neuron models) are also substantially less compared to the biophysically detailed case. For the point-neuron network incorporating the FIR filter operations used here, the respective network build and simulation times were around $8 \mathrm{~s}$ and $235 \mathrm{~s}$ with single-threaded execution on a laptop. Thus the serial time to solution is reduced by a factor $\sim 10^{4}$ compared to the MC network simulation. Hence, the avenue of biophysics-based forward model predictions of extracellular signals in present and future large-scale networks with millions of spiking point neurons and beyond (e.g., Schmidt et al. 2018) is opened.

Still, there are multiple scenarios where our proposed methodology could use either further development or validation. Presently we investigate the method for networks with a columnar (cylindrical) organization and no distance-dependency for connections in terms of connection probabilities, synaptic conductances and axonal transmission delays within the column like geometry. Large scale recurrent network models with (lateral) distance-dependent connectivity and periodic boundary conditions spanning multiple $\mathrm{mm}$ of cortical area has been proposed at various levels of description (e.g., Senk et al. 2018b; Billeh et al. 2020), but so far our proposed kernel prediction method is neither developed for nor validated against such models. So far, such lateral distance-dependent connectivity was only accounted for in a phenomenological kernel based prediction model (Senk et al. 2018a), or for an experimentally derived kernel-based method (Teleńczuk et al. 2020a).

Furthermore, we assume recurrent networks with static connection weights. But synapses may be subject to various weight dynamics such as short term plasticity (STP) with activity-dependent facilitation and depression, stochasticity, spike-timing-dependent plasticity (STDP) (see e.g., Magee and Grienberger 2020), as well as structural plasticity (Lamprecht and LeDoux 2004). Out of these, stochasticity is perhaps easier dealt with if probabilities of synaptic activations are known and independent of activation rate by scaling the corresponding kernel amplitudes accordingly. Weight changes due to STP is governed by presynaptic activation intervals hence the average connection weights for kernel predictions can be determined for known averaged presynaptic rates. STDP may be harder to account for, but due to the much longer time scales the option to monitor connection weights during the course of simulation could allow for recomputing kernels and apply them on each simulation segment.

Our analyses also demonstrate that the accuracies of kernel-based signal predictions versus corresponding reference signals can be expected to drop when the degree of synchrony and/or firing rates in the network increases (see Section 3.7), which we achieved by rescaling all synaptic connection weights by the same factor. Our reference network generates oscillations in the gamma range $(\sim 75 \mathrm{~Hz})$ when driven by external fixed-rate Poisson processes, but we expect to observe similar effects on prediction errors for networks with non-stationary activity. Such non-stationarities may include up-and-down states (Wilson 2008) or result from variable-rate external drive (e.g., representing sensory input), as the choice for membrane potential when linearizing synapse and ion-channel dynamics may indeed affect the kernel predictions. Still, our hope is that the methodology can still give excellent qualitative insight in extracellular signals from networks expressing non-stationary behavior.

Contrary to our starting point, a recurrent MC neuron network, forward model predictions from recurrent point-neuron networks poses a potential challenge due to their inherent lack of detail. Their descriptions may contain no spatial information even if the network is supposed to mimic a particular brain area, such as the generic somatosensory cortex column model proposed by Potjans and Diesmann 2014, representing the local circuitry under a $1 \mathrm{~mm}^{2}$ patch of cortical surface. To compute extracellular potentials from this model spatial information in terms of neuron geometries and depth-dependencies for synaptic placements must be determined based on available anatomical data (see Hagen et al. 2016 for details). Similarly, the present kernel predictions requires multicompartment neuron models representative of each population, and statistical distributions describing placements of cells within each population in space, placements of synapses across the neuron models for each pre and postsynaptic population, numbers of synapses per instantiated connection. Other parameters may (or may not) be derived from the point-neuron network description, such as conduction delay distributions and synaptic parameters. Some may be derived from its activity, such as population firing rates. As such, multiple concurrent efforts aim to amass such anatomical and electrophysiological detail for different brain regions and species with corresponding tools for enquiring the data (see e.g., Grillner et al. 2016; Koch and Jones 2016; Amunts et al. 2016; Bjerke et al. 2018; Gouwens et al. 2019). Such data may be used to derive suitable kernels.

For rate-based frameworks aiming to explain activity in terms of population firing rates in finite-sized populations (see e.g., Gerstner 2014; Cain et al. 2016; Dumont et al. 2017; Schwalger et al. 2017), special attention should also be taken. 
Unless the rate-based models are derived using bottoms-up approaches in contrast to heuristics, use of our proposed kernel prediction scheme also necessitates specifying parameters such as population cell counts and pairwise connection probabilities. Otherwise, the resulting kernel amplitudes can be considered arbitrary. If such parameters indeed can be determined, we do not see any principled reasons why one could not apply the kernels with continuous population rate predictions as we already demonstrated with temporally binned population spike rates computed from spiking networks.

Further extensions of our kernel estimates for continuous neural fields equations aiming to explain activity across space (Ermentrout and Terman 2010) should be based on and validated via aforementioned laminar network models incorporating lateral distance-dependent connectivity routines. For discretized spiking point-neuron network models with distance-dependent connectivity, Senk et al. 2020 derived corresponding neural field equations. Developments in this direction is required for simulation frameworks such as 'The Virtual Brain' (TVB, Leon et al. 2013) aiming to relate firing rates across brain areas also with extracellularly recorded signals such as the EEG, as well as similar tools aimed towards clinical use (Jirsa et al. 2017).

Finally, we have considered only postsynaptic contributions from synaptic activations to signals predicted using the hybrid scheme or kernel-based methods. These approaches are therefore better able to capture the low-frequency parts of the signals as most clearly demonstrated in our simulations using the biophysically detailed layer 5 pyramidal neuron which resulted in clearly visible extracellular spikes. One could potentially account for signal contributions by presynaptic events such as somatic APs, backpropagating APs, $\mathrm{Ca}^{2+}$ and NMDA spikes by computing and superposing the extracellular signatures of each event to the signals considered here, in case the network model accounts for times of such events. Taking such steps would result in non-causal kernel contributions and would require additional validation against network models using biophysically detailed neuron models expressing such phenomena. It should however be feasible to incorporate, and could improve the accuracy of the present implementation around frequencies where spike contributions are assumed to dominate in the extracellular signals.

\section{Conclusion}

Many of the research successes in the physical sciences have come from an interplay between modeling and experiments where predictions between physics-based candidate models have been systematically compared with experiments in an iterative back-and-forth loop. This approach is sometimes referred to as the 'virtuous loop' or circle (Hassabis et al. 2017). For large-scale network models in the brain this approach has until now been hampered by the lack of physics-based forward models able to predict mesoscopic and macroscopic brain signals like LFPs and EEGs (Einevoll et al. 2019). We believe that the kernel-based approach presented here could be an important step forward for making such model-based predictions feasible, thus paving the way for use of the virtuous loop also in large-scale network neuroscience.

\section{Acknowledgements}

This work was supported by the European Union Horizon 2020 Research and Innovation Programme under Grant Agreement No. 785907 and No. 945539 Human Brain Project (HBP) SGA2 and SGA3 [EH,TVN,GH,PNB,CL,AM,GTE]. We also acknowledge the use of Fenix Infrastructure resources, which are partially funded from the European Union's Horizon 2020 Research and Innovation Programme through the ICEI Project under the Grant Agreement No. 800858 [EH,SHM,TVN,GH,GTE]; The Helmholtz Alliance through the Initiative and Networking Fund of the Helmholtz Association and the Helmholtz Portfolio theme Supercomputing and Modeling for the Human Brain [PNB,CL,AM]; and The Excellence Strategy of the Federal Government and the Länder [G:(DE-82)EXS-PF-JARA-SDS005, G:(DE82)EXS-SF-neuroIC002] [PNB,CL,AM].

\section{Authors contributions}

EH, SMH, TVN, GH, GTE conceived and conceptualized the project. EH drafted the manuscript. SMH, TVN, GH, PNB, CL, AM, GTE cowrote the manuscript. PNB and CL adapted and implemented the FIR filter implementation in NESTML. EH wrote and ran all remaining simulation, analysis and plotting codes for this paper.

\section{References}

Ahmadi, Nur, Timothy G. Constandinou, and Christos-Savvas Bouganis (2021). "Inferring entire spiking activity from local field potentials". In: Scientific Reports 11.1. DOI: 10.1038/s41598-021-98021-9. 
Akar, Nora Abi, Ben Cumming, Vasileios Karakasis, Anne Kusters, Wouter Klijn, Alexander Peyser, and Stuart Yates (2019). "Arbor - A Morphologically-Detailed Neural Network Simulation Library for Contemporary HighPerformance Computing Architectures". In: 2019 27th Euromicro International Conference on Parallel, Distributed and Network-Based Processing (PDP). IEEE. DOI: 10.1109/empdp. 2019.8671560.

Almog, Mara and Alon Korngreen (2016). "Is realistic neuronal modeling realistic?" In: Journal of Neurophysiology 116.5, pp. 2180-2209. DOI: 10.1152/jn.00360.2016.

Amsalem, Oren, Guy Eyal, Noa Rogozinski, Michael Gevaert, Pramod Kumbhar, Felix Schürmann, and Idan Segev (2020). "An efficient analytical reduction of detailed nonlinear neuron models". In: Nature Communications 11.1. DOI: $10.1038 / \mathrm{s} 41467-019-13932-6$.

Amunts, Katrin, Christoph Ebell, Jeff Muller, Martin Telefont, Alois Knoll, and Thomas Lippert (2016). "The Human Brain Project: Creating a European Research Infrastructure to Decode the Human Brain”. In: Neuron 92.3, pp. 574581. DOI: $10.1016 / j$.neuron. 2016.10.046.

Bazelot, Michaël, Céline Dinocourt, Ivan Cohen, and Richard Miles (2010). "Unitary inhibitory field potentials in the CA3 region of rat hippocampus". In: Journal of Physiology 588.12, pp. 2077-2090. ISSN: 00223751. DOI: 10.1113/jphysiol.2009.185918.

Bereshpolova, Yulia, Carl R. Stoelzel, Chuyi Su, Jose Manuel Alonso, and Harvey A. Swadlow (2019). "Activation of a Visual Cortical Column by a Directionally Selective Thalamocortical Neuron". In: Cell Reports 27.13, 3733-3740.e3. ISSN: 22111247. DOI: 10.1016/j . celrep.2019.05.094.

Berger, Hans (1929). "Über das Elektrenkephalogramm des Menschen". In: Archiv für Psychiatrie und Nervenkrankheiten 87.1, pp. 527-570. DOI: 10.1007/bf01797193.

Billeh, Yazan N., Binghuang Cai, Sergey L. Gratiy, Kael Dai, Ramakrishnan Iyer, Nathan W. Gouwens, Reza AbbasiAsl, Xiaoxuan Jia, Joshua H. Siegle, Shawn R. Olsen, et al. (May 2020). "Systematic Integration of Structural and Functional Data into Multi-scale Models of Mouse Primary Visual Cortex". In: Neuron 106.3, 388-403.e18. DOI: $10.1016 / j$.neuron. 2020.01.040.

Bjerke, Ingvild E., Martin Øvsthus, Eszter A. Papp, Sharon C. Yates, Ludovico Silvestri, Julien Fiorilli, Cyriel M.A. Pennartz, Francesco S. Pavone, Maja A. Puchades, Trygve B. Leergaard, and Jan G. Bjaalie (2018). "Data integration through brain atlasing: Human Brain Project tools and strategies". In: European Psychiatry 50, pp. 70-76. DOI: $10.1016 / j$. eurpsy. 2018.02.004.

Blank, Julian and Kalyanmoy Deb (2020). "Pymoo: Multi-Objective Optimization in Python". In: IEEE Access 8, pp. 89497-89509. DOI: 10.1109/access . 2020.2990567.

Brunel, Nicolas (2000). "Dynamics of Sparsely Connected Networks of Excitatory and Inhibitory Spiking Neurons". In: Journal of Computational Neuroscience 8.3, pp. 183-208. DOI: 10.1023/a:1008925309027.

Cain, Nicholas, Ramakrishnan Iyer, Christof Koch, and Stefan Mihalas (2016). "The Computational Properties of a Simplified Cortical Column Model”. In: PLOS Computational Biology 12.9. Ed. by Olaf Sporns, e1005045. DOI: 10.1371/journal.pcbi.1005045.

Carnevale, Nicholas T. and Michael L. Hines (2006). The Neuron Book. Cambridge University Press. 480 pp. ISBN: 9780521843218.

Dai, Kael, Sergey L. Gratiy, Yazan N. Billeh, Richard Xu, Binghuang Cai, Nicholas Cain, Atle E. Rimehaug, Alexander J. Stasik, Gaute T. Einevoll, Stefan Mihalas, et al. (2020). "Brain Modeling ToolKit: An open source software suite for multiscale modeling of brain circuits". In: PLOS Computational Biology 16.11. Ed. by Daniele Marinazzo, e1008386. DOI: $10.1371 /$ journal.pcbi.1008386.

Deb, K., A. Pratap, S. Agarwal, and T. Meyarivan (2002). "A fast and elitist multiobjective genetic algorithm: NSGA-II". In: IEEE Transactions on Evolutionary Computation 6.2, pp. 182-197. DOI: 10.1109/4235.996017.

Deb, Kalyanmoy (June 26, 2001). Multi-Objective Optimization. John Wiley \& Sons. 536 pp. ISBN: 047187339X. URL: https://www.ebook.de/de/product/5833814/deb_multi_objective_optimization.html.

Deepu, Rajalekshmi, Sebastian Spreizer, Guido Trensch, Dennis Terhorst, Stine Brekke Vennemo, Jessica Mitchell, Charl Linssen, Håkon Mørk, Abigail Morrison, Jochen Martin Eppler, et al. (2021). NEST 3.1. DOI: 10 . 5281 / zenodo. 5508805.

Denker, Michael, Sébastien Roux, Henrik Lindén, Markus Diesmann, Alexa Riehle, and Sonja Grün (2011). "The Local Field Potential Reflects Surplus Spike Synchrony”. In: Cerebral Cortex 21.12, pp. 2681-2695. DoI: 10.1093/ cercor/bhr040.

Dumont, Grégory, Alexandre Payeur, and André Longtin (2017). "A stochastic-field description of finite-size spiking neural networks”. In: PLOS Computational Biology 13.8. Ed. by Bard Ermentrout, e1005691. DOI: 10 . $1371 /$ journal.pcbi.1005691.

Dura-Bernal, Salvador, Benjamin A Suter, Padraig Gleeson, Matteo Cantarelli, Adrian Quintana, Facundo Rodriguez, David J Kedziora, George L Chadderdon, Cliff C Kerr, Samuel A Neymotin, et al. (2019). "NetPyNE, a tool for data-driven multiscale modeling of brain circuits". In: eLife 8. DOI: 10.7554/elife. 44494. 
Ecker, Alexander S., Philipp Berens, Georgios A. Keliris, Matthias Bethge, Nikos K. Logothetis, and Andreas S. Tolias (2010). "Decorrelated Neuronal Firing in Cortical Microcircuits". In: Science 327.5965, pp. 584-587. DOI: 10.1126/science.1179867.

Einevoll, Gaute T., Alain Destexhe, Markus Diesmann, Sonja Grün, Viktor Jirsa, Marc de Kamps, Michele Migliore, Torbjørn V. Ness, Hans E. Plesser, and Felix Schürmann (2019). "The Scientific Case for Brain Simulations". In: Neuron 102.4, pp. 735-744. DOI: 10.1016/j.neuron.2019.03.027.

Einevoll, Gaute T., Christoph Kayser, Nikos K. Logothetis, and Stefano Panzeri (2013). "Modelling and analysis of local field potentials for studying the function of cortical circuits". In: Nature Reviews Neuroscience 14.11, pp. 770-785. DOI: $10.1038 / \mathrm{nrn} 3599$.

Einevoll, Gaute T., Klas H. Pettersen, Anna Devor, Istvan Ulbert, Eric Halgren, and Anders M. Dale (2007). "Laminar Population Analysis: Estimating Firing Rates and Evoked Synaptic Activity From Multielectrode Recordings in Rat Barrel Cortex”. In: Journal of Neurophysiology 97.3, pp. 2174-2190. DOI: 10.1152/jn. 00845. 2006.

Ermentrout, G. Bard and David H. Terman (July 1, 2010). Mathematical Foundations of Neuroscience. Springer-Verlag GmbH. 422 pp. ISBN: 9780387877082. URL: https : / / www . ebook. de/de/product/22858535/g_bard_ ermentrout_david_h_terman_mathematical_foundations_of_neuroscience.html.

Gerstner, Wulfram (July 2014). Neuronal dynamics : from single neurons to networks and models of cognition. Cambridge, United Kingdom: Cambridge University Press. 578 pp. ISBN: 1107635195.

Gewaltig, Marc-Oliver and Markus Diesmann (2007). "NEST (NEural Simulation Tool)”. In: Scholarpedia 2.4, p. 1430. DOI: $10.4249 /$ scholarpedia. 1430.

Głąbska, Helena T., Eivind Norheim, Anna Devor, Anders M. Dale, Gaute T. Einevoll, and Daniel K. Wójcik (2016). "Generalized Laminar Population Analysis (gLPA) for Interpretation of Multielectrode Data from Cortex". In: Frontiers in Neuroinformatics 10. DOI: 10.3389/fninf .2016.00001.

Goodman, Steven N., Daniele Fanelli, and John P. A. Ioannidis (2016). "What does research reproducibility mean?" In: Science Translational Medicine 8.341, pp. 12-12. DOI: 10.1126/scitranslmed .aaf5027.

Gouwens, Nathan W., Staci A. Sorensen, Jim Berg, Changkyu Lee, Tim Jarsky, Jonathan Ting, Susan M. Sunkin, David Feng, Costas A. Anastassiou, Eliza Barkan, et al. (2019). "Classification of electrophysiological and morphological neuron types in the mouse visual cortex". In: Nature Neuroscience 22.7, pp. 1182-1195. DOI: 10.1038/s41593019-0417-0.

Grillner, Sten, Nancy Ip, Christof Koch, Walter Koroshetz, Hideyuki Okano, Miri Polachek, Mu ming Poo, and Terrence J Sejnowski (2016). "Worldwide initiatives to advance brain research". In: Nature Neuroscience 19.9, pp. 1118-1122. DOI: $10.1038 / \mathrm{nn} .4371$.

Hagen, Espen (2021). LFPykernels. DOI: 10.5281/ZENODO. 5720619. URL: https://doi.org/10.5281/ZENODO. 5720619.

Hagen, Espen, David Dahmen, Maria L. Stavrinou, Henrik Lindén, Tom Tetzlaff, Sacha J. van Albada, Sonja Grün, Markus Diesmann, and Gaute T. Einevoll (2016). "Hybrid Scheme for Modeling Local Field Potentials from Point-Neuron Networks". In: Cerebral Cortex 26.12, pp. 4461-4496. DOI: 10.1093/cercor/bhw237.

Hagen, Espen, Janne C. Fossum, Klas H. Pettersen, Jose-Manuel Alonso, Harvey A. Swadlow, and Gaute T. Einevoll (2017). "Focal Local Field Potential Signature of the Single-Axon Monosynaptic Thalamocortical Connection". In: Journal of Neuroscience 37.20, pp. 5123-5143. DOI: 10.1523/jneurosci.2715-16.2017.

Hagen, Espen, Solveig Næss, Torbjørn V. Ness, and Gaute T. Einevoll (2018). "Multimodal Modeling of Neural Network Activity: Computing LFP, ECoG, EEG, and MEG Signals With LFPy 2.0". In: Frontiers in Neuroinformatics 12, p. 92. DOI: 10.3389/fninf.2018.00092.

Hagen, Espen, Torbjørn V. Ness, Amir Khosrowshahi, Christina Sørensen, Marianne Fyhn, Torkel Hafting, Felix Franke, and Gaute T. Einevoll (2015). "ViSAPy: A Python tool for biophysics-based generation of virtual spiking activity for evaluation of spike-sorting algorithms". In: Journal of Neuroscience Methods 245, pp. 182-204. DOI: $10.1016 / j \cdot$ jneumeth.2015.01.029.

Haider, Bilal, David P.A. Schulz, Michael Häusser, and Matteo Carandini (2016). "Millisecond Coupling of Local Field Potentials to Synaptic Currents in the Awake Visual Cortex". In: Neuron 90.1, pp. 35-42. DOI: 10.1016/j . neuron . 2016.02 .034$.

Hall, Thomas M., Kianoush Nazarpour, and Andrew Jackson (2014). "Real-time estimation and biofeedback of single-neuron firing rates using local field potentials". In: Nature Communications 5.1. DOI: 10.1038/ncomms6462.

Hassabis, Demis, Dharshan Kumaran, Christopher Summerfield, and Matthew Botvinick (2017). "Neuroscience-Inspired Artificial Intelligence". In: Neuron 95.2, pp. 245-258. DOI: 10.1016/j .neuron. 2017.06.011.

Hay, Etay, Sean Hill, Felix Schürmann, Henry Markram, and Idan Segev (2011). "Models of Neocortical Layer 5b Pyramidal Cells Capturing a Wide Range of Dendritic and Perisomatic Active Properties". In: PLOS Computational Biology 7.7. Ed. by Lyle J. Graham, e1002107. DOI: 10.1371/journal .pcbi.1002107.

Hodgkin, A. L. and A. F. Huxley (1952). "A quantitative description of membrane current and its application to conduction and excitation in nerve". In: The Journal of Physiology 117.4, pp. 500-544. DOI: 10.1113/jphysiol . 1952. sp004764. 
Hubel, David H. (1957). “Tungsten Microelectrode for Recording from Single Units”. In: Science 125.3247, pp. 549550. DOI: $10.1126 /$ science.125.3247.549.

Hämäläinen, Matti, Riitta Hari, Risto J. Ilmoniemi, Jukka Knuutila, and Olli V. Lounasmaa (1993). "Magnetoencephalography - theory, instrumentation, and applications to noninvasive studies of the working human brain". In: Reviews of Modern Physics 65.2, pp. 413-497. DOI: 10.1103/revmodphys .65.413.

Jirsa, V.K., T. Proix, D. Perdikis, M.M. Woodman, H. Wang, J. Gonzalez-Martinez, C. Bernard, C. Bénar, M. Guye, P. Chauvel, and F. Bartolomei (2017). "The Virtual Epileptic Patient: Individualized whole-brain models of epilepsy spread". In: NeuroImage 145, pp. 377-388. DOI: 10.1016/j .neuroimage .2016.04.049.

Koch, Christof (1984). "Cable theory in neurons with active, linearized membranes". In: Biological Cybernetics 50.1, pp. 15-33. DOI: 10.1007/BF00317936.

Koch, Christof and Allan Jones (2016). "Big Science, Team Science, and Open Science for Neuroscience". In: Neuron 92.3, pp. 612-616. DOI: 10.1016/j.neuron. 2016.10.019.

Lamprecht, Raphael and Joseph LeDoux (2004). "Structural plasticity and memory". In: Nature Reviews Neuroscience 5.1, pp. 45-54. DOI: $10.1038 / \mathrm{nrn} 1301$.

Larkum, M. E., J. J. Zhu, and B. Sakmann (2001). "Dendritic mechanisms underlying the coupling of the dendritic with the axonal action potential initiation zone of adult rat layer 5 pyramidal neurons". In: The Journal of Physiology 533.2, pp. 447-466. DOI: $10.1111 /$ j.1469-7793.2001.0447a.x.

Leon, Paula Sanz, Stuart A. Knock, M. Marmaduke Woodman, Lia Domide, Jochen Mersmann, Anthony R. McIntosh, and Viktor Jirsa (June 2013). "The Virtual Brain: a simulator of primate brain network dynamics". In: Frontiers in Neuroinformatics 7. DOI: 10.3389/fninf .2013.00010.

Lindén, Henrik, Espen Hagen, Szymon Łęski, Eivind S. Norheim, Klas H. Pettersen, and Gaute T. Einevoll (2014). "LFPy: a tool for biophysical simulation of extracellular potentials generated by detailed model neurons". In: Frontiers in Neuroinformatics 7.41, pp. 1-15. DOI: 10.3389/fninf.2013.00041.

Lindén, Henrik, Klas H. Pettersen, and Gaute T. Einevoll (2010). "Intrinsic dendritic filtering gives low-pass power spectra of local field potentials". In: Journal of Computational Neuroscience 29.3, pp. 423-444. DOI: 10.1007/ s10827-010-0245-4.

Logothetis, Nikos K, Christoph Kayser, and Axel Oeltermann (2007). "In vivo measurement of cortical impedance spectrum in monkeys: implications for signal propagation." In: Neuron 55.5, pp. 809-23. DOI: 10.1016/j . neuron . 2007.07 .027$.

Magee, Jeffrey C. and Christine Grienberger (2020). "Synaptic Plasticity Forms and Functions". In: Annual Review of Neuroscience 43.1. PMID: 32075520, pp. 95-117. DOI: 10.1146/annurev-neuro-090919-022842.

Magnusson, Steinn Hauser (2021). "Evaluating Approximate Methods for Computing Extracellular Potentials from Neuronal Networks". MA thesis. Department of Physics, University of Oslo. URL: http://urn. nb. no/URN : NBN: no-89738.

Major, Guy, Matthew E. Larkum, and Jackie Schiller (2013). "Active Properties of Neocortical Pyramidal Neuron Dendrites". In: Annual Review of Neuroscience 36.1, pp. 1-24. DOI: 10.1146/annurev-neuro-062111-150343.

Markram, Henry, Eilif Muller, Srikanth Ramaswamy, Michael W. Reimann, Marwan Abdellah, Carlos Aguado Sanchez, Anastasia Ailamaki, Lidia Alonso-Nanclares, Nicolas Antille, Selim Arsever, et al. (2015). "Reconstruction and Simulation of Neocortical Microcircuitry". In: Cell 163.2, pp. 456-492. DOI: 10.1016/j .cell.2015.09.029.

Martínez-Cañada, Pablo, Torbjørn V. Ness, Gaute T. Einevoll, Tommaso Fellin, and Stefano Panzeri (2021). "Computation of the electroencephalogram (EEG) from network models of point neurons". In: PLOS Computational Biology 17.4. Ed. by Daniele Marinazzo, e1008893. DOI: 10.1371 /journal .pcbi.1008893.

Mazzoni, Alberto, Henrik Lindén, Hermann Cuntz, Anders Lansner, Stefano Panzeri, and Gaute T. Einevoll (2015). "Computing the Local Field Potential (LFP) from Integrate-and-Fire Network Models". In: PLOS Computational Biology 11.12. Ed. by Arnd Roth, e1004584. DOI: 10.1371/journal.pcbi.1004584.

Miceli, Stéphanie, Torbjørn V. Ness, Gaute T. Einevoll, and Dirk Schubert (2017). "Impedance Spectrum in Cortical Tissue: Implications for Propagation of LFP Signals on the Microscopic Level". In: eNeuro 4.1, ENEURO.029116.2016. DOI: 10.1523/eneuro.0291-16.2016.

Mochizuki, Y., T. Onaga, H. Shimazaki, T. Shimokawa, Y. Tsubo, R. Kimura, A. Saiki, Y. Sakai, Y. Isomura, S. Fujisawa, et al. (2016). "Similarity in Neuronal Firing Regimes across Mammalian Species". In: Journal of Neuroscience 36.21, pp. 5736-5747. DOI: 10.1523/jneurosci.0230-16.2016.

Næss, Solveig, Geir Halnes, Espen Hagen, Donald J. Hagler, Anders M. Dale, Gaute T. Einevoll, and Torbjørn V. Ness (2021). "Biophysically detailed forward modeling of the neural origin of EEG and MEG signals". In: NeuroImage 225.117467, p. 117467. ISSN: 1053-8119. DOI: 10.1016/j. neuroimage.2020.117467.

Nagendra Babu, Pooja, Charl Linssen, Jochen Martin Eppler, Tobias Schulte to Brinke, Abolfazl Ziaeemehr, Tanguy Fardet, Younes Bouhadjar, Renato Duarte, Bernhard Rumpe, and Abigail Morrison (2021). NESTML 4.0. DOI: 10.5281/ZENODO . 4740083 .

Nauhaus, Ian, Laura Busse, Matteo Carandini, and Dario L Ringach (2009). "Stimulus contrast modulates functional connectivity in visual cortex". In: Nature Neuroscience 12.1, pp. 70-76. DOI: 10.1038/nn.2232. 
Ness, Torbjørn V, Michiel W H Remme, and Gaute T Einevoll (2016). "Active subthreshold dendritic conductances shape the local field potential". In: Journal of Physiology 594.13, pp. 3809-3825.

Ness, Torbjørn V., Michiel W.H. Remme, and Gaute T. Einevoll (2018). "h-Type Membrane Current Shapes the Local Field Potential from Populations of Pyramidal Neurons". In: The Journal of Neuroscience 38.26, pp. 6011-6024. DOI: $10.1523 /$ jneurosci.3278-17.2018.

Nicholson, C. and J. A. Freeman (1975). "Theory of current source-density analysis and determination of conductivity tensor for anuran cerebellum". In: Journal of Neurophysiology 38.2, pp. 356-368. DOI: 10.1152/jn. 1975.38.2. 356.

Nunez, Paul L and Ramesh Srinivasan (2006). Electric fields of the brain: The neurophysics of EEG. New York: Oxford University Press, USA.

Pettersen, Klas H., Anna Devor, Istvan Ulbert, Anders M. Dale, and Gaute T. Einevoll (2006). "Current-source density estimation based on inversion of electrostatic forward solution: Effects of finite extent of neuronal activity and conductivity discontinuities”. In: Journal of Neuroscience Methods 154.1-2, pp. 116-133. DOI: 10.1016/j . jneumeth.2005.12.005.

Pettersen, Klas H. and Gaute T. Einevoll (2008). "Amplitude Variability and Extracellular Low-Pass Filtering of Neuronal Spikes". In: Biophysical Journal 94.3, pp. 784-802. DOI: 10.1529/biophysj .107.111179.

Plesser, Hans E. (2018). "Reproducibility vs. Replicability: A Brief History of a Confused Terminology". In: Frontiers in Neuroinformatics 11. DOI: 10.3389/fninf .2017.00076.

Plotnikov, Dimitri, Inga Blundell, Tammo Ippen, Jochen Martin Eppler, Abigail Morrison, and Bernhard Rumpe (2016). "NESTML: a modeling language for spiking neurons". In: Modellierung 2016. Ed. by Andreas Oberweis and Ralf Reussner. Bonn: Gesellschaft für Informatik e.V., pp. 93-108.

Potjans, Tobias C. and Markus Diesmann (2014). "The Cell-Type Specific Cortical Microcircuit: Relating Structure and Activity in a Full-Scale Spiking Network Model”. In: Cerebral Cortex 24.3, pp. 785-806. DOI: 10.1093/cercor/ bhs358.

Rasch, M., N. K. Logothetis, and G. Kreiman (2009). "From Neurons to Circuits: Linear Estimation of Local Field Potentials". In: Journal of Neuroscience 29.44, pp. 13785-13796. DOI: 10.1523/JNEUROSCI . 2390-09. 2009.

Remme, Michiel W. H. and John Rinzel (2011). "Role of active dendritic conductances in subthreshold input integration". In: Journal of Computational Neuroscience 31.1, pp. 13-30. DOI: 10.1007/s10827-010-0295-7.

Renart, Alfonso, Jaime de la Rocha, Peter Bartho, Liad Hollender, Néstor Parga, Alex Reyes, and Kenneth D. Harris (2010). "The Asynchronous State in Cortical Circuits". In: Science 327.5965, pp. 587-590. DOI: 10.1126/science . 1179850.

Rotter, Stefan and Markus Diesmann (1999). "Exact digital simulation of time-invariant linear systems with applications to neuronal modeling”. In: Biological Cybernetics 81.5-6, pp. 381-402. DOI: 10.1007/s004220050570.

Rössert, Christian, Christian Pozzorini, Giuseppe Chindemi, Andrew P. Davison, Csaba Eroe, James King, Taylor H. Newton, Max Nolte, Srikanth Ramaswamy, Michael W. Reimann, et al. (2017). Automated point-neuron simplification of data-driven microcircuit models. arXiv: 1604.00087 [q-bio.NC] .

Schmidt, Maximilian, Rembrandt Bakker, Kelly Shen, Gleb Bezgin, Markus Diesmann, and Sacha Jennifer van Albada (2018). "A multi-scale layer-resolved spiking network model of resting-state dynamics in macaque visual cortical areas". In: PLOS Computational Biology 14.10. Ed. by Lyle J. Graham, e1006359. DOI: 10.1371/journal . pcbi . 1006359.

Schutter, Erik de (2010). Computational modeling methods for neuroscientists. Cambridge, Mass: MIT Press. ISBN: 9780262013277.

Schwalger, Tilo, Moritz Deger, and Wulfram Gerstner (2017). "Towards a theory of cortical columns: From spiking neurons to interacting neural populations of finite size". In: PLOS Computational Biology 13.4. Ed. by Lyle J. Graham, e1005507. DOI: 10.1371/journal.pcbi.1005507.

Senk, Johanna, Corto Carde, Espen Hagen, Torsten W. Kuhlen, Markus Diesmann, and Benjamin Weyers (2018a). "VIOLA-A Multi-Purpose and Web-Based Visualization Tool for Neuronal-Network Simulation Output". In: Frontiers in Neuroinformatics 12. DOI: 10.3389/fninf .2018.00075.

Senk, Johanna, Espen Hagen, Sacha J. van Albada, and Markus Diesmann (2018b). Reconciliation of weak pairwise spike-train correlations and highly coherent local field potentials across space. arXiv: 1805.10235 [q-bio. NC] .

Senk, Johanna, Karolína Korvasová, Jannis Schuecker, Espen Hagen, Tom Tetzlaff, Markus Diesmann, and Moritz Helias (2020). "Conditions for wave trains in spiking neural networks". In: Physical Review Research 2.2, p. 023174. DOI: 10.1103 /physrevresearch . 2.023174.

Skaar, Jan-Eirik W., Alexander J. Stasik, Espen Hagen, Torbjørn V. Ness, and Gaute T. Einevoll (2020). "Estimation of neural network model parameters from local field potentials (LFPs)". In: PLOS Computational Biology 16.3. Ed. by Arnd Roth, e1007725. DOI: 10.1371/journal.pcbi.1007725.

Stoelzel, C. R., Y. Bereshpolova, A. G. Gusev, and H. A. Swadlow (2008). "The Impact of an LGNd Impulse on the Awake Visual Cortex: Synaptic Dynamics and the Sustained/Transient Distinction". In: Journal of Neuroscience 28.19, pp. 5018-5028. DOI: 10.1523/jneurosci.4726-07.2008. 
Swadlow, Harvey A., Alexander G. Gusev, and Tatiana Bezdudnaya (2002). "Activation of a Cortical Column by a Thalamocortical Impulse". In: Journal of Neuroscience 22.17, pp. 7766-7773. ISSN: 02706474. DOI: 10.1523/ jneurosci.22-17-07766.2002.

Teleńczuk, Bartosz, Nima Dehghani, Michel Le Van Quyen, Sydney S. Cash, Eric Halgren, Nicholas G. Hatsopoulos, and Alain Destexhe (2017). "Local field potentials primarily reflect inhibitory neuron activity in human and monkey cortex". In: Scientific Reports 7.1. DOI: 10.1038/srep40211.

Teleńczuk, Bartosz, Maria Teleńczuk, and Alain Destexhe (2020a). "A kernel-based method to calculate local field potentials from networks of spiking neurons". In: Journal of Neuroscience Methods 344, p. 108871. DOI: 10.1016/ j.jneumeth.2020.108871.

Teleńczuk, Maria, Bartosz Teleńczuk, and Alain Destexhe (2020b). "Modelling unitary fields and the single-neuron contribution to local field potentials in the hippocampus". In: Journal of Physiology 598.18, pp. 3957-3972. ISSN: 14697793. DOI: $10.1113 / \mathrm{JP} 279452$.

Tetzlaff, Tom, Moritz Helias, Gaute T. Einevoll, and Markus Diesmann (2012). "Decorrelation of Neural-Network Activity by Inhibitory Feedback". In: PLoS Computational Biology 8.8. Ed. by Nicolas Brunel, e1002596. DOI: 10.1371/journal.pcbi.1002596.

Tomsett, Richard J., Matt Ainsworth, Alexander Thiele, Mehdi Sanayei, Xing Chen, Marc A. Gieselmann, Miles A. Whittington, Mark O. Cunningham, and Marcus Kaiser (2014). "Virtual Electrode Recording Tool for EXtracellular potentials (VERTEX): comparing multi-electrode recordings from simulated and biological mammalian cortical tissue". In: Brain Structure and Function 220.4, pp. 2333-2353. DOI: 10.1007/s00429-014-0793-x.

Welch, P. (1967). "The use of fast Fourier transform for the estimation of power spectra: A method based on time averaging over short, modified periodograms". In: IEEE Transactions on Audio and Electroacoustics 15.2, pp. 70-73. DOI: $10.1109 /$ tau. 1967.1161901.

Wilson, C. (2008). "Up and down states". In: Scholarpedia 3.6. revision \#91904, p. 1410. DOI: 10 . 4249 / scholarpedia.1410.

Wybo, Willem A. M., Daniele Boccalini, Benjamin Torben-Nielsen, and Marc-Oliver Gewaltig (2015). "A Sparse Reformulation of the Green's Function Formalism Allows Efficient Simulations of Morphological Neuron Models". In: Neural Computation 27.12, pp. 2587-2622. DOI: 10.1162/neco_a_00788. 